TRANSACTIONS OF THE

AMERICAN MATHEMATICAL SOCIETY

Volume 358, Number 12, December 2006, Pages 5587-5629

S 0002-9947(06)03923-7

Article electronically published on July 24, 2006

\title{
ASYMPTOTIC COMPACTNESS AND ABSORBING SETS FOR 2D STOCHASTIC NAVIER-STOKES EQUATIONS ON SOME UNBOUNDED DOMAINS
}

\author{
ZDZISŁAW BRZEŹNIAK AND YUHONG LI
}

\begin{abstract}
We introduce a notion of an asymptotically compact (AC) random dynamical system (RDS). We prove that for an AC RDS the $\Omega$-limit set $\Omega_{B}(\omega)$ of any bounded set $B$ is nonempty, compact, strictly invariant and attracts the set $B$. We establish that the 2D Navier Stokes Equations (NSEs) in a domain satisfying the Poincaré inequality perturbed by an additive irregular noise generate an AC RDS in the energy space $\mathrm{H}$. As a consequence we deduce existence of an invariant measure for such NSEs. Our study generalizes on the one hand the earlier results by Flandoli-Crauel (1994) and Schmalfuss (1992) obtained in the case of bounded domains and regular noise, and on the other hand the results by Rosa (1998) for the deterministic NSEs.
\end{abstract}

\section{INTRODUCTION}

The study of the asymptotic behavior of dynamical systems is one of the most important problems in mathematical physics. In the theory of deterministic infinitedimensional dynamical systems, the notion of an attractor occupies a central position (see [41]). Generalization of this theory to the stochastic case is now a welldeveloped branch in the theory of random dynamical systems. Brzeźniak, Capiński and Flandoli [8] first developed such a notion for random PDEs. Later Crauel and Flandoli [18 introduced the corresponding generalization of the attractor to stochastic PDEs. The theory of random attractors then turned out to be very useful for the study of the long-time behavior of infinite-dimensional dynamical systems. In the above-mentioned paper [18] the authors proved the existence of a global attractor for 2D stochastic Navier-Stokes equations in a bounded domain with sufficiently regular noise. However, their method does not work for either unbounded domains or for irregular noise. This is because it relies heavily on the compactness of the Sobolev embeddings (known to be no longer compact for unbounded domains). The same difficulty was encountered in the deterministic case. To overcome it Abergel [1] used weighted spaces, while Ghidaglia 27] and Rosa 37 used energy equation. But as far as we are aware no work has been done in the stochastic case. In this paper we will continue the line of research introduced by Rosa [37] and Ladyzhenskaya 33.

Received by the editors June 6, 2004 and, in revised form, December 8, 2004.

2000 Mathematics Subject Classification. Primary 60H15, 35R60; Secondary 37H10, 34F05.

Key words and phrases. Stochastic Navier-Stokes equations, unbounded domains, cylindrical white noise, asymptotic compactness, random dynamic systems, absorbing sets.

(C)2006 American Mathematical Society Reverts to public domain 28 years from publication 
Analogously to [18, we are working in the framework of a general Random Dynamical System (RDS) on a separable Banach space H. Motivated by [33] and 37. we introduce a concept of an asymptotically compact (AC) RDS and prove that in an AC RDS with every bounded set $B \subset \mathrm{H}$ we can associate a nonempty, closed and absorbing random set $\Omega_{B}$. Invariance of that set with respect to our RDS implies existence of invariant measures which in the case of stochastic NSE in 2D unbounded domains has been until now an open question. The only papers in which a similar problem was investigated (but for equations of Ginzburg-Landau type) are 22 and [38. However, the question of the uniqueness of invariant measures remains an open question. We postpone the study of the existence of global attractors for asymptotically compact RDS until a later publication. In order to show that the RDS generated by the 2D stochastic Navier-Stokes equations in 2D domains (possibly unbounded) satisfying the so-called Poincaré inequality is asymptotically compact, we exploit the energy equality. Other examples of asymptotically compact RDS will be studied elsewhere by the second-named author. Let us repeat that our approach allows us to relax assumptions on the noise, so even if the domain is bounded, our results are new as compared with [18] or [39].

As we have explained earlier, the main motivation for the research reported here was to study the long-time behaviour of stochastic parabolic equations (in the case of 2D Navier Stokes equations) in unbounded domains. We would like to point out another advantage of our approach. Since our results no longer depend on the compactness of the embedding $\mathrm{V} \hookrightarrow \mathrm{H}$ (we use the notations introduced in section 4), we do not require our initial value problem to be well posed in the space $\mathrm{V}$ and consequently, in the bounded domain case, comparing with [18 we allow our driving noise to be space-wise much rougher (thought by some to be more physical). For example, the RKHS associated to the noise can be equal to any subspace of the Sobolev space $H^{\alpha, 2}\left(D, \mathbb{R}^{2}\right)$ with any $\alpha>0$ if $D$ is $2 \mathrm{D}$ torus and with $\alpha>1 / 2$ in a general case. However, we are unable to treat the case $\alpha=0$. The existence of solutions (but not pathwise) in certain negative order Sobolev spaces is a subject of a very interesting paper by Da Prato and Debusche [19, where however only the case of $D$ being a torus is studied (which is an essential assumption).

This paper is organized as follows. In section 2, we recall the mathematical setting of the problem and introduce the concept of asymptotically compact RDS. In section 3 , we prove that for an asymptotically compact $\operatorname{RDS} \varphi$, the $\Omega$-limit set of any bounded subset $B$ is nonempty, compact, $\vartheta$-forward invariant, and attracts $B$. Furthermore, we prove the existence of an invariant measure for an asymptotically compact Markov RDS. In section 4, using the classical Galerkin approximation method and some compactness theorems, we prove the existence of the stochastic flow (and hence of RDS) associated with 2D stochastic Navier-Stokes equations in possibly unbounded Poincaré domains (i.e. satisfying the Poincaré inequality). In section 6 we construct RDS corresponding to the stochastic NSEs we study in this paper. This result seems to be new even in the bounded domain case. In section 7 we use the energy inequalities to prove the continuity of the corresponding RDS in the weak topologies. This result is the main technical difference between our approach and the earlier approaches, and it allows us to use the weak compactness of the unit ball in the Hilbert space $\mathrm{H}$ as a substitute for the compactness of the embedding $\mathrm{V} \hookrightarrow \mathrm{H}$. In section 8 we prove that the just-constructed RDS is asymptotically compact. Finally, in section 9 we prove the existence of an invariant 
measure for the 2D stochastic Navier-Stokes equations perturbed by an additive noise.

\section{Notation AND PRELIMINARIES}

Here we will introduce only the basic notions needed in this paper. A comprehensive presentation of the theory of random dynamical systems can be found in the recent monograph by Arnold [2].

A measurable dynamical system (DS) is a triple

$$
\mathfrak{T}=(\Omega, \mathcal{F}, \vartheta),
$$

where $(\Omega, \mathcal{F})$ is a measurable space and $\vartheta: \mathbb{R} \times \Omega \ni(t, \omega) \mapsto \vartheta_{t} \omega \in \Omega$ is a measurable map such that for all $t, s \in \mathbb{R}, \vartheta_{t+s}=\vartheta_{t} \circ \vartheta_{s}$. A metric DS is a quadruple

$$
\mathfrak{T}=(\Omega, \mathcal{F}, \mathbb{P}, \vartheta),
$$

where $(\Omega, \mathcal{F}, \mathbb{P})$ is a probability space and $\mathfrak{T}^{\prime}:=(\Omega, \mathcal{F}, \vartheta)$ is a measurable DS such that for each $t \in \mathbb{R}, \vartheta_{t}: \Omega \rightarrow \Omega$ preserves $\mathbb{P}$.

Suppose also that $(\mathrm{X}, d)$ is a Polish space (i.e. complete separable metric space) and $\mathcal{B}$ is its Borel $\sigma$-field. Let $\mathbb{R}^{+}=[0, \infty)$.

Definition 2.1. Given a metric DS $\mathfrak{T}$ and a Polish space $\mathrm{X}$, a map $\varphi: \mathbb{R}^{+} \times \Omega \times \mathrm{X} \ni$ $(t, \omega, x) \mapsto \varphi(t, \omega) x \in \mathrm{X}$ is called a measurable random dynamical system (RDS) (on X over $\mathfrak{T}$ ) iff

(i) $\varphi$ is $\left(\mathcal{B}\left(\mathbb{R}^{+}\right) \otimes \mathcal{F} \otimes \mathcal{B}, \mathcal{B}\right)$-measurable;

(ii) $\varphi(t+s, \omega)=\varphi\left(t, \vartheta_{s} \omega\right) \circ \varphi(s, \omega)$ for all $s, t \in \mathbb{R}^{+}$and $\varphi(0, \omega)=i d$, for all $\omega \in \Omega$. (Cocycle property)

An $\operatorname{RDS} \varphi$ is said to be continuous or differentiable iff for all $(t, \omega) \in \mathbb{R}^{\times} \Omega$, $\varphi(t, \cdot, \omega): \mathrm{X} \rightarrow \mathrm{X}$ is continuous or differentiable, respectively. Similarly, an $\mathrm{RDS} \varphi$ is said to be time continuous iff for all $\omega \in \Omega$ and for all $x \in \mathrm{X}, \varphi(\cdot, x, \omega): \mathbb{R}^{+} \rightarrow \mathrm{X}$ is continuous.

Remark 2.2. (i) Arnold in [2] considers an $\operatorname{RDS} \varphi: \mathbb{T} \times \Omega \times \mathrm{X} \rightarrow \mathrm{X}$ over a measurable dynamical system $\left(\vartheta_{t}\right)_{t \in \mathbb{T}}($ on a fixed probability space $(\Omega, \mathcal{F}, \mathbb{P}))$ with $\mathbb{T}=\mathbb{R}, \mathbb{Z}, \mathbb{R}^{+}$ or $\mathbb{Z}^{+}$. In his case the set $\mathbb{T}$ is the same for both $\varphi$ and $\vartheta$. We only consider the continuous time case, but the set $\mathbb{T}$ for $\varphi$ is equal to $\mathbb{R}^{+}$while for $\vartheta$ it is equal to $\mathbb{R}$.

(ii) Because our interest lies in nonlocally compact metric spaces (in particular, in infinite-dimensional Banach spaces), our definition of continuous RDS is different from that in 2]; see Definition 1.1.2 therein.

For two nonempty sets $A, B \subset X$, we put

$$
d(A, B)=\sup _{x \in A} d(x, B) \quad \text { and } \quad \rho(A, B)=\max \{d(A, B), d(B, A)\} .
$$

The latter is called the Hausdorff metric (see Castaing and Valadier [15]). In fact, $\rho$ restricted to the family $\mathcal{C}$ of all nonempty closed subsets of $\mathrm{X}$ is a metric; see [15]. From now on, let $\mathcal{X}$ be the $\sigma$-field on $\mathcal{C}$ generated by open sets with respect to the Hausdorff metric $\rho$; e.g. [8], [15] or Crauel [16].

Definition 2.3. Let $(\Omega, \mathcal{F})$ be a measurable space and let $(\mathrm{X}, d)$ be a Polish space. A set-valued map $C: \Omega \rightarrow \mathcal{C}$ is said to be measurable iff $C$ is $(\mathcal{F}, \mathcal{X})$-measurable. Such a map $C$ is often called a closed random set. 
Definition 2.4. Let $\varphi: \mathbb{R}^{+} \times \Omega \times \mathrm{X} \ni(t, \omega, x) \mapsto \varphi(t, \omega) x \in \mathrm{X}$ be a measurable $\mathrm{RDS}$ on a Polish space $(\mathrm{X}, d)$ over a metric DS $\mathfrak{T}$. A closed random set $B$ is called $\varphi$-forward invariant iff for all $\omega \in \Omega$,

$$
\varphi(t, \omega) B(\omega) \subseteq B\left(\vartheta_{t} \omega\right) \quad \text { for all } t>0 .
$$

A closed random set $B$ is said to be strictly $\varphi$-forward invariant iff for all $\omega \in \Omega$,

$$
\varphi(t, \omega) B(\omega)=B\left(\vartheta_{t} \omega\right) \quad \text { for all } t>0 .
$$

Remark 2.5. By substituting $\vartheta_{-t} \omega$ for $\omega$, we get the following equivalent version of Definition 2.4.

A closed random set $B$ is $\varphi$-forward invariant, resp. strictly $\varphi$-forward invariant, iff for all $\omega \in \Omega$,

$$
\varphi\left(t, \vartheta_{-t} \omega\right) B\left(\vartheta_{-t} \omega\right) \subseteq B(\omega) \quad \text { for all } \quad t>0,
$$

or respectively,

$$
\varphi\left(t, \vartheta_{-t} \omega\right) B\left(\vartheta_{-t} \omega\right)=B(\omega) \quad \text { for all } \quad t>0 .
$$

Definition 2.6. For a given closed random set $B$, the $\Omega$-limit set of $B$ is defined to be the set

$$
\Omega(B, \omega)=\Omega_{B}(\omega)=\bigcap_{T \geq 0} \overline{\bigcup_{t \geq T} \varphi\left(t, \vartheta_{-t} \omega\right) B\left(\vartheta_{-t} \omega\right)} .
$$

Remark 2.7. (i) A priori $\Omega(B, \omega)$ can be an empty set.

(ii) We have the following equivalent version of Definition 2.6.

$$
\Omega_{B}(\omega)=\left\{y: \exists t_{n} \rightarrow \infty,\left\{x_{n}\right\} \subset B\left(\vartheta_{-t_{n}} \omega\right), \lim _{n \rightarrow \infty} \varphi\left(t_{n}, \vartheta_{-t_{n}} \omega\right) x_{n}=y\right\} .
$$

(iii) Since $\overline{\bigcup_{t \geq T} \varphi\left(t, \vartheta_{-t} \omega\right) B\left(\vartheta_{-t} \omega\right)}$ is closed, $\Omega_{B}(\omega)$ is closed as well.

Definition 2.8. A closed random set $K(\omega)$ is said to

(a) attract,

(b) absorb,

(c) $\rho$-attract

another closed random set $B(\omega)$ iff for all $\omega \in \Omega$, respectively,

(a) $\lim _{t \rightarrow \infty} d\left(\varphi\left(t, \vartheta_{-t} \omega\right) B\left(\vartheta_{-t} \omega\right), K(\omega)\right)=0$;

(b) there exists a time $t_{B}(\omega)$ such that

$$
\varphi\left(t, \vartheta_{-t} \omega\right) B\left(\vartheta_{-t} \omega\right) \subset K(\omega) \quad \text { for all } t \geq t_{B}(\omega) .
$$

$$
\lim _{t \rightarrow \infty} \rho\left(\varphi\left(t, \vartheta_{-t} \omega\right) B\left(\vartheta_{-t} \omega\right), K(\omega)\right)=0 .
$$

The smallest $t_{B}(\omega) \geq 0$ for which (2.4) holds is called the absorbtion time (of $B(\omega)$ by $K(\omega))$.

Remark 2.9. (i) Obviously, if a closed random set $K$ absorbs a closed random set $B$, then $K \rho$-attracts $B$, and if $K \rho$-attracts $B$, then $K$ attracts $B$.

(ii) Note that $\varphi\left(t, \vartheta_{-t} \omega\right) x$ can be looked at as the position of the trajectory at time 0 , which was in $x$ at time $-t$.

(iii) By replacing $\omega$ by $\vartheta_{-s} \omega$ and $t$ by $t-s$, we get the following equivalent form of part (b) of Definition 2.8. A closed random set $K$ is said to absorb another closed random set $B$ iff for all $\omega \in \Omega$, there exists a random time $\tau_{B}$ such that for $t \geq s+\tau_{B}$

$$
\varphi\left(t-s, \vartheta_{-t} \omega\right) B\left(\vartheta_{-t} \omega\right) \subset K\left(\vartheta_{-s} \omega\right)
$$


The following definition is new in the framework of RDS. It is motivated by works of Ladyzhenskaya [33, Ghidaglia [27] and Rosa [37.

Definition 2.10. We say that an $\operatorname{RDS} \varphi$ defined on a separable Banach space $\mathrm{X}$ is asymptotically compact iff for all $\omega \in \Omega$, for any sequence $\left(t_{n}\right)$ such that $t_{n} \rightarrow \infty$ and any bounded X-valued sequence $\left\{x_{n}\right\}_{n}$, the set $\left\{\varphi\left(t_{n}, \vartheta_{-t_{n}} \omega\right) x_{n}: n \in \mathbb{N}\right\}$ is relatively compact in $\mathrm{X}$.

Remark 2.11. (i) Crauel, Debussche and Flandoli in [17] have proposed a different definition of asymptotic compactness for stochastic evolutional systems. However, their definition appears to be different from ours, and it was not applied to the SPDEs in unbounded domains. It seems to us that the approach from [17 is not applicable to stochastic Navier Stokes equations in unbounded domains.

(ii) The reason we consider a Banach space in Definition 2.10, instead of a general Polish space, is subject to the necessity of considering a proper subfamily of the family of all subsets of X. Obviously, one cannot expect the set $\left\{\varphi\left(t_{n}, \vartheta_{-t_{n}} \omega\right) x_{n}\right.$ : $n \in \mathbb{N}\}$ to be relatively compact in $\mathrm{X}$ for any $\mathrm{X}$-valued sequence $\left\{x_{n}\right\}_{n}$. One can easily generalize the above definition to a Polish space in the following way. Suppose that $\mathrm{X}$ is endowed with a family $\mathcal{G}$ of nonempty subsets of X. We say that the RDS $\varphi$ on $X$ over $\vartheta$ is $\mathcal{G}$-asymptotically compact iff for any $B \in \mathcal{G}$, any sequence $\left(t_{n}\right): t_{n} \rightarrow \infty$ and $B$-valued sequence $\left\{x_{n}\right\}_{n}$, the set $\left\{\varphi\left(t_{n}, \vartheta_{-t_{n}} \omega\right) x_{n}: n \in \mathbb{N}\right\}$ is relatively compact in $\mathrm{X}$.

\section{Properties of the $\Omega$-Limit Set}

Throughout this section we will assume that $\mathfrak{T}=(\Omega, \mathcal{F}, \mathbb{P}, \vartheta)$ is a metric DS, X is a separable Banach space, and $\varphi$ is a continuous, asymptotically compact RDS on $\mathrm{X}$ (over $\mathfrak{T}$ ). We begin our discussion with the following fundamental property.

Proposition 3.1. If $B \subset \mathrm{X}$ is a bounded deterministic set, then for all $\omega \in \Omega$, $\Omega_{B}(\omega)$ is a nonempty set.

Proof. Since $B$ is bounded, by the asymptotic compactness of $\varphi$, we may assume that for all $\omega \in \Omega$, there exists a $B$-valued sequence $\left\{x_{n}\right\}_{n}$ and an $\mathbb{R}^{+}$-valued sequence $\left(t_{n}\right)_{n}$, and an element $y \in \mathrm{X}$ such that

$$
\lim _{n \rightarrow \infty} \varphi\left(t_{n}, \vartheta_{-t_{n}} \omega\right) x_{t_{n}}=y \quad \text { in X. }
$$

Therefore, by Remark 2.7(i), $y \in \Omega_{B}(\omega)$, which proves that $\Omega_{B}(\omega) \neq \emptyset$.

Theorem 3.2. If $B \subset \mathrm{X}$ is a bounded deterministic set, then for all $\omega \in \Omega, \Omega_{B}(\omega)$ attracts $B$.

Proof. Suppose that there is an $\omega \in \Omega$ such that $\Omega_{B}(\omega)$ does not attract $B$. Hence we can find a number $\delta>0$, a sequence $\left(t_{n}\right)_{n}: t_{n} \rightarrow \infty$ and a $B$-valued sequence $\left\{b_{n}\right\}_{n}$, such that

$$
d\left(\varphi\left(t_{n}, \vartheta_{-t_{n}} \omega\right) b_{n}, \Omega_{B}(\omega)\right) \geq \delta \quad \text { for all } n \in \mathbb{N} .
$$

Since $\varphi$ is asymptotically compact, $\left\{\varphi\left(t_{n}, \vartheta_{-t_{n}} \omega\right) b_{n}: n \in \mathbb{N}\right\}$ is relatively compact in $\mathrm{X}$. Therefore there exists a subsequence $\left(n^{\prime}\right) \subset(n)$ and $y \in \mathrm{X}$, such that

$$
\lim _{n^{\prime} \rightarrow \infty} \varphi\left(t_{n^{\prime}}, \vartheta_{-t_{n^{\prime}}} \omega\right) b_{n^{\prime}}=y .
$$

By Remark 2.7(i), $y \in \Omega_{B}(\omega)$. On the other hand, (3.1) and (3.2) together imply that $d\left(y, \Omega_{B}(\omega)\right) \geq \delta>0$. This contradiction completes the proof. 
Theorem 3.3. If $B \subset \mathrm{X}$ is a bounded deterministic set, then for all $\omega \in \Omega, \Omega_{B}(\omega)$ is strictly $\varphi$-forward invariant.

Proof. Let $\omega \in \Omega$. By Remark 2.7, if $y \in \Omega_{B}(\omega)$, we can find a sequence $\left(t_{n}\right)$ : $t_{n} \rightarrow \infty$ and a $B$-valued sequence $\left\{x_{n}\right\}_{n}$, such that $y=\lim _{n \rightarrow \infty} \varphi\left(t_{n}, \vartheta_{-t_{n}} \omega\right) x_{n}$.

Let $t>0$. By the cocycle property we have

$$
\varphi\left(t_{n}+t, \vartheta_{-t_{n}} \omega\right)=\varphi\left(t, \vartheta_{t_{n}} \vartheta_{-t_{n}} \omega\right) \varphi\left(t_{n}, \vartheta_{-t_{n}} \omega\right)=\varphi(t, \omega) \varphi\left(t_{n}, \vartheta_{-t_{n}} \omega\right) .
$$

Moreover, since by our assumption $\varphi(t, \omega): \mathrm{X} \rightarrow \mathrm{X}$ is continuous, we infer that

$$
\begin{aligned}
\varphi(t, \omega) y & =\varphi(t, \omega) \lim _{n \rightarrow \infty} \varphi\left(t_{n}, \vartheta_{-t_{n}} \omega\right) x_{n}=\lim _{n \rightarrow \infty} \varphi(t, \omega) \varphi\left(t_{n}, \vartheta_{-t_{n}} \omega\right) x_{n} \\
& =\lim _{n \rightarrow \infty} \varphi\left(t_{n}+t, \vartheta_{-t_{n}} \omega\right) x_{n}=\lim _{n \rightarrow \infty} \varphi\left(t_{n}+t, \vartheta_{-t_{n}-t} \vartheta_{t} \omega\right) x_{n} \\
& =\lim _{n \rightarrow \infty} \varphi\left(s_{n}, \vartheta_{-s_{n}} \vartheta_{t} \omega\right) x_{n},
\end{aligned}
$$

where $s_{n}=t+t_{n}$. Since $\lim _{n \rightarrow \infty} s_{n}=\infty$, we infer that $\lim _{n \rightarrow \infty} \varphi\left(s_{n}, \vartheta_{-s_{n}} \vartheta_{t} \omega\right) x_{n}$ exists and belongs to $\Omega_{B}\left(\vartheta_{t} \omega\right)$. This proves that $\varphi(t, \omega) \Omega_{B}(\omega) \subset \Omega_{B}\left(\vartheta_{t} \omega\right)$.

Conversely, suppose $y \in \Omega_{B}\left(\vartheta_{t} \omega\right)$ for some $t>0$. Hence, by Remark 2.7(i), we can find a sequence $\left(t_{n}\right)_{n}: t_{n} \rightarrow \infty$ and a $B$-valued sequence $\left\{x_{n}\right\}$, such that

$$
y=\lim _{n \rightarrow \infty} \varphi\left(t_{n}, \vartheta_{-t_{n}} \vartheta_{t} \omega\right) x_{n} .
$$

Since $t_{n}-t \rightarrow \infty$, in view of the asymptotic compactness of the $\operatorname{RDS} \varphi$, the set $\left\{\varphi\left(t_{n}-t, \vartheta_{-\left(t_{n}-t\right)} \omega\right) x_{n}: n \in \mathbb{N}\right\}$ is relatively compact in X. Hence there exists a subsequence $\left(t_{n^{\prime}}\right)_{n^{\prime}} \subset\left(t_{n}\right)_{n}$ and an element $z \in \mathrm{X}$ such that

$$
\varphi\left(t_{n^{\prime}}-t, \vartheta_{-\left(t_{n^{\prime}}-t\right)} \omega\right) x_{n^{\prime}} \rightarrow z .
$$

Therefore $z \in \Omega_{B}(\omega)$. On the other hand $y=\varphi(t, \omega) z$. Indeed, by the continuity of $\varphi(t, \omega): \mathrm{X} \rightarrow \mathrm{X}$ we have

$$
y=\lim _{n^{\prime} \rightarrow \infty} \varphi\left(t_{n^{\prime}}, \vartheta_{-t_{n^{\prime}}} \vartheta_{t} \omega\right) x_{n^{\prime}}=\varphi(t, \omega) \lim _{n^{\prime} \rightarrow \infty} \varphi\left(t_{n^{\prime}}-t, \vartheta_{-t_{n^{\prime}}+t_{t}} \omega\right) x_{n^{\prime}}=\varphi(t, \omega) z .
$$

In particular, $y \in \varphi(t, \omega) \Omega_{B}(\omega)$, which proves that $\Omega_{B}\left(\vartheta_{t} \omega\right) \subset \varphi(t, \omega) \Omega_{B}(\omega)$.

Theorem 3.4. If $B \subset \mathrm{X}$ is a bounded deterministic set, then for all $\omega \in \Omega, \Omega_{B}(\omega)$ is compact.

Before embarking on proving the last theorem, let us briefly discuss the existence of an invariant measure for the $\operatorname{RDS} \varphi$. We have the following definition; see also [2], Remark 1.1.8.

Definition 3.5. The skew product of a measurable DS $\mathfrak{T}$ with an $\operatorname{RDS} \varphi$ on a Polish space $\mathrm{X}$ over $\mathfrak{T}$ is the map

$$
\Theta: \mathbb{R}^{+} \times \Omega \times \mathrm{X} \ni(t, \omega, x) \mapsto(\vartheta(t, \omega), \varphi(t, \omega) x)=\Theta_{t}(\omega, x) \in \Omega \times \mathrm{X} .
$$

One can show that if $\Theta$ is the skew product of $\mathfrak{T}$ with $\varphi$, then a triple

$$
\widehat{\mathfrak{T}}:=(\Omega \times \mathrm{X}, \mathcal{F} \otimes \mathcal{B}, \Theta)
$$

is a measurable DS. Conversely, if $\mathfrak{T}$ is a measurable DS, $\vartheta: \mathbb{R}^{+} \times \Omega \times \mathrm{X} \rightarrow \mathrm{X}$ is measurable, the function $\Theta$ defined by (3.3) and the triple $\widehat{\mathfrak{T}}$ is a measurable DS, then $\varphi$ is an RDS on $\mathrm{X}$ over $\mathfrak{T}$. 
Definition 3.6. Let $\varphi$ be a given RDS over a metric DS $\mathfrak{T}$. A probability measure $\mu$ on $(\Omega \times \mathrm{X}, \mathcal{F} \otimes \mathcal{B})$ is called an invariant measure for $\varphi$ iff

(i) $\Theta_{t}$ preserves $\mu$ (i.e. $\Theta_{t}(\mu)=\mu$ ) for each $t \in \mathbb{R}^{+}$; $\Omega$.

(ii) the first marginal of $\mu$ is $\mathbb{P}$, i.e. $\pi_{\Omega}(\mu)=\mathbb{P}$, where $\pi_{\Omega}: \Omega \times \mathrm{X} \ni(\omega, x) \mapsto \omega \in$

Since by Corollary 4.4 in Crauel and Flandoli [18], if an $\operatorname{RDS} \varphi$ on a Polish space X has an invariant compact random set $K(\omega), \omega \in \Omega$, it also has an invariant probability measure, we have the following.

Corollary 3.7. A continuous and asymptotically compact $R D S \varphi$ on a separable Banach space has at least one invariant probability measure $\mu$ in the sense of Definition 3.6.

Next we define a Feller invariant measure for a Markov $\operatorname{RDS} \varphi$. If $f: \mathrm{X} \rightarrow \mathbb{R}$ is a bounded and Borel measurable function, then we put

$$
\left(P_{t} f\right)(x)=\mathbb{E} f(\varphi(t, x)), \quad t \geq 0, x \in \mathrm{X} .
$$

We easily see that $P_{t} f$ is also a bounded and Borel measurable function. Moreover, we have the following result.

Proposition 3.8. The family $\left(P_{t}\right)_{t \geq 0}$ is Feller, i.e. $P_{t} f \in C_{b}(\mathrm{X})$ if $f \in C_{b}(\mathrm{X})$. Moreover, if the RDS $\varphi$ is time continuous, then for any $f \in C_{b}(\mathrm{X}),\left(P_{t} f\right)(x) \rightarrow$ $f(x)$ as $t \searrow 0$.

Proof. For the first assertion we only need to consider the case $t>0$ (where anyway the proof is straightforward). Indeed, if $x_{n} \rightarrow x$ in X, by the continuity of $\varphi(t, \cdot, \omega)$ : $\mathrm{X} \rightarrow \mathrm{X},\left(P_{t} f\right)\left(x_{n}\right) \rightarrow\left(P_{t} f\right)(x)$ by using the Lebesgue dominated convergence theorem.

To prove the second one, we note that from the continuity of $\varphi(\cdot, x, \omega):[0, \infty) \rightarrow$ $\mathrm{X}$ it follows that for each $x \in \mathrm{X},\left(P_{t} f\right)(x) \rightarrow f(x)$ as $t \rightarrow 0$ if $x \in \mathrm{X}$.

An RDS $\varphi$ is called Markov iff the family $\left(P_{t}\right)_{t \geq 0}$ is a semigroup on $C_{b}(\mathrm{X})$, i.e. $P_{t+s}=P_{t} P_{s}$ for all $t, s \geq 0$.

Definition 3.9. A Borel probability measure $\mu$ on $\mathrm{X}$ is called an invariant measure for a a semigroup $\left(P_{t}\right)_{t \geq 0}$ of Feller operators on $C_{b}(\mathrm{X})$ iff

$$
P_{t}^{*} \mu=\mu, t \geq 0
$$

where $\left(P_{t}^{*} \mu\right)(\Gamma)=\int_{\mathrm{H}} P_{t}(x, \Gamma) \mu(d x)$ for $\Gamma \in \mathcal{B}(\mathrm{H})$ and the $P_{t}(x, \cdot)$ is the transition probability, $P_{t}(x, \Gamma)=P_{t}\left(1_{\Gamma}\right)(x), x \in B$.

A Feller invariant probability measure for a Markov $\operatorname{RDS} \varphi$ on $\mathrm{H}$ is, by definition, an invariant probability measure for the semigroup $\left(P_{t}\right)_{t \geq 0}$ defined by (3.4).

Crauel and Flandoli proved in [18] (see Corollary 4.6) that if a Markov RDS $\varphi$ on a Polish space $\mathrm{H}$ has an invariant compact random set $K(\omega), \omega \in \Omega$, then there exists a Feller invariant probability measure $\mu$ for $\varphi$. Thus we have the following result.

Corollary 3.10. If a time-continuous and continuous Markov RDS $\varphi$ is asymptotically compact, then for any bounded set $B \subset \mathrm{H}$, there exists a Feller invariant probability measure $\mu$ for $\varphi$. 
Remark 3.11. The uniqueness of an invariant Borel probability measure (and thus its independence of the set $B$ ) remains an open question. The existence of a global attractor is another open question. Both will be studied in subsequent publications.

Proof of Theorem 3.4. Suppose that $\left\{y^{n}\right\}_{n}$ is an $\Omega_{B}(\omega)$-valued sequence. In view of Remark 2.7(i), for any $n \in \mathbb{N}$, we can find sequences $\left\{t_{k}^{n}\right\}$ and $\left\{x_{k}^{n}\right\}_{k} \subset B$ such that $t_{k}^{n} \rightarrow \infty \varphi\left(t_{k}^{n}, \vartheta_{-t_{k}^{n}} \omega\right) x_{k}^{n} \rightarrow y^{n}$. Next we construct a sequence $\left\{s_{i}\right\}_{i}$ in the following way. We put $s_{1}=t_{k_{1}}^{1}$ for $k_{1} \in \mathbb{N}$ such that $d\left(\varphi\left(t_{k_{1}}^{1}, \vartheta_{-t_{k_{1}}} \omega\right) x_{k_{1}}^{1}, y^{1}\right)<2^{-1}$. If $s_{i}$ is constructed we put $s_{i+1}=t_{k_{i+1}}^{i+1}$, where $k_{i+1} \in \mathbb{N}$ is such that $t_{k_{i+1}}^{i+1}>s_{i}+1$ and $d\left(\varphi\left(t_{k_{i+1}}^{i+1}, \vartheta_{-t_{k_{i+1}}^{i+1}} \omega\right) x_{k_{i+1}}^{i+1}, y^{i+1}\right)<2^{-i-1}$. It is obvious that $s_{i} \rightarrow \infty$. Put $z_{i}=x_{k_{i}}^{i}$, $i \in \mathbb{N}$. Since $\left\{z_{i}: i \in \mathbb{N}\right\} \subset B$, by the asymptotic compactness of the $\operatorname{RDS} \varphi$, the set $\left\{\varphi\left(s_{i}, \vartheta_{-s_{i}} \omega\right) z_{i}\right\}$ is relatively compact. Hence we can find a subsequence $s_{i^{\prime}}$ and an element $z \in \mathrm{X}$, such that $\varphi\left(s_{i^{\prime}}, \vartheta_{-s_{i^{\prime}}} \omega\right) z_{i^{\prime}} \rightarrow z$. On the other hand, the definition of $s_{i}$ implies that $d\left(\varphi\left(s_{i^{\prime}}, \vartheta_{-s_{i^{\prime}}} \omega\right) z_{i^{\prime}}, y^{i^{\prime}}\right)<2^{-i^{\prime}}$. Therefore, $y^{i^{\prime}} \rightarrow z$. Since by Remark 2.7 (ii), $\Omega_{B}(\omega)$ is closed; we infer that $z \in \Omega_{B}(\omega)$. This concludes the proof of compactness of $\Omega_{B}(\omega)$.

\section{Time Dependent 2D stochastic Navier Stokes equations}

Let us consider an incompressible viscous fluid of constant density (assumed to be equal to 1 and of constant viscosity $\nu>0$ ) enclosed in a region $D \subset \mathbb{R}^{2}$ and driven by an external time-dependent force $f: \mathbb{R}^{+} \times D \rightarrow \mathbb{R}^{2}$. We denote by $u(t, x) \in \mathbb{R}^{2}$ and $p(t, x) \in \mathbb{R}$, respectively, the velocity and the pressure of the fluid at the point $x \in D$ at time $t \geq 0$. We assume that the time evolution of the velocity and pressure of the fluid is governed by the initial-boundary value problem associated with Navier-Stokes equations:

$$
\begin{cases}\frac{\partial u}{\partial t}-\nu \Delta u+(u \cdot \nabla) u+\nabla p=f & \text { in } D, \\ \operatorname{div} u=0 & \text { in } D, \\ u=0 & \text { on } \partial D, \\ u(\cdot, 0)=u_{0} & \text { in } D .\end{cases}
$$

We assume that $D \subset \mathbb{R}^{2}$ is an arbitrary (bounded or unbounded) domain with boundary $\partial D$ satisfying the cone property.

We will use the standard mathematical framework of the NSEs; see e.g. [40]. The basic functional space is the Lebesgue space $\mathbb{L}^{2}(D):=L^{2}\left(D, \mathbb{R}^{2}\right)$ with scalar product $(u, v)=\sum_{j} \int_{D}\left(u_{j}(x) v_{j}(x)\right) d x$ and norm $|\cdot|=(\cdot, \cdot)^{1 / 2}$. We will also need the Sobolev space $\mathbb{H}^{k, p}(D)=H^{k, p}\left(D, \mathbb{R}^{2}\right), k \in \mathbb{N}$, and $p \in[1, \infty)$ consisting of all $u \in L^{p}\left(D, \mathbb{R}^{2}\right)$ whose weak derivatives up to order $k$ belong to $L^{p}\left(D, \mathbb{R}^{2}\right)$ as well. $\mathbb{H}^{k, p}(D)$ is a separable Banach space with norm

$$
\|u\|_{k, p}:=\left(\sum_{|\alpha| \leq k} \int_{D}\left|D^{\alpha} u(x)\right|^{p} d x\right)^{1 / p} .
$$

Obviously $\mathbb{H}^{k, 2}(D), k \in \mathbb{N}$, is a Hilbert space with naturally defined scalar product. We will consider the weak solutions to problem (4.1), and for this we need a proper space of test functions. We take

$$
\mathcal{V}=\mathcal{V}(D):=\left\{\phi \in C_{0}^{\infty}\left(D, \mathbb{R}^{2}\right): \operatorname{div} \phi=0 \text { in } D\right\} .
$$


The closure of $\mathcal{V}$ in $\mathbb{L}^{2}(D)$, resp. in $\mathbb{H}^{1,2}(D)$, will be denoted by $\mathrm{H}$, resp. $\mathrm{V}$. The scalar products norms in those two spaces are those inherited from $\mathbb{L}^{2}(D)$, resp. $\mathbb{H}^{1,2}(D)$.

We call $D$ a Poincaré domain iff there exists $\lambda_{1}>0$ such that

$$
\int_{D} \phi^{2} d x \leq \frac{1}{\lambda_{1}} \int_{D}|\nabla \phi|^{2} d x, \quad \phi \in C_{0}^{\infty}\left(D, \mathbb{R}^{2}\right) .
$$

The inequality (4.2) is called the Poincaré inequality, and it can be shown that if $D$ is bounded is some direction, i.e. there exists a vector $b \in \mathbb{R}^{2}$ such that $\sup _{x \in D}|(x, b)|<\infty$, then $D$ is a Poincaré domain.

If $D$ is a Poincaré domain, then the original norm on $\mathrm{V}$ is equivalent to the norm $\|\cdot\|$ induced by the scalar product

$$
((u, v))=\int_{D} \sum_{j=1}^{2} \nabla u_{j} \cdot \nabla v_{j} d x=(\nabla u, \nabla v), \quad u, v \in \mathrm{V} .
$$

We define next a bilinear form $a: \mathrm{V} \times \mathrm{V} \rightarrow \mathbb{R}$ by

$$
a(u, v):=(\nabla u, \nabla v), \quad u, v \in \mathrm{V} .
$$

Since obviously the form $a$ coincides with the $((\cdot, \cdot))$ scalar product in $\mathrm{V}$, it is $\mathrm{V}$ continuous, i.e. it satisfies $|a(u, u)| \leq C\|u\|^{2}$ for some $C>0$ and all $u \in \mathrm{V}$. Hence, by the Riesz Lemma, there exists a unique linear operator $\mathcal{A}: \mathrm{V} \rightarrow \mathrm{V}^{\prime}$, where $\mathrm{V}^{\prime}$ is the dual of $\mathrm{V}$, such that $a(u, v)=\langle\mathcal{A} u, v\rangle$, for $u, v \in \mathrm{V}$. Moreover, the form $a$ is obviously $\mathrm{V}$-coercive, i.e. it satisfies $a(u, u) \geq \alpha\|u\|^{2}$ for some $\alpha>0$ and all $u \in \mathrm{V}$. Therefore, by means of the Lax-Milgram theorem (see e.g. Temam [41, Theorem II.2.1]) the operator $\mathcal{A}: \mathrm{V} \rightarrow \mathrm{V}^{\prime}$ is an isomorphism. Since $\mathrm{V}$ is densely and continuously embedded into $\mathrm{H}$ and $\mathrm{H}$ can be identified with its dual $\mathrm{H}^{\prime}$, we have the following embeddings:

$$
\mathrm{V} \subset \mathrm{H} \cong \mathrm{H}^{\prime} \subset \mathrm{V}^{\prime}
$$

Let us then recall that we say that the spaces $\mathrm{V}, \mathrm{H}$ and $\mathrm{V}^{\prime}$ form a Gelfand triple.

Next we define an unbounded linear operator $\mathrm{A}$ in $\mathrm{H}$ as follows:

$$
\begin{cases}D(\mathrm{~A}) & :=\{u \in \mathrm{V}: \mathcal{A} u \in \mathrm{H}\} \\ \mathrm{A} u & :=\mathcal{A} u, u \in D(\mathrm{~A}) .\end{cases}
$$

It is now well established that under some additional assumptions related to the regularity of the domain $D$, the space $D(\mathrm{~A})$ can be characterized in terms of Sobolev spaces. For example (see [30, where only the 3-dimensional case is studied but the result is also valid in the 3 -dimensional case), if $D \subset \mathbb{R}^{2}$ is a uniform $C^{2}$ class Poincaré domain, then with $\mathrm{P}: \mathbb{L}^{2}(D) \rightarrow \mathrm{H}$ being the orthogonal projection, we have

$$
\begin{cases}D(\mathrm{~A}) & :=\mathrm{V} \cap \mathbb{H}^{1,2}(D), \\ \mathrm{A} u & :=-\mathrm{P} \Delta u, \quad u \in D(\mathrm{~A}) .\end{cases}
$$

It is also a classical result (see e.g. Cattabriga [14] or Temam [1], p. 56) that A is a nonnegative self-adjoint operator in $\mathrm{H}$. Moreover (see p. 57 in 41$]), \mathrm{V}=D\left(\mathrm{~A}^{1 / 2}\right)$. Let us recall a result of Fujiwara-Morimoto 26] that the projection $\mathrm{P}$ extends to a bounded linear projection in the space $\mathbb{L}^{q}(D), 1<q<\infty$.

Remark 4.1. (i) Let us denote by $\mathbb{H}_{0}^{1,2}(D)$ the closure of $C_{0}^{\infty}\left(D, \mathbb{R}^{2}\right)$ in $\mathbb{H}^{1,2}(D)$. It can be shown that $\mathrm{V}$ is equal to the closure of $\mathcal{V}$ in $\mathbb{H}_{0}^{1,2}(D)$. 
(ii) The characterization of the spaces $\mathrm{H}$ and $\mathrm{V}$ given in 40 also holds true when $D$ is a Poincaré domain. Namely, if $\vec{n}$ denotes the external normal vector field to $\partial D$, then

$$
\begin{aligned}
\mathrm{H}^{\perp} & =\left\{u \in \mathbb{L}^{2}(D): u=\operatorname{grad} p, \text { for some } p \in \mathbb{L}_{\mathrm{loc}}^{2}(D)\right\}, \\
\mathrm{H} & =\left\{u \in \mathbb{L}^{2}(D): \operatorname{div} u=0,\left.u \cdot \vec{n}\right|_{\partial D}=0\right\}, \\
\mathrm{V} & =\left\{u \in \mathbb{H}_{0}^{1,2}(D): \operatorname{div} u=0\right\} .
\end{aligned}
$$

(iii) If $D$ is a bounded domain, then the operator $\mathrm{A}$ is invertible and its inverse $\mathrm{A}^{-1}$ is bounded, self-adjoint and compact in $\mathrm{H}$. Hence the spectrum of $\mathrm{A}$ consists of an infinite sequence $0<\lambda_{1} \leq \lambda_{2} \leq \ldots \leq \lambda_{m} \rightarrow \infty$ of eigenvalues listed with their multiplicity, and there exists an orthogonal basis $\left\{w_{m}\right\}_{m \geq 1}$ of $\mathrm{H}$ consisting of eigenvectors of $\mathrm{A}: \mathrm{A} w_{m}=\lambda_{m} w_{m}, m \in \mathbb{N}$.

(iv) If $D$ is a Poincaré domain, then the operator $\mathrm{A}$ is invertible, its inverse $\mathrm{A}^{-1}$ is bounded and

$$
|\mathrm{A} u|^{2} \geq \lambda_{1}(\mathrm{~A} u, u) \geq \lambda_{1}^{2}|u|^{2} \quad \text { for all } u \in D(\mathrm{~A}) .
$$

In particular, the original norm on $\mathrm{V}$ is equivalent to the norm $\|\cdot\|$ induced by the scalar product

$$
((u, v))=\int_{D} \sum_{j=1}^{2} \nabla u_{j} \cdot \nabla v_{j} d x=(\nabla u, \nabla u) .
$$

Moreover, the graph norm on $D(\mathrm{~A})$ is equivalent to the $|\mathrm{A} \cdot|$-norm and (see p. 57 in 41])

$$
\langle\mathrm{A} u, u\rangle=((u, u))=\|u\|^{2}=|\nabla u|^{2}, \quad u \in D(\mathrm{~A}) .
$$

(v) If $D$ is not a Poincaré domain, then $\|\cdot\|$ is only a seminorm on $\mathrm{V}$. The same comment applies to the seminorm $|\mathrm{A} \cdot|$ on $D(\mathrm{~A})$.

Next, we define the following fundamental trilinear form:

$$
b(u, v, w)=\int_{D} u \nabla v w d x=\sum_{i, j=1}^{2} \int_{D} u^{i}(x) D_{i} v^{j}(x) w^{j}(x) d x,
$$

whenever $u, v, w \in \mathbb{L}_{\text {loc }}^{1}(D)$ are such that the integral on the right-hand side (RHS) exists. If $u, v$ are such that the linear map $b(u, v, \cdot)$ is continuous on $\mathrm{V}$, the corresponding element of $\mathrm{V}^{\prime}$ will be denoted by $B(u, v)$. We will also denote, with a slight abuse abuse of notation, $B(u)=B(u, u)$. Note that if $u, v \in \mathrm{H}$ are such that $(u \nabla) v=\sum_{j} u_{j} D_{j} v \in \mathbb{L}^{2}(D)$, then $B(u, v)=P(u \nabla v)$.

The following are some fundamental properties of the form $b$; see e.g. [40, Lemma 1.3, p. 163, and Temam [41. There exists a constant $C>0$ such that

$$
\begin{aligned}
& b(u, v, v)=0 \quad \text { for } u \in \mathrm{V}, v \in \mathbb{H}_{0}^{1,2}(D), \\
& b(u, v, w)=-b(u, w, v) \quad \text { for } u \in \mathrm{V}, v, w \in \mathbb{H}_{0}^{1,2}(D) .
\end{aligned}
$$

$$
|b(u, v, w)| \leq C\left\{\begin{array}{l}
|u|^{1 / 2}|\nabla u|^{1 / 2}|\nabla v|^{1 / 2}|\mathrm{~A} v|^{1 / 2}|w|, \quad u \in \mathrm{V}, v \in D(\mathrm{~A}), w \in \mathrm{H} \\
|u|^{1 / 2}|\mathrm{~A} u|^{1 / 2}|\nabla v||w|^{2} \quad u \in D(\mathrm{~A}), v \in \mathrm{V}, w \in \mathrm{H} \\
|u||\nabla v||w|^{1 / 2}|\mathrm{~A} w|^{1 / 2}, \quad u \in \mathrm{H}, v \in \mathrm{V}, w \in D(\mathrm{~A}) \\
|u|^{1 / 2}|\nabla u|^{1 / 2}|\nabla v||w|^{1 / 2}|\nabla w|^{1 / 2}, \quad u, v, w \in \mathrm{V}
\end{array}\right.
$$


Also we have, from Temam [40, Lemma III.3.3, the following inequality:

$$
|v|_{\mathbb{L}^{4}(D)} \leq 2^{1 / 4}|v|_{\mathbb{L}^{2}(D)}^{1 / 2}|\nabla v|_{\mathbb{L}^{2}(D)}^{1 / 2}, \quad v \in \mathbb{H}_{0}^{1,2}(D) .
$$

By means of the Hölder inequality we can deduce the following inequality:

$$
|b(u, v, w)| \leq|u|_{\mathbb{L}^{4}(D)}|\nabla v|_{\mathbb{L}^{2}(D)}|w|_{\mathbb{L}^{4}(D)}, \quad u, v, w \in \mathbb{H}_{0}^{1,2}(D) .
$$

Hence $b$ is a bounded trilinear map from $\mathbb{L}^{4}(D) \times \mathrm{V} \times \mathbb{L}^{4}(D)$ to $\mathbb{R}$. Moreover, we have the following result which is fundamental for our purposes.

Lemma 4.2. The trilinear map $b: \mathrm{V} \times \mathrm{V} \times \mathrm{V} \rightarrow \mathbb{R}$ has a unique extension to a bounded trilinear map from $\mathbb{L}^{4}(D) \times\left(\mathbb{L}^{4}(D) \cap \mathrm{H}\right) \times \mathrm{V}$ to $\mathbb{R}$.

It follows from Lemma 4.2 that $B$ maps $\mathbb{L}^{4}(D) \cap \mathrm{H}$ (and so $\mathrm{V}$ ) into $\mathrm{V}^{\prime}$ and

$$
|B(u)|_{\mathrm{V}^{\prime}} \leq C_{1}|u|_{\mathbb{L}^{4}(D)}^{2} \leq 2^{1 / 2} C_{1}|u||\nabla u| \leq C_{2}|u|_{V}^{2}, \quad u \in \mathrm{V} .
$$

Using the above notation it is now customary to consider the following functional analytic version of problem (4.1):

$$
\left\{\begin{array}{l}
\frac{d u}{d t}+\nu \mathrm{A} u+B(u)=f(t), t \geq 0, \\
u(0)=u_{0} .
\end{array}\right.
$$

Our aim is to study the following Navier-Stokes equations in $D$, i.e. the above problem with the external force $f(t)$ being random:

$$
\left\{\begin{array}{l}
d u+\{\nu \mathrm{A} u+B(u)\} d t=f d t+d W(t), \quad t \geq 0, \\
u(0)=x
\end{array}\right.
$$

where we assume that $x \in \mathrm{H}, f \in \mathrm{V}^{\prime}$ and $W(t), t \in \mathbb{R}$, is a two-sided cylindrical Wiener process in $\mathrm{H}$ (with its Reproducing Kernel Hilbert Space (RKHS) to be specified later; see Remark 6.1) defined on some filtered probability space $A=$ $\left(\Omega, \mathcal{F},\left(\mathcal{F}_{t}\right)_{t \in \mathbb{R}}, \mathbb{P}\right)$.

Remark 4.3. (i) Let us recall the celebrated Gagliardo-Nirenberg inequalities; see e.g. 25]. Assume that $q, r \in[1, \infty]$, and $j, m \in \mathbb{Z}$ satisfy $0 \leq j<m$. Then for any $u \in C_{0}^{m}\left(\mathbb{R}^{n}\right)$,

$$
\left\|D^{j} u\right\|_{L^{p}\left(\mathbb{R}^{n}\right)} \leq C\left\|D^{m} u\right\|_{L^{r}\left(\mathbb{R}^{n}\right)}^{a}\|u\|_{L^{q}\left(\mathbb{R}^{n}\right)}^{1-a},
$$

where $\frac{1}{p}=\frac{j}{n}+a\left(\frac{1}{r}-\frac{m}{n}\right)+(1-a) \frac{1}{q}$ for all $a \in\left[\frac{j}{m}, 1\right]$ and $C$ is a constant depending only on $n, m, j, q, r, a$ with the following exception. If $m-j-\frac{n}{r}$ is a nonnegative integer, then the equality (4.17) holds only for $a \in\left[\frac{j}{m}, 1\right)$.

In particular, if $n=2, j=0, m=1$ and $r=q=2 \leq p<\infty$, then (4.17) implies the following generalization of (4.12):

$$
|v|_{\mathbb{L}^{p}\left(\mathbb{R}^{n}\right)} \leq C|v|_{\mathbb{L}^{2}\left(\mathbb{R}^{n}\right)}^{1-\frac{2}{p}}|\nabla v|_{\mathbb{L}^{2}\left(\mathbb{R}^{n}\right)}^{\frac{2}{2}}, \quad v \in H_{0}^{1,2}\left(\mathbb{R}^{n}\right) .
$$

(ii) If $z \in L^{4}\left([0, T] ; \mathbb{L}^{4}(D)\right)$, then $B(z) \in L^{2}\left(0, T ; \mathrm{V}^{\prime}\right)$. Indeed, by (4.14) we have $\int_{0}^{T}|B(z(t))|_{\mathrm{V}^{\prime}}^{2} d t \leq C_{1}^{2} \int_{0}^{T}|z(t)|_{\mathbb{L}^{4}}^{4} d t<\infty$.

A precise definition of a solution to problem (4.16) will be given in section 6 , Roughly speaking a solution to problem (4.16) is a process $u(t), t \geq 0$, which can be represented in the form $u(t)=v(t)+z_{\alpha}(t)$, where $z_{\alpha}(t), t \in \mathbb{R}$, is a stationary Ornstein-Uhlenbeck process with drift $-A-\alpha I$, i.e. a stationary solution of

$$
d z+(\mathrm{A}+\alpha) z d t=d W(t), \quad t \in \mathbb{R}
$$


and $v(t), t \geq 0$, is a solution to the following problem (with $v_{0}=x-z(0)$ ):

$$
\begin{aligned}
\frac{d v}{d t} & =-\nu \mathrm{A} v-B(v)-B(v, z)-B(z, v)-B(z)+\alpha z+f, \\
v(0) & =v_{0} .
\end{aligned}
$$

In the remainder of this section we will study problem (4.19) (4.20) for fixed deterministic function $z \in L_{\mathrm{loc}}^{4}\left([0, \infty) ; \mathbb{L}^{4}(D)\right) \cap L_{\mathrm{loc}}^{2}\left([0, \infty) ; \mathrm{V}^{\prime}\right)$ and $v_{0} \in \mathrm{H}$. Motivated by Brzeźniak, Capiński and Flandoli [8] (see also Temam [4]), we have the following.

Definition 4.4. Suppose that $z \in L_{\mathrm{loc}}^{4}\left([0, \infty) ; \mathbb{L}^{4}(D)\right) \cap L_{\mathrm{loc}}^{4}([0, \infty) ; \mathrm{V}), f \in \mathrm{V}^{\prime}$ and $v_{0} \in \mathrm{H}$. A function $v \in C([0, \infty) ; \mathrm{H}) \cap L_{\mathrm{loc}}^{2}\left([0, \infty) ; \mathrm{V}^{\prime}\right) \cap L_{\mathrm{loc}}^{4}\left([0, \infty) ; \mathbb{L}^{4}(D)\right)$ is a solution to problem (4.19) -(4.20) iff $v(0)=v_{0}$ and (4.19) holds in the weak sense, i.e. for any $\phi \in \mathrm{V}$

$$
\begin{aligned}
\frac{d}{d t}(v(t), \phi)=-\nu(v(t), \mathrm{A} \phi) & -b(v(t)+z(t), \phi, v(t)+z(t)) \\
& +(\alpha z(t)+f, \phi) .
\end{aligned}
$$

Theorem 4.5. Assume that $\alpha \geq 0, z \in L_{\mathrm{loc}}^{4}\left([0, \infty) ; \mathbb{L}^{4}(D)\right) \cap L_{\mathrm{loc}}^{2}\left([0, \infty) ; \mathrm{V}^{\prime}\right)$, $v_{0} \in \mathrm{H}$ and $f \in \mathrm{V}^{\prime}$.

(i) Then there exists a unique solution $v$ of problem (4.19)-(4.20).

(ii) If in addition, $v_{0} \in \mathrm{V}, f \in \mathrm{H}$ and $z \in C(\mathbb{R} ; V) \cap L_{\text {loc }}^{2}(\mathbb{R} ; D(\mathrm{~A}))$, then $v \in C([0, \infty) ; \mathrm{V}) \cap L_{\mathrm{loc}}^{2}([0, \infty) ; D(\mathrm{~A}))$.

Part (i) of Theorem 4.5 will be proved in section [5, while for the proof of part (ii) we refer to Brzeźniak and Li [9]. The following result strengthens Lemma 5.2 in 9 .

Theorem 4.6. Assume that, for some $T>0$ fixed, $x_{n} \rightarrow x$ in $\mathrm{H}$,

$$
z_{n} \rightarrow z \text { in } L^{4}\left([0, T] ; \mathbb{L}^{4}(D)\right) \cap L^{2}\left(0, T ; \mathrm{V}^{\prime}\right), \quad f_{n} \rightarrow f \text { in } L^{2}\left(0, T ; \mathrm{V}^{\prime}\right) .
$$

Let us denote by $v(t, z) x$ the solution of problem (4.19) -(4.20) and by $v\left(t, z_{n}\right) x_{n}$ is solution of problem (4.19) -(4.20) with $z, f, x$ being replaced by $z_{n}, f_{n}, x_{n}$. Then

$$
v\left(\cdot, z_{n}\right) x_{n} \rightarrow v(\cdot, z) x \text { in } C([0, T] ; \mathrm{H}) \cap L^{2}(0, T ; \mathrm{V}) .
$$

In particular, $v\left(T, z_{n}\right) x_{n} \rightarrow v(T, z) x$ in $\mathrm{H}$.

Remark 4.7. Because the solution $v$ to problem (4.19)-(4.20) is not constructed by means of the Banach Fixed Point Theorem, the continuity result in Theorem 4.6 does not follow from Theorem 4.5 by employing the principle of smooth dependence of fixed points on parameters. See however our papers [4] and [9] for using this idea in different topologies.

\section{Proof of Theorems 4.5 and 4.6}

5.1. Proof of Theorem 4.5. There are many possible and different proofs. For example, one can follow the method from [8] and prove first the local existence with more regular initial and external data. Then, one should prove the existence of a global solution for the same regularized initial and external data. Finally, one should establish certain a priori inequalities in appropriate weaker norms, and using these, demonstrate the existence a limit of these more regular solutions when the regularization is removed. Another approach, following [23] and [21, is to prove the 
existence and the uniqueness of a local maximal solution in a space $L^{4}\left(0, T ; \mathbb{L}^{4}(\mathcal{O})\right)$ and then show that this solution is in fact more global and more regular. Neither of these approaches rely on the compactness argument but only on the Banach Fixed Point Theorem, and they work equally well in both bounded and unbounded domains. We have however chosen another, in fact more complicated, approach, because it yields estimates that are essential in proving the weak continuity of the RDS, an essential tool in our investigation; see section 7.

Let us fix $T>0$. It is enough to restrict our problem to the interval $[0, T]$. As already mentioned, we will only prove the first part of the theorem.

Part I. Existence of solutions. First let us observe that $F \in L^{2}\left(0, T ; \mathrm{V}^{\prime}\right)$ from Lemma 4.2 and because $z \in L_{\text {loc }}^{4}\left([0, \infty) ; \mathbb{L}^{4}(D)\right) \cap L_{\text {loc }}^{2}\left([0, \infty) ; \mathrm{V}^{\prime}\right)$, where $F(t)=$ $B(z(t))+\alpha z(t)+f, t \geq 0$. Second, since $\mathrm{V}$ is a separable Hilbert space, $\mathcal{V}$ is dense in $\mathrm{V}$ and $\mathrm{V}$ is dense in $\mathrm{H}$, we can find a sequence $\left\{w_{j}\right\}_{j \in \mathbb{N}}$ in $\mathcal{V}$ which is free and total in $\mathrm{V}$ and which is an orthonormal basis of $\mathrm{H}$.

Let us denote $\mathrm{H}_{m}=\operatorname{span}\left\{w_{1}, w_{2}, \cdots, w_{m}\right\}$ with the norm inherited from $\mathrm{H}$ and $\mathrm{V}_{m}=\operatorname{span}\left\{w_{1}, w_{2}, \cdots, w_{m}\right\}$ with the norm inherited from V. Denote also by $P_{m}$ the orthogonal projection from $\mathrm{H}$ onto $\mathrm{H}_{m}$. Consider the following approximate equation for problem (4.19)-4.20 on the finite-dimensional space $\mathrm{H}_{m}$ :

$$
\left\{\begin{aligned}
\frac{d v_{m}}{d t} & \left.=P_{m}\left[-\nu \mathrm{A} v_{m}-B\left(v_{m}\right)-B\left(v_{m}, z\right)-B\left(z, v_{m}\right)\right)+F\right], \\
v_{m}(0) & =P_{m} v(0) .
\end{aligned}\right.
$$

Let $j_{m}: \mathrm{H}_{m} \hookrightarrow \mathrm{H}$ be the natural embedding, $\mathrm{A}_{m}:=P_{m} \mathrm{~A} J_{m}, B_{m}=P_{m} B\left(j_{m}, j_{m}\right)$ and for $t \in[0, T], D_{m}(t)=P_{m} B\left(j_{m}, z(t)\right), E_{m}(t)=P_{m} B\left(z(t), j_{m}\right), F_{m}(t)=$ $P_{m} F(t)$.

One easily shows that $\mathrm{A}_{m}$, resp. $B_{m}$, is a continuous linear, resp. bilinear, map in $H_{m}$. Moreover, for $t \in[0, T], D_{m}(t)$ and $E_{m}(t)$ are bounded linear maps in $\mathrm{H}_{m}$, and with some universal (hence independent of $m$ ) positive constant $C$,

$$
\left|D_{m}(t)\right|_{\mathcal{L}\left(\mathrm{H}_{m}, \mathrm{H}_{m}\right)} \leq C|z(t)|_{\mathbb{L}^{4}}, \quad\left|E_{m}(t)\right|_{\mathcal{L}\left(\mathrm{H}_{m}, \mathrm{H}_{m}\right)} \leq C|z(t)|_{\mathbb{L}^{4}}, \quad t \in[0, T] .
$$

Denoting $G_{m}(t, x)=-\nu \mathrm{A}_{m} x-B_{m}(x)+D_{m}(t)(x)+E_{m}(t)(x)+F_{m}(t), x \in \mathrm{H}_{m}$, $t \in[0, T]$ and $x_{0}=P_{m} v(0)$, we see that problem (5.1) takes the following form:

$$
\left\{\begin{aligned}
\frac{d x}{d t} & =G(t, x(t)), \quad t \geq 0 \\
x(0) & =x_{0} .
\end{aligned}\right.
$$

The preceding observations imply that the maps $G(t, \cdot)$ are locally Lipschitz in the following sense. For each $R>0$, there exists a positive function $C=$ $C_{R} \in L^{1}(0, T)$, such that for any $t \in[0, T]$ and $|x|,|y| \leq R,|G(t, x)-G(t, y)| \leq$ $C_{R}(t)|x-y|$. Therefore, by the local existence and uniqueness theorem for ordinary differential equations on Banach spaces (see Cartan [13]), there exists a number $T_{m} \in(0, T]$ and a function $x:\left[0, T_{m}\right) \rightarrow \mathrm{H}_{m}$ which is a unique local maximal solution of the above problem. In particular, $\lim \sup _{t} T_{m}\left|v_{m}(t)\right|=\infty$ if $T_{m}<T$. Hence, in order to prove that $T_{m}=T$ it is sufficient to establish that $\lim \sup _{t \nearrow T_{m}}\left|v_{m}(t)\right|<\infty$. This is a direct consequence of the a priori estimates we are going to establish in what follows. For this we need to recall Lemma III.1.2 from 40. 
Lemma 5.1. Suppose that $\mathrm{V} \subset \mathrm{H} \cong \mathrm{V}^{\prime} \subset \mathrm{V}^{\prime}$ is a Gelfand triple of Hilbert spaces. If a function $u$ belongs to $L^{2}(0, T ; \mathrm{V})$ and its weak derivative belongs to $L^{2}\left(0, T ; \mathrm{V}^{\prime}\right)$, then $u$ is a.e. equal to a continuous function from $[0, T]$ to $\mathrm{H}$, the real-valued function $|u|^{2}$ is absolutely continuous and, in the weak sense on $(0, T)$, one has (with $\langle\cdot, \cdot\rangle$ being the duality between $\mathrm{V}^{\prime}$ and $\mathrm{V}$ )

$$
\frac{d}{d t}|u(t)|^{2}=2\left\langle\frac{d}{d t} u(t), u(t)\right\rangle .
$$

Let us observe that by (4.9)

$$
\left(-\nu \mathrm{A}_{m} v_{m}, v_{m}\right)=-\nu\left(P_{m} \mathrm{~A} v_{m}, v_{m}\right)=-\nu\left(\mathrm{A} v_{m}, v_{m}\right)=-\nu\left\|v_{m}\right\|^{2}
$$

and by (4.10)

$$
\left(B_{m} v_{m}, v_{m}\right)=\left(P_{m} B\left(v_{m}\right), v_{m}\right)=\left(B\left(v_{m}\right), v_{m}\right)=0, \quad\left(E_{m}(t) v_{m}, v_{m}\right)=0 .
$$

Therefore, applying the above Lemma 5.1 with the triple $\mathrm{V}_{m}, \mathrm{H}_{m}, \mathrm{~V}_{m}^{\prime}$ and invoking equation (5.2), we have on $\left[0, T_{m}\right)$

$$
\frac{1}{2} \frac{d}{d t}\left|v_{m}(t)\right|^{2}=-\nu\left\|v_{m}(t)\right\|-b\left(v_{m}(t), v_{m}(t), z(t)\right)+\left\langle F(t), v_{m}(t)\right\rangle .
$$

Then combining (4.11) with (4.14), and next using (4.12), (4.13) and the Young inequality, we infer that

$$
\frac{d}{d t}\left|v_{m}(t)\right|^{2}+\nu\left\|v_{m}(t)\right\|^{2} \leq \frac{C}{\nu}|z(t)|_{\mathbb{L}^{4}(D)}^{4}\left|v_{m}(t)\right|^{2}+\frac{2}{\nu}|F(t)|_{\mathrm{V}^{\prime}}^{2}, \quad t \in\left[0, T_{m}\right) .
$$

Next applying the Gronwall Lemma and denoting $\Psi_{T}(z)=e^{\int_{0}^{T} \frac{C}{\nu}|z(\tau)|_{\mathbb{L}^{4}(D)}^{4} d \tau}<\infty$, $C_{F}=\frac{2}{\nu} \int_{0}^{T}|F(s)|_{\mathrm{V}^{\prime}}^{2} d s<\infty$, we find that

$$
\begin{aligned}
\left|v_{m}(t)\right|^{2} & \leq\left|v_{m}(0)\right|^{2} e^{\int_{0}^{t} \frac{C}{\nu}|z(\tau)|_{\mathbb{L}^{4}(D)}^{4} d \tau}+\int_{0}^{t} \frac{2}{\nu}|F(s)|_{\mathrm{V}^{\prime}}^{2} e^{-\int_{t}^{s} \frac{C}{\nu}|z(\tau)|_{\mathbb{L}^{4}(D)}^{4} d \tau} d s \\
& \leq \Psi_{T}(z)\left|v_{m}(0)\right|^{2}+C_{F} \leq \Psi_{T}(z)|v(0)|^{2}+C_{F}<\infty, \quad t \in\left[0, T_{m}\right) .
\end{aligned}
$$

Therefore,

$$
\sup _{t \in\left[0, T_{m}\right)}\left|v_{m}(t)\right|^{2} \leq \Psi_{T}(z)|v(0)|^{2}+C_{F},
$$

which, on the one hand implies that $T_{m}=T$, and on the other hand implies that

$$
\text { the sequence }\left\{v_{m}\right\} \text { is bounded in } L^{\infty}(0, T ; \mathrm{H}) \text {. }
$$

In order to get another a priori estimate we integrate equation (5.4) from 0 to $T$ and by using (5.5) we obtain

$$
\begin{aligned}
& \left|v_{m}(T)\right|^{2}+\nu \int_{0}^{T}\left\|v_{m}(t)\right\|^{2} d t \\
& \leq \frac{C}{\nu} \int_{0}^{T}|z(t)|_{\mathbb{L}^{4}(D)}^{4}\left|v_{m}(t)\right|^{2} d t+\frac{2}{\nu} \int_{0}^{T}|F(t)|_{\mathrm{V}^{\prime}}^{2} d t+\left|v_{m}(0)\right|^{2} \\
& \leq \frac{C}{\nu}\left(\Psi_{T}(z)|v(0)|^{2}+C_{F}\right) \int_{0}^{T}|z(t)|_{\mathbb{L}^{4}(D)}^{4} d t+\frac{2}{\nu} \int_{0}^{T}|F(t)|_{\mathrm{V}^{\prime}}^{2} d t+|v(0)|^{2} .
\end{aligned}
$$


The last inequality implies that

$$
\text { the sequence }\left\{v_{m}\right\} \text { is bounded in } L^{2}(0, T ; \mathrm{V}) \text {. }
$$

Let us point out that claims (5.6) and (5.7) are sufficient to infer that the sequence $\left\{v_{m}\right\}$ has a convergent subsequence. However, in order to show that the limit function $v$ is a solution to our problem, we need to show that $v_{m}$ converges to $v$ in the strong and not simply the weak topology. This can be done, as in the case of the classical NSEs, in many different ways. Here we feel that the fractional derivative method (see [40, ch. III.2) is best suited for the unbounded domain case. We begin with an observation that (5.1) together with (4.13) imply that

$$
\text { the sequence }\left\{v_{m}^{\prime}\right\} \text { is bounded in } L^{2}\left(0, T ; \mathrm{V}^{\prime}\right) \text {. }
$$

For example we shall prove now that $\sup _{m \in \mathbb{N}} \int_{0}^{T}\left|D_{m}(t) v_{m}(t)_{m}(t)\right|_{\mathrm{V}^{\prime}}^{2} d t<\infty$. From (4.13), (4.12) and assumptions on $z$, we have

$$
\begin{aligned}
& \int_{0}^{T}\left|D_{m}(t) v_{m}(t)\right|_{\mathrm{V}^{\prime}}^{2} d t \leq \int_{0}^{T}\left|v_{m}(t)\right|_{\mathbb{L}^{4}}|z(t)|_{\mathbb{L}^{4}} d t \\
& \leq\left|v_{m}\right|_{L^{\infty}(0, T ; \mathrm{H})}\left(\int_{0}^{T}\left|\nabla v_{m}(t)\right|^{2} d t\right)^{1 / 2}\left(\int_{0}^{T}|z(t)|_{\mathbb{L}^{4}}^{4} d t\right)^{1 / 2} .
\end{aligned}
$$

Hence, in view of (5.6) and (5.7), $\sup _{m \in \mathbb{N}} \int_{0}^{T}\left|D_{m}(t) v_{m}(t)\right|_{\mathrm{V}^{\prime}}^{2} d t<\infty$.

Let us denote by $\mathcal{H}^{\gamma, 2}(\mathbb{R} ; \mathrm{V}, \mathrm{H})$ (see e.g. [40] or [34) a Hilbert space consisting of all $v \in L^{2}(\mathbb{R} ; \mathrm{V})$ such that, with $\hat{v}$ being the Fourier transform of $v$,

$$
\int_{\mathbb{R}}\left|(i \tau)^{\gamma} \hat{v}(\tau)\right|_{\mathrm{H}}^{2} d \tau<\infty
$$

If we put $\tilde{v}_{m}=1_{(0, T)} v_{m}$, then from (5.7) and (5.8) we infer (see 34]) that for $\gamma \leq 1 / 2$,

$$
\text { the sequence }\left\{\tilde{v}_{m}(\cdot)\right\} \text { is bounded in } \mathcal{H}^{\gamma, 2}(\mathbb{R} ; \mathrm{V}, \mathrm{H}) \text {. }
$$

Because of (5.6) and (5.7), without loss of generality we may assume that there exists $v \in L^{2}(0, T ; \mathrm{V}) \cap L^{\infty}(0, T ; \mathrm{H})$ such that

$$
\begin{cases}v_{m} \rightarrow v, & \text { weakly in } L^{2}(0, T ; \mathrm{V}), \\ v_{m} \rightarrow v, & \text { weakly-star in } L^{\infty}(0, T ; \mathrm{H}) .\end{cases}
$$

Since for any $R>0$, the set $D_{R}=B(0, R) \cap D$ is bounded, the embedding $\mathbb{H}^{1,2}\left(D_{R}\right) \hookrightarrow \mathbb{L}^{2}\left(D_{R}\right)$ is compact, and since by (5.10)

$$
\left.\tilde{v}_{m}(\cdot)\right|_{D_{R}} \text { is bounded in } \mathcal{H}^{\gamma, 2}\left(0, T ; \mathbb{H}^{1,2}\left(D_{R}\right), \mathbb{L}^{2}\left(D_{R}\right)\right) \text {. }
$$

Since, by the compactness theorem from [40] (see Theorem III.2.2) the imbedding $\mathcal{H}^{\gamma, 2}\left(0, T ; \mathbb{H}^{1}\left(D_{R}\right), \mathbb{L}^{2}\left(D_{R}\right)\right) \hookrightarrow L^{2}\left(0, T ; \mathbb{L}^{2}\left(D_{R}\right)\right)$ is compact, we may deduce from (5.12) that for each $R>0$ we can find a subsequence of $\left\{v_{m}\right\}$ (which for the sake of simplicity of notation will be denoted as the whole sequence), such that

$$
\left.\left.v_{m}\right|_{D_{R}} \rightarrow v\right|_{D_{R}}, \quad \text { strongly in } L^{2}\left(0, T ; \mathbb{L}^{2}\left(D_{R}\right)\right) .
$$


By considering $R=1,2, \cdots$ and applying the standard diagonal procedure, we can find a subsequence $\left\{v_{m}\right\}$ (which again will be denoted as the whole sequence), such that

$$
v_{m} \rightarrow v \quad \text { strongly in } \quad L^{2}\left(0, T ; \mathbb{L}_{\text {loc }}^{2}(D)\right) .
$$

It remains to show that $v \in C([0, T] ; \mathrm{H})$ and that $v$ is a solution to problem (4.19)(4.20). To prove the latter we take a continuously differentiable function $\psi:[0, T] \rightarrow$ $\mathbb{R}$ such that $\psi(T)=0$. Let $\phi \in \mathrm{H}_{n}$ for some $n \in \mathbb{N}$. Then, by taking the H-scalar product of (5.2) with function $\psi(\cdot) \phi$ and then integrating by parts, we get

$$
\begin{aligned}
& -\int_{0}^{T}\left(v_{m}(t), \psi^{\prime}(t) \phi\right) d t=-\nu \int_{0}^{T}\left(\mathrm{~A}_{m} v_{m}(t), \psi(t) \phi\right) d t \\
& \quad+\int_{0}^{T}\left(B_{m}\left(v_{m}(t)\right), \psi(t) \phi\right) d t+\int_{0}^{T}\left(D_{m}(t) v_{m}(t), \psi(t) \phi\right) d t \\
& \quad+\int_{0}^{T}\left(E_{m}(t) v_{m}(t), \psi(t) \phi\right) d t+\int_{0}^{T}\left\langle F_{m}(t), \psi(t) \phi\right\rangle d t+\left(v_{m}(0), \psi(0) \phi\right) .
\end{aligned}
$$

Our aim now is to pass to the limit in (5.14) when $m \rightarrow \infty$. From the choice of $\phi$ we can find $R \in \mathbb{N}$ such that $\operatorname{supp} \phi \subset B(0, R) \cap D=D_{R}$. By (5.13), we have

$$
\left.\left.v_{m}\right|_{D_{R}} \rightarrow v\right|_{D_{R}} \quad \text { strongly in } L^{2}\left(0, T ; \mathbb{L}^{2}\left(D_{R}\right)\right) .
$$

Since $\psi(\cdot) \phi \in L^{2}\left(0, T ; \mathbb{L}^{2}\left(D_{R}\right)\right)$ and

$$
\left(v_{m}(t)-v(t), \psi^{\prime}(t)(t) \phi\right)=\left(v_{m}(t)-v(t), \psi^{\prime}(t) \phi\right)_{\mathbb{L}^{2}\left(D_{R}\right)},
$$

in view of (5.15) by means of the Cauchy-Schwartz inequality, we infer that

$$
\int_{0}^{T}\left(v_{m}(t)-v(t), \psi^{\prime}(t) \phi\right) d t \rightarrow 0
$$

Therefore, the left-hand side of (5.14) converges to $-\int_{0}^{T}\left(v(t), \psi^{\prime}(t) \phi\right) d t$.

Next let us take $m \geq n$ so that $\mathrm{H}_{n} \subset \mathrm{H}_{m}$ and $P_{m} \phi=\phi$. In order to deal with the first term on the RHS of (5.14), we observe that

$$
\begin{aligned}
& \int_{0}^{T}\left(\mathrm{~A}_{m} v_{m}(t), \psi(t) \phi\right) d t=\int_{0}^{T}\left(P_{m} \mathrm{~A} v_{m}(t), \psi(t) \phi\right) d t \\
& =\int_{0}^{T}\left(\mathrm{~A} v_{m}(t), \psi(t) P_{m} \phi\right) d t=\int_{0}^{T}\left(\mathrm{~A} v_{m}(t), \psi(t) \phi\right) d t=\int_{0}^{T}\left(\left(v_{m}(t), \psi(t) \phi\right)\right) d t
\end{aligned}
$$

Since $\psi(\cdot) \phi \in L^{2}(0, T ; \mathrm{V})$, it follows from (5.11) that, as $m \rightarrow \infty$,

$$
\int_{0}^{T}\left(\mathrm{~A}_{m} v_{m}(t), \psi(t) \phi\right) d t-\int_{0}^{T}((v(t), \psi(t) \phi)) d t=\int_{0}^{T}\left(\left(v_{m}(t)-v(t), \psi(t) \phi\right)\right) d t \rightarrow 0 .
$$

With regard to the third term on the RHS (5.14), since

$$
\begin{aligned}
& \int_{0}^{T}\left(D_{m}(t) v_{m}(t), \psi(t) \phi\right) d t=\int_{0}^{T}\left(P_{m} B\left(v_{m}(t), z(t)\right), \psi(t) \phi\right) d t \\
= & \int_{0}^{T} b\left(v_{m}(t), z(t), \psi(t) P_{m} \phi\right) d t=\int_{0}^{T} b\left(v_{m}(t), z(t), \psi(t) \phi\right) d t,
\end{aligned}
$$


by estimates similar to those in (5.9), we have

$$
\begin{aligned}
& \left|\int_{0}^{T}\left(D_{m}(t) v_{m}(t), \psi(t) \phi\right) d t-\int_{0}^{T} b(v(t), z(t), \psi(t) \phi) d t\right| \\
& =\left|\int_{0}^{T} b\left(v_{m}(t)-v(t), z(t), \psi(t) \phi\right) d t\right| \int_{0}^{T}\left|v_{m}(t)-v(t)\right|_{\mathbb{L}^{4}}|z(t)|_{\mathbb{L}^{4}}|\nabla \psi(t) \phi|_{\mathbb{L}^{2}} d t \\
& \leq C\left|v_{m}-v\right|_{L^{\infty}(0, T ; \mathrm{H})}^{1 / 2}|| v_{m}(t)-\left.v(t)\right|_{L^{2}(0, T ; \mathrm{V})} ^{1 / 2} \rightarrow 0 .
\end{aligned}
$$

Analogously, we have $\int_{0}^{T}\left(E_{m}(t) v_{m}(t), \psi(t) \phi\right) d t-\int_{0}^{T} b(z(t), v(t), \psi(t) \phi) d t \rightarrow 0$.

As to the fifth term, we have $\int_{0}^{T}\left\langle F_{m}(t), \psi(t) \phi\right\rangle d t=\int_{0}^{T}\langle F(t), \psi(t) \phi\rangle d t$.

In order to be able to deal with the second, i.e. the nonlinear, term on the RHS of (5.14), we need the following version of Lemma 3.2 from ch. III of [40], the proof of which is omitted.

Lemma 5.2. Suppose that $D_{1}$ is a bounded subset of $D$, and $u:[0, T] \times D \rightarrow \mathbb{R}^{2}$ is a $C^{1}$-class function such that $\operatorname{supp} u(t, \cdot) \subset D_{1}$ for $t \in[0, T]$, and

$$
\sup _{i, j} \sup _{(t, x) \in[0, T] \times D_{1}}\left|D_{i} u^{j}(t, x)\right|=C<\infty .
$$

Suppose also that $v_{m} \rightarrow v$ weakly in $L^{2}(0, T ; \mathrm{V})$ and strongly in $L^{2}\left(0, T ; \mathbb{L}^{2}\left(D_{1}\right)\right)$. Then,

$$
\int_{0}^{T} b\left(v_{m}(t), v_{m}(t), u(t)\right) d t \rightarrow \int_{0}^{T} b(v(t), v(t), u(t)) d t
$$

In the following sections we will need the following extension of the previous lemma.

Corollary 5.3. If $\left\{v_{m}\right\}_{m}$ is bounded in $L^{\infty}(0, T ; \mathrm{H}), v \in L^{\infty}(0, T ; \mathrm{H})$, and $v_{m} \rightarrow$ $v$ weakly in $L^{2}(0, T ; \mathrm{V})$ and strongly in $L^{2}\left(0, T ; \mathbb{L}_{\mathrm{loc}}^{2}(D)\right)$. Then for any $w \in$ $L^{4}\left(0, T ; \mathbb{L}^{4}(D)\right)$,

$$
\int_{0}^{T} b\left(v_{m}(t), v_{m}(t), w(t)\right) d t \rightarrow \int_{0}^{T} b(v(t), v(t), w(t)) d t
$$

Proof. From our assumptions, there exists a constant $C>0$, such that

$$
\sup _{0 \leq t \leq T}\left|v_{m}(t)\right|+\sup _{0 \leq t \leq T}|v(t)|^{\frac{1}{2}}\left(\int_{0}^{T}\left\|v_{m}(t)\right\|^{2} d t\right)^{\frac{3}{4}}+\left(\int_{0}^{T}\|v(t)\|^{2} d t\right)^{\frac{3}{4}} \leq C .
$$

Let us choose $\varepsilon>0$. Since $w \in L^{4}\left(0, T ; \mathbb{L}^{4}(D)\right)$, by standard regularization methods we can find a function $u$ satisfying the assumptions of the previous lemma and such that $\left(\int_{0}^{T}|w(s)-u(s)|_{\mathbb{L}^{4}}^{4} d s\right)^{\frac{1}{4}}<\frac{\varepsilon}{3 C^{2}}$. Hence, by this lemma, we can find $M_{\varepsilon} \in \mathbb{N}$ such that for $m \geq M_{\varepsilon},\left|\int_{0}^{T} b\left(v_{m}(t), v_{m}(t), u(t)\right) d t-\int_{0}^{T} b(v(t), v(t), u(t)) d t\right|<\frac{\varepsilon}{3}$. 
Hence, because of inequalities (4.13) and (4.12) we have, for $m>M_{\varepsilon}$,

$$
\begin{aligned}
& \begin{array}{l}
\left|\int_{0}^{T} b\left(v_{m}(t), v_{m}(t), w(t)\right) d t-\int_{0}^{T} b(v(t), v(t), w(t)) d t\right| \\
=\mid \int_{0}^{T} b\left(v_{m}(t), v_{m}(t), w(t)-u(t)\right) d t+\int_{0}^{T} b\left(v_{m}(t), v_{m}(t), u(t)\right) d t \\
\quad-\int_{0}^{T} b(v(t), v(t), w(t)-u(t)) d t-\int_{0}^{T} b(v(t), v(t), u(t)) d t \mid \\
\leq\left|\int_{0}^{T} b\left(v_{m}(t), v_{m}(t), w(t)-u(t)\right) d t\right|+\left|\int_{0}^{T} b(v(t), v(t), w(t)-u(t)) d t\right| \\
\quad+\left|\int_{0}^{T} b\left(v_{m}(t), v_{m}(t), u(t)\right) d t-\int_{0}^{T} b(v(t), v(t), u(t)) d t\right| \\
\leq \frac{\varepsilon}{3}+\int_{0}^{T}\left|v_{m}(t)\right|^{\frac{1}{2}}\left\|v_{m}(t)\right\|^{\frac{3}{2}}|w(t)-u(t)|_{\mathbb{L}^{4}} d t \\
\quad+\int_{0}^{T}|v(t)|^{\frac{1}{2}}\|v(t)\|^{\frac{3}{2}}|w(t)-u(t)|_{\mathbb{L}^{4}} d t \\
\leq \frac{\varepsilon}{3}+\sup _{0 \leq t \leq T}\left|v_{m}(t)\right|^{\frac{1}{2}}\left(\int_{0}^{T}\left\|v_{m}(t)\right\|^{\frac{3}{2}} d t\right)^{\frac{3}{4}}\left(\int_{0}^{T}|w(t)-u(t)|_{\mathbb{L}^{4}}^{4} d t\right)^{\frac{1}{4}} \\
\quad+\sup _{0 \leq t \leq T}|v(t)|^{\frac{1}{2}}\left(\int_{0}^{T}\|v(t)\|^{\frac{3}{2} \frac{4}{3}} d t\right)^{\frac{3}{4}}\left(\int_{0}^{T}|w(t)-u(t)|_{\mathbb{L}^{4}}^{4} d t\right)^{\frac{1}{4}}<\varepsilon,
\end{array}
\end{aligned}
$$

which concludes the proof.

By applying Lemma 5.2 to the second term on the RHS of (5.14) with $u(t, x)=$ $\psi(t) \phi(x), t \in[0, T], x \in D$, and noting that $\left(B_{m}\left(v_{m}\right), \psi \phi\right)=\left(P_{m} B\left(v_{m}, v_{m}\right), \psi \phi\right)=$ $\left(B\left(v_{m}, v_{m}\right), \psi P_{m} \phi\right)=\left(B\left(v_{m}, v_{m}\right), \psi \phi\right)=b\left(v_{m}, v_{m}, \psi \phi\right)$, we have the following convergence:

$$
\begin{gathered}
\int_{0}^{T}\left(B_{m}\left(v_{m}(t)\right), \psi(t) \phi\right) d t=\int_{0}^{T} b\left(v_{m}(t), v_{m}(t), \psi(t) \phi\right) d t \\
\rightarrow \int_{0}^{T} b(v(t), v(t), \psi(t) \phi) d t .
\end{gathered}
$$

Hence, by taking the $m \rightarrow \infty$ limit in (5.14), we arrive at

$$
\begin{aligned}
&-\int_{0}^{T}\left(v(t), \psi^{\prime}(t) \phi\right) d t=-\nu \int_{0}^{T}((v(t), \psi(t) \phi)) d t \\
& \quad+\int_{0}^{T} b(v(t), v(t), \psi(t) \phi) d t+\int_{0}^{T} b(v(t), z(t), \psi(t) \phi) d t \\
& \quad+\int_{0}^{T} b(z(t), v(t), \psi(t) \phi) d t+\int_{0}^{T}\langle F(t), \psi(t) \phi\rangle d t+\left(v_{0}, \phi\right) \psi(0) .
\end{aligned}
$$

Since (5.16) has been proved for any $\phi \in \bigcup_{n=1}^{\infty} \mathrm{H}_{n}$ and the set $\bigcup_{n=1}^{\infty} \mathrm{H}_{n}$ is a dense subspace of $\mathrm{V}$, by using a standard continuity argument we can show that (5.16) holds for any $\phi \in \mathrm{V}$ and any $\psi \in C_{0}^{1}([0, T))$. In particular, it is satisfied for all $\psi \in C_{0}^{1}(0, T)$. Hence, $v$ solves problem (4.21) and hence it satisfies equation (4.19).

Now we will show that $v \in C([0, T], \mathrm{H})$. Since $v$ solves (4.19) $), v \in L^{2}(0, T ; \mathrm{V})$ and $\mathrm{A}: \mathrm{V} \rightarrow \mathrm{V}^{\prime}$ is a bounded linear operator, $\mathrm{A} v \in L^{2}\left(0, T ; \mathrm{V}^{\prime}\right)$. Because 
$z \in L_{\text {loc }}^{4}\left([0, \infty) ; \mathbb{L}^{4}(D)\right) \cap L_{\text {loc }}^{2}\left([0, \infty) ; \mathrm{V}^{\prime}\right)$, it follows from Lemma 4.2 that not only $B(z)+\alpha z+f \in L^{2}\left(0, T ; \mathrm{V}^{\prime}\right)$ but also $B(v), B(v, z), B(z, v)$ all belong to $L^{2}\left(0, T ; \mathrm{V}^{\prime}\right)$ as well. Hence $v^{\prime} \in L^{2}\left(0, T ; \mathrm{V}^{\prime}\right)$. Thus, it follows from a trace theorem (see e.g. Theorem 1.3.1 in [34] or Lemma III.1.2 in [40]) that $v \in C([0, T] ; \mathrm{H})$.

Next, we will show that our solution $v$ satisfies (4.20), i.e. $v(0)=v_{0}$. Recall that $v \in L^{2}(0, T ; \mathrm{V}) \cap C([0, T] ; \mathrm{H}), v^{\prime} \in L^{2}\left(0, T ; \mathrm{V}^{\prime}\right)$ and $v$ satisfies (4.19). Let us choose arbitrary $\phi \in \mathrm{V}$ and $\psi \in C_{0}^{1}([0, T))$ such that $\psi(0)=1$. By multiplying equation (4.19) by $\psi(t) \phi$ and then by integrating by parts, we obtain

$$
\begin{aligned}
-\int_{0}^{T} & (v(t), \phi) \psi^{\prime}(t) d t=-\nu \int_{0}^{T}((v(t), \phi)) \psi(t) d t \\
& +\int_{0}^{T} b(v(t), v(t), \phi) \psi(t) d t+\int_{0}^{T} b(v(t), z(t), \phi) \psi(t) d t \\
& +\int_{0}^{T} b(z(t), v(t), \phi) \psi(t) d t+\int_{0}^{T}\langle\tilde{f}(t), \phi\rangle \psi(t) d t+(v(0), \phi) \psi(0) .
\end{aligned}
$$

By comparing equality (5.16) to (5.17) we infer that $\left(v_{0}-v(0), \phi\right) \psi(0)=0$. Since $\psi(0)=1$ we infer that $\left(v_{0}-v(0), \phi\right)=0$, for any $\phi \in \mathrm{V}$. Hence, the density of $\mathrm{V}$ in $\mathrm{H}$ implies that $v(0)=v_{0}$.

Since we proved that the solution $v$ belongs to $L^{2}(0, T ; \mathrm{V}) \cap C([0, T] ; \mathrm{H})$ we infer by employing inequality (4.12) that $v$ belongs as well to $L^{4}\left(0, T ; \mathbb{L}^{4}(D)\right)$.

Part II. Uniqueness of solutions. We argue as in the proof of the uniqueness due to Lions-Prodi [35]; see also Theorem III.3.2 in [40. Let us assume that $v_{1}$ and $v_{2}$ are two solutions of (4.19)-(4.20), and we put $w=v_{1}-v_{2}$. Then, by definition both $v_{1}$ and $v_{2}$ (and hence $w$ as well) belong to $L^{2}(0, T ; \mathrm{V}) \cap C([0, T], \mathrm{H})$, and by the argument above their (weak-) time derivatives belong to $L^{2}\left(0, T ; \mathrm{V}^{\prime}\right)$. Moreover, $w$ solves the following:

$$
\left\{\begin{aligned}
\frac{d w}{d t}+\nu \mathrm{A} w & =B(w, z)-B(z, w)-B\left(w, v_{1}\right)-B\left(v_{1}, w\right), \\
w(0) & =0 .
\end{aligned}\right.
$$

The regularity of $w$ allows us to apply Lemma III.1.2 in [40], and hence we get

$$
\frac{d}{d t}|w(t)|^{2}+2 \nu\|w(t)\|^{2}=2 b(w(t), z(t), w(t))+2 b(w(t), u(t), w(t)) .
$$

By using inequalities (4.12) and (4.12), and then the standard Young inequality, we get

$$
\begin{aligned}
\frac{d}{d t}|w(t)|^{2}+2 \nu\|w(t)\|^{2} & \leq 2^{5 / 4}|w(t)|^{1 / 2}\|w(t)\|^{3 / 2}\left(\left|v_{1}(t)\right|_{\mathbb{L}^{4}}+|z(t)|_{\mathbb{L}^{4}}\right) \\
& \leq \frac{3 \nu}{4}\|w(t)\|^{2}+\frac{2^{6}}{\nu^{3}}|w(t)|^{2}\left(\left|v_{1}(t)\right|_{\mathbb{L}^{4}}^{4}+|z(t)|_{\mathbb{L}^{4}}^{4}\right) .
\end{aligned}
$$

Therefore,

$$
\frac{d}{d t}|w(t)|^{2} \leq \frac{64}{\nu^{3}}|w(t)|^{2}\left(\left|v_{1}(t)\right|_{\mathbb{L}^{4}}^{4}+|z(t)|_{\mathbb{L}^{4}}^{4}\right) \text { a.e. on }(0, T) .
$$

Since $\int_{0}^{T}\left(\left|v_{1}(t)\right|_{\mathbb{L}^{4}}+|z(t)|_{\mathbb{L}^{4}}\right)^{4} d t<\infty$ and $w(0)=0$, by applying the Gronwall Lemma, we infer that $|w(t)|^{2}=0$ for all $t \in[0, T]$. This means that $u(t)=v(t)$ for all $t \in[0, T]$, which proves the uniqueness. 
As a byproduct of the above proof we have the following result.

Proposition 5.4. Assume that $a<b \in \mathbb{R},\left\{x_{k}\right\}$ is a bounded sequence in $\mathrm{H}$ and $\left\{f_{k}\right\}$ is a bounded sequence in $L^{2}\left(a, b ; \mathrm{V}^{\prime}\right)$. Let $v_{k}$ be a solution of equation (4.21) on $[a, b]$ with $f$ being replaced by $f_{k}$ and with the initial condition $v_{k}(a)=x_{k}$. If $0<\gamma \leq \frac{1}{2}$, then the sequence $\left\{v_{k}\right\}$ is bounded in $L^{2}(a, b ; \mathrm{V}) \cap L^{\infty}(a, b ; \mathrm{H}) \cap$ $\mathcal{H}^{\gamma, 2}(a, b ; \mathrm{V}, \mathrm{H})$ and the sequence $\left\{v_{k}^{\prime}\right\}$ is bounded in $L^{2}\left(a, b ; \mathrm{V}^{\prime}\right)$.

5.2. Proof of Theorem 4.6. In order to simplify the proof, we introduce the following notation:

$$
\begin{aligned}
& v_{n}(t)=v\left(t, z_{n}\right), \quad v(t)=v(t, z), \quad y_{n}(t)=v\left(t, z_{n}\right)-v(t, z), \quad t \in[0, T], \\
& \hat{z}_{n}=z_{n}-z, \quad \hat{f}_{n}=f_{n}-f .
\end{aligned}
$$

It is easy to see that $y_{n}$ solves the following initial value problem:

$$
\left\{\begin{array}{l}
\frac{d y_{n}(t)}{d t}=-\nu A y_{n}(t)-B\left(v_{n}(t)+z_{n}(t)\right)-B(v(t)+z(t))+\alpha \hat{z}(t)+\hat{f}(t) \\
y_{n}(0)=x_{n}-x
\end{array}\right.
$$

Since $y_{n} \in L^{2}\left(0, T ; \mathrm{V}\right.$ and $y_{n} \in L^{2}\left(0, T ; \mathrm{V}^{\prime}\right)$, it follows from Lemma 5.1 that the function $\left|y_{n}\right|^{2}$ is absolutely continuous and in the weak sense on $(0, T), \frac{1}{2} \frac{d}{d t}\left|y_{n}(t)\right|^{2}=$ $\left\langle\frac{d}{d t} y_{n}(t), y_{n}(t)\right\rangle$. Moreover, by (4.9) we have $\left\langle A y_{n}(t), y_{n}(t)\right\rangle=\left|\nabla y_{n}(t)\right|^{2}$ a.e. on $(0, T)$. Therefore, because of (4.11) from the first equation in (5.18) we get

$$
\begin{aligned}
\frac{1}{2} \frac{d}{d t}\left|y_{n}(t)\right|^{2} & +\nu\left|\nabla y_{n}(t)\right|^{2}=b\left(y_{n}(t), v_{n}(t), y_{n}(t)\right)+b\left(y_{n}(t), z_{n}(t), y_{n}(t)\right) \\
& +b\left(v(t), \hat{z}_{n}(t), y_{n}(t)\right)+b\left(\hat{z}_{n}(t), v_{n}(t), y_{n}(t)\right) \\
& +b\left(\hat{z}_{n}(t), z_{n}(t), y_{n}(t)\right)+b\left(z(t), \hat{z}_{n}(t), y_{n}(t)\right) \\
& +\alpha\left(\hat{z}_{n}(t), y_{n}(t)\right)+\left(\hat{f}_{n}(t), y_{n}(t)\right), \quad t \geq 0
\end{aligned}
$$

By using the Young inequality, in view of inequalities (4.12) and (4.13), we infer that

$$
\begin{aligned}
& b\left(y_{n}(t), v_{n}(t), y_{n}(t)\right) \leq\left|y_{n}(t)\right|_{L^{4}}^{2}\left|\nabla v_{n}(t)\right| \leq\left|y_{n}(t)\right|\left|\nabla y_{n}(t)\right|\left|\nabla v_{n}(t)\right| \\
& \leq \frac{\nu}{16}\left|\nabla y_{n}(t)\right|^{2}+\frac{4}{\nu}\left|\nabla v_{n}(t)\right|^{2}\left|y_{n}(t)\right|^{2}, \\
& b\left(y_{n}(t), z_{n}(t), y_{n}(t)\right) \leq\left|y_{n}(t)\right|_{L^{4}}\left|\nabla y_{n}(t)\right|\left|z_{n}(t)\right|_{\mathbb{L}^{4}} \leq\left|y_{n}(t)\right|^{1 / 2}\left|\nabla y_{n}(t)\right|^{3 / 2}\left|z_{n}(t)\right|_{\mathbb{L}^{4}} \\
& \leq \frac{\nu}{16}\left|\nabla y_{n}(t)\right|^{2}+\frac{3 \cdot 12^{2}}{\nu^{3}}\left|z_{n}(t)\right|_{L^{4}}^{4}\left|y_{n}(t)\right|^{2}, \\
& b\left(v(t), \hat{z}_{n}(t), y_{n}(t)\right) \leq|v(t)|_{L^{4}}\left|\nabla y_{n}(t)\right|\left|\hat{z}_{n}(t)\right|_{\mathbb{L}^{4}} \\
& \leq|v(t)|^{1 / 2}|\nabla v(t)|^{1 / 2}\left|\nabla y_{n}(t)\right|\left|\hat{z}_{n}(t)\right|_{\mathbb{L}^{4}} \\
& \leq \frac{\nu}{16}\left|\nabla y_{n}(t)\right|^{2}+\frac{4}{\nu}|v(t)||\nabla v(t)|\left|\hat{z}_{n}(t)\right|_{\mathbb{L}^{4}}^{2}, \\
& b\left(\hat{z}_{n}(t), v_{n}(t), y_{n}(t)\right) \leq\left|\hat{z}_{n}(t)\right|_{\mathbb{L}^{4}}\left|\nabla y_{n}(t)\right|\left|v_{n}(t)\right|^{1 / 2}\left|\nabla v_{n}(t)\right|^{1 / 2} \\
& \leq \frac{\nu}{16}\left|\nabla y_{n}(t)\right|^{2}+\frac{4}{\nu}\left|v_{n}(t)\right|\left|\nabla v_{n}(t)\right|\left|\hat{z}_{n}(t)\right|_{\mathbb{L}^{4}}^{2}, \\
& b\left(\hat{z}_{n}(t), z_{n}(t), y_{n}(t)\right) \leq\left|\hat{z}_{n}(t)\right|_{\mathbb{L}^{4}}\left|z_{n}(t)\right|_{\mathbb{L}^{4}}\left|\nabla y_{n}(t)\right| \\
& \leq \frac{\nu}{16}\left|\nabla y_{n}(t)\right|^{2}+\frac{4}{\nu}\left|z_{n}(t)\right|_{\mathbb{L}^{4}}^{2}\left|\hat{z}_{n}(t)\right|_{\mathbb{L}^{4}}^{2},
\end{aligned}
$$




$$
\begin{aligned}
b\left(z(t), \hat{z}_{n}(t), y_{n}(t)\right) & \leq\left|\hat{z}_{n}(t)\right|_{\mathbb{L}^{4}}|z(t)|_{\mathbb{L}^{4}}\left|\nabla y_{n}(t)\right| \\
& \leq \frac{\nu}{16}\left|\nabla y_{n}(t)\right|^{2}+\frac{4}{\nu}|z(t)|_{\mathbb{L}^{4}}^{2}\left|\hat{z}_{n}(t)\right|_{\mathbb{L}^{4}}^{2}, \\
\alpha\left(\hat{z}_{n}(t), y_{n}(t)\right) & \leq \alpha\left|y_{n}(t)\right|_{\mathrm{V}}\left|\hat{z}_{n}(t)\right|_{\mathrm{V}^{\prime}} \\
& \leq \frac{\nu}{16}\left|\nabla y_{n}(t)\right|^{2}+\frac{\nu}{16}\left|y_{n}(t)\right|^{2}+\frac{4 \alpha^{2}}{\nu}\left|\hat{z}_{n}(t)\right|_{\mathrm{V}^{\prime}}^{2}, \\
\left(\hat{f}_{n}(t), y_{n}(t)\right) & \leq\left|y_{n}(t)\right|_{\mathrm{V}}\left|\hat{f}_{n}(t)\right|_{\mathrm{V}^{\prime}} \\
& \leq \frac{\nu}{16}\left|\nabla y_{n}(t)\right|^{2}+\frac{\nu}{16}\left|\nabla y_{n}(t)\right|^{2}+\frac{4}{\nu}\left|\hat{f}_{n}(t)\right|_{\mathrm{V}^{\prime}}^{2} .
\end{aligned}
$$

Hence we have, weakly on $(0, T)$,

$$
\begin{aligned}
\frac{d}{d t}\left|y_{n}(t)\right|^{2}+\nu\left|\nabla y_{n}(t)\right|^{2} & \leq \frac{8}{\nu}\left|\nabla v_{n}(t)\right|^{2}\left|y_{n}(t)\right|^{2}+\frac{6 \cdot 12^{2}}{\nu^{3}}\left|z_{n}(t)\right|_{L^{4}}^{4}\left|y_{n}(t)\right|^{2} \\
& +\frac{8}{\nu}|v(t)||\nabla v(t)|\left|\hat{z}_{n}(t)\right|_{\mathbb{L}^{4}}^{2}+\frac{8}{\nu}\left|v_{n}(t)\right|\left|\nabla v_{n}(t)\right|\left|\hat{z}_{n}(t)\right|_{\mathbb{L}^{4}}^{2} \\
& +\frac{8}{\nu}|z(t)|_{\mathbb{L}^{4}}^{2}\left|\hat{z}_{n}(t)\right|_{\mathbb{L}^{4}}^{2}+\frac{8}{\nu}\left|z_{n}(t)\right|_{\mathbb{L}^{4}}^{2}\left|\hat{z}_{n}(t)\right|_{\mathbb{L}^{4}}^{2} \\
& +\frac{8 \alpha^{2}}{\nu}\left|\hat{z}_{n}(t)\right|_{\mathrm{V}^{\prime}}^{2}+\frac{8}{\nu}\left|\hat{f}_{n}(t)\right|_{\mathrm{V}^{\prime}}^{2}
\end{aligned}
$$

By integrating the above inequality from 0 to $t, t \in[0, T]$, we get that

$$
\begin{aligned}
\left|y_{n}(t)\right|^{2}+\nu \int_{0}^{t}\left|\nabla y_{n}(s)\right|^{2} d s & \leq\left|y_{n}(0)\right|^{2} \\
& +\int_{0}^{t} \alpha_{n}(s)\left|y_{n}(s)\right|^{2} d s+\frac{8}{\nu} \int_{0}^{t} \beta_{n}(s) d s, t \in[0, T],
\end{aligned}
$$

where

$$
\begin{aligned}
\alpha_{n}(s) & =\frac{8}{\nu}\left|\nabla v_{n}(s)\right|^{2}+\frac{\nu}{8} \frac{6 \cdot 12^{2}}{\nu^{3}}\left|z_{n}(s)\right|_{L^{4}}^{4}, \quad s \in[0, T] \\
\beta_{n}(s) & =|v(s)||\nabla v(s)|\left|\hat{z}_{n}(s)\right|_{\mathbb{L}^{4}}^{2}+\left|v_{n}(s)\right|\left|\nabla v_{n}(s)\right|\left|\hat{z}_{n}(s)\right|_{\mathbb{L}^{4}}^{2}+|z(s)|_{\mathbb{L}^{4}}^{2}\left|\hat{z}_{n}(s)\right|_{\mathbb{L}^{4}}^{2} \\
& +\left|z_{n}(s)\right|_{\mathbb{L}^{4}}^{2}\left|\hat{z}_{n}(s)\right|_{\mathbb{L}^{4}}^{2}+\alpha^{2}\left|\hat{z}_{n}(s)\right|_{\mathrm{V}^{\prime}}^{2}+\left|\hat{f}_{n}(s)\right|_{\mathrm{V}^{\prime}}^{2}, \quad s \in[0, T] .
\end{aligned}
$$

Then by the Gronwall inequality,

$$
\left|y_{n}(t)\right|^{2} \leq\left(\left|y_{n}(0)\right|^{2}+\frac{8}{\nu} \int_{0}^{t} \beta_{n}(s) d s\right) e^{\int_{0}^{t} \alpha_{n}(s) d s} .
$$

On the other hand let us next observe that

$$
\begin{aligned}
\int_{0}^{T} \beta_{n}(s) d s= & \int_{0}^{T}\left[|v(s)||\nabla v(s)|\left|\hat{z}_{n}(s)\right|_{\mathbb{L}^{4}}^{2}+\left|v_{n}(s)\right|\left|\nabla v_{n}(s)\right|\left|\hat{z}_{n}(s)\right|_{\mathbb{L}^{4}}^{2}\right. \\
& \left.+\left|\hat{z}_{n}(s)\right|_{\mathbb{L}^{4}}^{2}|z(s)|_{\mathbb{L}^{4}}^{2}+\left|z_{n}(s)\right|_{\mathbb{L}^{4}}^{2}\left|\hat{z}_{n}(s)\right|_{\mathbb{L}^{4}}^{2}+\alpha^{2}\left|\hat{z}_{n}(s)\right|_{\mathrm{V}^{\prime}}^{2}+\left|\hat{f}_{n}(s)\right|_{\mathrm{V}^{\prime}}^{2}\right] d s \\
\leq\left[\left.|v|\right|_{L^{\infty}(0, T ; \mathrm{H})}|v|_{L^{2}(0, T ; \mathrm{V})}+\left|v_{n}\right|_{L^{\infty}(0, T ; \mathrm{H})}\left|v_{n}\right|_{L^{2}(0, T ; \mathrm{V})}\right. & \\
\quad & \left.+\left|z_{n}\right|_{L^{4}\left(0, T ; \mathbb{L}^{4}\right)}^{2}+\left|z_{n}\right|_{L^{4}\left(0, T ; \mathbb{L}^{4}\right)}^{2}\right]\left|\hat{z}_{n}\right|_{L^{4}\left(0, T ; \mathbb{L}^{4}\right)}^{2} \\
& +\alpha^{2}\left|\hat{z}_{n}\right|_{L^{2}\left(0, T ; \mathrm{V}^{\prime}\right)}^{2}+\left|\hat{f}_{n}\right|_{L^{2}\left(0, T ; \mathrm{V}^{\prime}\right)}^{2} .
\end{aligned}
$$


Therefore, $\int_{0}^{T} \beta_{n}(s) d s \rightarrow 0$, as $n \rightarrow \infty$. Since also $\left|y_{n}(0)\right| \rightarrow 0$, as $n \rightarrow \infty$ and for some constant $C<\infty$ and all $n \in \mathbb{N}$,

$$
\begin{aligned}
\int_{0}^{T} \alpha_{n}(s) d s & =\int_{0}^{T}\left(\frac{8}{\nu}\left|\nabla v_{n}(s)\right|^{2}+\frac{\nu}{8}+\frac{6 \cdot 12^{2}}{\nu^{3}}\left|z_{n}(s)\right|_{L^{4}}^{4}\right) d s \\
& \leq \frac{8}{\nu}\left|v_{n}(s)\right|_{L^{2}(0, T ; \mathrm{V})}^{2}+\frac{6 \cdot 12^{2}}{\nu^{3}}\left|z_{n}(s)\right|_{L^{4}\left(0, T ; \mathbb{L}^{4}\right)}^{4}+T \frac{\nu}{8} \leq C,
\end{aligned}
$$

we infer that $y_{n}(t) \rightarrow 0$ in $\mathrm{H}$ as $n \rightarrow \infty$, uniformly in $t \in[0, T]$. In other words,

$$
v\left(\cdot, z_{n}\right) x_{n} \rightarrow v(\cdot, z) x \quad \text { in } C([0, T] ; \mathrm{H}) .
$$

From inequality (5.19) we also have

$$
\begin{aligned}
\nu \int_{0}^{T}\left|\nabla y_{n}(s)\right|^{2} d s & \leq\left|y_{n}(0)\right|^{2}+\int_{0}^{T} \alpha_{n}(s)\left|y_{n}(s)\right|^{2} d s+\frac{8}{\nu} \int_{0}^{T} \beta_{n}(s) d s \\
& \leq\left|y_{n}(0)\right|^{2}+\int_{0}^{T} \alpha_{n}(s) d s \sup _{s \in[0, T]}\left|y_{n}(s)\right|^{2}+\frac{8}{\nu} \int_{0}^{T} \beta_{n}(s) d s .
\end{aligned}
$$

Hence, $\int_{0}^{T}\left|\nabla y_{n}(s)\right|^{2} d s \rightarrow 0$ as $n \rightarrow \infty$ and therefore,

$$
v\left(\cdot, z_{n}\right) x_{n} \rightarrow v(\cdot, z) x \quad \text { in } L^{2}(0, T ; \mathrm{V}),
$$

which concludes the proof.

The existence and uniqueness result proved earlier works naturally when the initial time is no longer 0 , but any $s \in \mathbb{R}$. For the sake of completeness, we state the corresponding result.

Theorem 5.5. Under the above assumptions, if $\alpha \geq 0, z \in \mathbb{L}_{\text {loc }}^{4}\left([s, \infty) ; \mathbb{L}^{2}(D)\right) \cap$ $L_{\mathrm{loc}}^{2}\left([s, \infty) ; \mathrm{V}^{\prime}\right), x \in \mathrm{H}$ and $f \in \mathrm{V}^{\prime}$, there exists a unique solution $v(\cdot, s ; z, x)$ of equation (4.19), such that it belongs to $L_{\mathrm{loc}}^{2}([s, \infty) ; \mathrm{V}) \cap C([s, \infty) ; \mathrm{H})$ and $v(s, s ; z, x)=x$.

\section{RDS generated by the 2D stochastic Navier Stokes equations}

After all the hard work in the previous section is completed, we are able to define an RDS $\varphi$ corresponding to problem (4.16) in H. But first we need to take a "good" model for our probability space.

6.1. Wiener process. The following is our standing assumption.

Assumption A.1. $\mathrm{K} \subset \mathrm{H} \cap \mathbb{L}^{4}$ is a Hilbert space such that for some $\delta \in(0,1 / 2)$,

$$
\mathrm{A}^{-\delta}: \mathrm{K} \rightarrow \mathrm{H} \cap \mathbb{L}^{4} \text { is } \gamma \text {-radonifying. }
$$

Remark 6.1. (a) Condition (6.1) means that $A^{-\delta}: \mathrm{K} \rightarrow \mathrm{H}$ is Hilbert Schmidt and $A^{-\delta}: \mathrm{K} \rightarrow \mathrm{H} \cap \mathbb{L}^{4}$ is $\gamma$-radonifying. Let us recall (see e.g. [7]) that for real separable Hilbert and resp. Banach spaces $\mathrm{K}$ and $\mathrm{X}$, a bounded linear operator $\mathrm{L}: \mathrm{K} \rightarrow \mathrm{X}$ is called $\gamma$-radonifying iff $\mathrm{L}\left(\gamma_{\mathrm{K}}\right)$ is $\sigma$-additive. Here $\gamma_{\mathrm{K}}$ is the canonical cylindrical finitely additive set-valued function (also called a Gaussian distribution) on K. If $\mathrm{L}: \mathrm{K} \rightarrow \mathrm{X}$ is $\gamma$-radonifying, then $\mathrm{L}\left(\gamma_{\mathrm{K}}\right)$ has a unique extension to a $\sigma$-additive Borel probability measure $\nu_{\mathrm{L}}$ on $\mathrm{X}$. It can be shown that $\nu_{\mathrm{L}}$ is a centered Gaussian measure on X whose Reproducing Kernel Hilbert Space (RKHS)1 (also called the Cameron Martin space) is K. In particular, in spirit of the celebrated L Gross's

\footnotetext{
${ }^{1}$ RKHS of a centered Gaussian measure $\mu$ on a separable Banach space X (see [20], p. 40) is a (unique) Hilbert space $\left(\mathrm{G},|\cdot|_{\mathrm{G}}\right)$ such that $\mathrm{G} \hookrightarrow \mathrm{X}$ continuously and for each $\varphi \in \mathrm{X}^{*}$ the random variable $\varphi$ on probability space $(\mathrm{X}, \mu)$ is normal with mean 0 and variance $|\varphi| \mathrm{G}^{2}$.
} 
paper [28, the triple $\left(\mathrm{K}, \mathrm{X}, \nu_{\mathrm{L}}\right)$ is an Abstract Wiener Space (AWS). By $R(\mathrm{~K}, \mathrm{X})$ we denote the Banach space of $\gamma$-radonifying operators from $\mathrm{K}$ to $\mathrm{X}$ with norm

$$
\|\mathrm{L}\|_{R(\mathrm{~K}, \mathrm{X})}:=\left\{\int_{X}|x|^{2} d \nu_{\mathrm{L}}(x)\right\}^{\frac{1}{2}}, \quad \mathrm{~L} \in \mathrm{R}(\mathrm{K}, \mathrm{X}) .
$$

It is now well established (see e.g. Neidhardt [36]) that $\mathrm{R}(\mathrm{K}, \mathrm{X})$ with norm (6.2) is a separable Banach space and that the set $\mathcal{L}_{\text {fin }}(H, X)$ of bounded linear operators $L: H \rightarrow X$ with finite-dimensional range is a dense subspace of $\mathrm{R}(\mathrm{K}, \mathrm{X})$. It is also well known (see e.g. Baxendale [3]) that $R(K, X)$ is an operator ideal, i.e. if $\mathrm{L}_{2} \in \mathrm{R}(\mathrm{K}, \mathrm{X})$ and $\mathrm{L}_{1} \in \mathcal{L}(\mathrm{G}, \mathrm{K})$ and $L_{3} \in \mathcal{L}(\mathrm{X}, \mathrm{Y})$ (where $\mathrm{G}$, resp. $\mathrm{Y}$, is a separable Hilbert, resp. Banach, space), then $L_{3} L_{2} L_{1} \in \mathrm{R}(\mathrm{G}, \mathrm{Y})$ and $\left\|L_{3} L_{2} L_{1}\right\|_{\mathrm{R}(\mathrm{G}, \mathrm{Y})} \leq$ $C\left|L_{3}\right|_{\mathcal{L}(\mathrm{X}, \mathrm{Y})}|| \mathrm{L}_{2} \|_{\mathrm{R}(\mathrm{K}, \mathrm{X})}\left|L_{1}\right|_{\mathcal{L}(\mathrm{G}, \mathrm{K})}$ for some constant $C$ independent of $L_{1}, L_{2}$ and $L_{3}$.

(b) Note that because $A^{-s}$ is a bounded operator in $\mathrm{H} \cap \mathbb{L}^{4}$ for $s>0$, if condition (6.1) is satisfied for some $\delta_{1}$, then it is also satisfied for any $\delta_{2} \geq \delta_{1}$.

(c) It may be worth mentioning Theorem 2.3 from [12. Let $p \in(1, \infty)$ be fixed. Let $\left(\mathcal{O}_{i}, \mathcal{F}_{i}, \nu_{i}\right), i=1,2$, be $\sigma$-finite measure spaces. A bounded linear operator $K: L^{2}\left(\mathcal{O}_{1}\right) \rightarrow L^{p}\left(\mathcal{O}_{2}\right)$ is $\gamma$-radonifying iff there exists a measurable function $\kappa:$ $\mathcal{O}_{1} \times \mathcal{O}_{2} \rightarrow \mathbb{R}$ such that $\int_{\mathcal{O}_{2}}\left[\int_{\mathcal{O}_{1}}\left|\kappa\left(x_{1}, x_{2}\right)\right|^{2} d \nu_{1}\left(x_{1}\right)\right]^{p / 2} d \nu_{2}\left(x_{2}\right)<\infty$, and for all $\nu$-almost all $x_{2} \in \mathcal{O}_{2},(K(f))\left(x_{2}\right)=\int_{\mathcal{O}_{1}} \kappa\left(x_{1}, x_{2}\right) f\left(x_{1}\right) d \nu_{1}\left(x_{1}\right), f \in L^{2}\left(\mathcal{O}_{1}\right)$. It follows that if $\mathcal{O}$ is a bounded domain, then $\mathrm{A}^{-s}: \mathrm{H} \rightarrow \mathbb{L}^{p}(D)$ is $\gamma$-radonifying iff $\int_{D}\left[\sum_{j} \lambda_{j}^{-2 s}\left|e_{j}(x)\right|^{2}\right]^{p / 2} d x<\infty$, where $e_{j}$ is an ONB of $\mathrm{H}$ and $A e_{j}=\lambda_{j} e_{j}, j \in \mathbb{N}$. Hence, if $D$ is a 2-dimensional torus, then $\mathrm{A}^{-s}$ is $\gamma$-radonifying iff $s>\frac{1}{2}$. In other words, with $K=D\left(A^{s}\right)$, the embedding $K \hookrightarrow \mathrm{H} \cap \mathbb{L}^{4}$ is $\gamma$-radonifying iff $s>1 / 2$. Therefore, Assumption A.1 is satisfied for any $\delta>0$. Indeed, condition (6.1) holds iff the operator $\mathrm{A}^{-(s+\delta)}: \mathrm{H} \rightarrow \mathrm{H} \cap \mathbb{L}^{4}$ is $\gamma$-radonifying. However, for domains of more complicated geometrical structure the situation is more refined; see e.g. [42.

(d) We require in Assumption A.1 that $\delta<1 / 2$ because we want (see subsection 6.3) the corresponding Ornstein-Uhlenbeck process to take values in $H \cap \mathbb{L}^{4}$.

Let us denote $\mathrm{X}=\mathrm{H} \cap \mathbb{L}^{4}$ and let $\mathrm{E}$ be the completion of $A^{-\delta}(\mathrm{X})$ with respect to the image norm $|x|_{\mathrm{E}}=\left|A^{-\delta} x\right|_{\mathrm{X}}, x \in \mathrm{X}$. It is well known that $\mathrm{E}$ is a separable Banach space. For $\xi \in(0,1 / 2)$ we set

$$
C_{1 / 2}^{\xi}(\mathbb{R}, \mathrm{E}):=\left\{\omega \in C(\mathbb{R}, \mathrm{E}): \omega(0)=0, \sup _{t, s \in \mathbb{R}} \frac{|\omega(t)-\omega(s)|_{\mathrm{E}}}{|t-s|^{\xi}(1+|t|+|s|)^{1 / 2}}<\infty\right\} .
$$

It is easy to prove that $C_{1 / 2}^{\xi}(\mathbb{R}, \mathrm{E})$ endowed with a norm

$$
\|\omega\|_{C_{1 / 2}^{\xi}(\mathbb{R}, \mathrm{E})}=\sup _{t, s \in \mathbb{R}} \frac{|\omega(t)-\omega(s)|_{\mathrm{E}}}{|t-s|^{\xi}(1+|t|+|s|)^{1 / 2}}
$$

is a nonseparable Banach space. However, the closure of $\left\{\omega \in C_{0}^{\infty}(\mathbb{R}, \mathrm{E}): \omega(0)=0\right\}$ in $C_{1 / 2}^{\xi}(\mathbb{R}, \mathrm{E})$, denoted by $\Omega(\xi, \mathrm{E})$, is a separable Banach space.

For $\xi=0$ we have a similar definition. Let us denote by $C_{1 / 2}(\mathbb{R}, \mathrm{X})$ the space of all continuous functions $\omega: \mathbb{R} \rightarrow \mathrm{X}$ of linear growth condition, i.e. for some $C=C(\omega)>0$,

$$
|\omega(t)| \leq C\left(1+|t|^{1 / 2}\right), \quad t \in \mathbb{R}
$$


The space $C_{1 / 2}(\mathbb{R}, \mathrm{E})$ endowed with a norm

$$
\|\omega\|_{C_{1 / 2}(\mathbb{R}, \mathrm{E})}=\sup _{t \in \mathbb{R}} \frac{|\omega(t)|_{\mathrm{E}}}{1+|t|^{1 / 2}}
$$

is a separable Banach space.

We denote by $\mathcal{F}$ the Borel $\sigma$-algebra on $\Omega(\xi, \mathrm{E})$. One can show by methods from [5] (but see also 29] for a similar problem in the one-dimensional case) that for $\xi \in(0,1 / 2)$, there exists a Borel probability measure $\mathbb{P}$ on $\Omega(\xi, \mathrm{E})$ such that the canonical process $w_{t}, t \in \mathbb{R}$, defined by

$$
w_{t}(\omega):=\omega(t), \quad \omega \in \Omega(\xi, \mathrm{E}),
$$

is a two-sided Wiener process such that the Cameron-Martin (or Reproducing Kernel Hilbert) space of the Gaussian measure $\mathcal{L}\left(w_{1}\right)$ on $\mathrm{E}$ is equal to K. For $t \in \mathbb{R}$, let $\mathcal{F}_{t}:=\sigma\left\{w_{s}: s \leq t\right\}$. Since for each $t \in \mathbb{R}$ the map $z \circ i_{t}: \mathrm{E}^{*} \rightarrow L^{2}\left(\Omega(\xi, \mathrm{E}), \mathcal{F}_{t}, \mathbb{P}\right)$, where $i_{t}: \Omega(\xi, \mathrm{E}) \ni \gamma \mapsto \gamma(t) \in \mathrm{E}$, satisfies $\mathbb{E}\left|z \circ i_{t}\right|^{2}=t|z|_{\mathrm{K}}^{2}$, there exists a unique extension of $z \circ i_{t}$ to a bounded linear map $W_{t}: \mathrm{K} \rightarrow L^{2}\left(\Omega(\xi, \mathrm{E}), \mathcal{F}_{t}, \mathbb{P}\right)$. Moreover, the family $\left(W_{t}\right)_{t \in \mathbb{R}}$ is an H-cylindrical Wiener process on a filtered probability space $\left(\Omega(\xi, \mathrm{E}),\left(\mathfrak{F}_{t}\right)_{t \in \mathbb{R}}, \mathbb{P}\right)$ in the sense of e.g. [11.

On the space $C_{1 / 2}(\mathbb{R}, \mathrm{X})$ we consider a flow $\vartheta=\left(\vartheta_{t}\right)_{t \in \mathbb{R}}$ defined by

$$
\vartheta_{t} \omega(\cdot)=\omega(\cdot+t)-\omega(t), \quad \omega \in \Omega, t \in \mathbb{R} .
$$

This flow keeps the spaces $C_{1 / 2}^{\xi}(\mathbb{R}, \mathrm{E})$ and $\Omega(\xi, \mathrm{E})$ invariant, and we will often denote by $\vartheta_{t}$ the restriction of $\vartheta_{t}$ to one of these spaces.

It is obvious that for each $t \in \mathbb{R}, \vartheta_{t}$ preserves $\mathbb{P}$. In order to define an OrnsteinUhlenbeck process we need some analytic preliminaries. These are presented in the next subsection.

\subsection{Analytic preliminaries.}

Proposition 6.2. Assume that $A$ is a generator of an analytic semigroup $\left\{e^{-t A}\right\}_{t \geq 0}$ on a separable Banach space X, such that for some $C>0$ and $\gamma>0$

$$
\left\|A^{1+\delta} e^{-t A}\right\|_{\mathcal{L}(\mathrm{X}, \mathrm{X})} \leq C t^{-1-\delta} e^{-\gamma t}, \quad t \geq 0 .
$$

For $\xi \in\left(\delta, \frac{1}{2}\right)$ and $\tilde{\omega} \in C_{1 / 2}^{\xi}(\mathbb{R}, \mathrm{X})$ we define

$$
\hat{z}(t)=\hat{z}(\tilde{\omega})(t)=\int_{-\infty}^{t} A^{1+\delta} e^{-(t-r) A}(\tilde{\omega}(t)-\tilde{\omega}(r)) d r, t \in \mathbb{R} .
$$

If $t \in \mathbb{R}$, then $\hat{z}(t)$ is a well-defined element of $\mathrm{X}$ and the mapping

$$
C_{1 / 2}^{\xi}(\mathbb{R}, \mathrm{X}) \ni \tilde{\omega} \mapsto \hat{z}(t) \in \mathrm{X}
$$

is continuous. Moreover, the map $\hat{z}: C_{1 / 2}^{\xi}(\mathbb{R}, \mathrm{X}) \rightarrow C_{1 / 2}(\mathbb{R}, \mathrm{X})$ is well defined, linear and bounded. In particular, there exists a constant $C_{2}<\infty$ such that for any $\tilde{\omega} \in C_{1 / 2}^{\xi}(\mathbb{R}, \mathrm{X})$

$$
|\hat{z}(\tilde{\omega})(t)| \leq C_{2}\left(1+|t|^{1 / 2}\right)\|\tilde{\omega}\|, \quad t \in \mathbb{R} .
$$

Remark 6.3. Since $\Omega(\xi, \mathrm{X})$ is a closed subspace of $C_{1 / 2}^{\xi}(\mathbb{R}, \mathrm{X})$, Proposition 6.2 is also valid with the latter space replaced by the former. 
Proof. Part I. Let $\tilde{\omega} \in C_{1 / 2}^{\xi}(\mathbb{R}, \mathrm{X})$ and $t \in \mathbb{R}$. In order to prove that $\hat{z}(t)$ is a well-defined element of $\mathrm{X}$, we need to show that

$$
\int_{-\infty}^{t}\left|A^{1+\delta} e^{-(t-r) A}(\tilde{\omega}(t)-\tilde{\omega}(r))\right| d r<\infty
$$

By the change of variables (with $s=t-r$ ), from the definition of $\hat{z}(\tilde{\omega})(t)$ we see that

$$
\begin{aligned}
\int_{-\infty}^{t}\left|A^{1+\delta} e^{-(t-r) A}(\tilde{\omega}(t)-\tilde{\omega}(r))\right| d r & =\int_{0}^{\infty}\left|A^{1+\delta} e^{-s A}(\tilde{\omega}(t)-\tilde{\omega}(t-s))\right| d s, \\
\hat{z}(\tilde{\omega})(t) & =\int_{0}^{\infty} A^{1+\delta} e^{-s A}(\tilde{\omega}(t)-\tilde{\omega}(t-s)) d s .
\end{aligned}
$$

From (6.5) we have

$$
\int_{0}^{\infty}\left|A^{1+\delta} e^{-s A}(\tilde{\omega}(t)-\tilde{\omega}(t-s))\right| d s \leq C \int_{0}^{\infty} \frac{e^{-\gamma s}}{s^{1+\delta}}|\tilde{\omega}(t)-\tilde{\omega}(t-s)| d s .
$$

Since $\tilde{\omega} \in C_{1 / 2}^{\xi}(\mathbb{R}, \mathrm{X})$,

$$
\begin{aligned}
|\tilde{\omega}(t)-\tilde{\omega}(t-s)| & \leq|s|^{\xi}(1+|t|+|t-s|)^{1 / 2} \sup _{t, s \in \mathbb{R}} \frac{|\tilde{\omega}(t)-\tilde{\omega}(t-s)|}{|s|^{\xi}(1+|t|+|t-s|)^{1 / 2}} \\
& =\|\tilde{\omega}\|_{C_{1 / 2}^{\xi}(\mathbb{R}, \mathrm{X})}|s|^{\xi}(1+|t|+|t-s|)^{1 / 2} \\
& \leq\|\tilde{\omega}\|_{C_{1 / 2}^{\xi}(\mathbb{R}, \mathrm{X})}|s|^{\xi}\left(1+|2 t|^{1 / 2}+|s|^{1 / 2}\right), \quad s, t \in \mathbb{R} .
\end{aligned}
$$

Then, with $C_{1}=\int_{0}^{\infty} \frac{e^{-\gamma s}}{s^{1+\delta-\xi}}\left(1+s^{1 / 2}\right) d s$ and $C_{2}=\sqrt{2} \int_{0}^{\infty} \frac{e^{-\gamma s}}{s^{1+\delta-\xi}} d s$, we have

$$
\begin{aligned}
\int_{0}^{\infty} \frac{e^{-\gamma s}}{s^{1+\delta}}|\tilde{\omega}(t)-\tilde{\omega}(t-s)| d s & \leq\|\tilde{\omega}\|_{C_{1 / 2}^{\xi}(\mathbb{R}, \mathrm{X})} \int_{0}^{\infty} \frac{e^{-\gamma s}}{s^{1+\delta}} s^{\xi}\left(1+|2 t|^{1 / 2}+s^{1 / 2}\right) d s \\
& \leq\|\tilde{\omega}\|_{C_{1 / 2}^{\xi}(\mathbb{R}, \mathrm{X})}\left(C_{1}+C_{2}|t|^{1 / 2}\right)<\infty .
\end{aligned}
$$

Hence, by (6.9) and (6.11), and $\delta<\xi$, i.e. $1+\delta-\xi<1$, we infer that $\hat{z}(t)$ is a welldefined element of X. Moreover, we proved inequality (6.7) with $C=\max \left\{C_{1}, C_{2}\right\}$.

Part II. Continuity with respect to $t$. For any $t, t_{0} \in \mathbb{R}$ we have

$$
\begin{aligned}
\left|\hat{z}(t)-\hat{z}\left(t_{0}\right)\right| & \leq \int_{0}^{\infty}\left|A^{1+\delta} e^{-s A}\left(\tilde{\omega}(t)-\tilde{\omega}(t-s)-\tilde{\omega}\left(t_{0}\right)+\tilde{\omega}\left(t_{0}-s\right)\right)\right| d s \\
& \leq C \int_{0}^{\infty} \frac{e^{-\gamma s}}{s^{1+\delta}}\left|\tilde{\omega}(t)-\tilde{\omega}(t-s)-\tilde{\omega}\left(t_{0}\right)+\tilde{\omega}\left(t_{0}-s\right)\right| d s \\
& =I I\left(t, t_{0}\right) .
\end{aligned}
$$

It is enough to show that for fixed $t_{0} \in \mathbb{R}$ and a sequence $\left(t_{n}\right)_{n \in \mathbb{N}}$ such that $t_{n} \rightarrow t_{0}$,

$$
I\left(t_{n}, t_{0}\right) \rightarrow 0, \quad \text { as } n \rightarrow \infty, \text { for } k=1,2 .
$$

Since $\tilde{\omega}$ is a continuous $\mathrm{X}$-valued function, the integrand converges to 0 for all $s \in(0, \infty)$. Therefore, in view of the Lebesgue Dominated Convergence Theorem in order to show (6.13), it is enough to find an integrable function $g:[0, \infty) \rightarrow \mathbb{R}^{+}$ such that for all $s>0$ and all $n \in \mathbb{N}, \frac{e^{-\gamma s}}{s^{1+\delta}}\left|\tilde{\omega}(t)-\tilde{\omega}(t-s)-\tilde{\omega}\left(t_{0}\right)+\tilde{\omega}\left(t_{0}-s\right)\right| \leq g(s)$. 
Since $t_{n} \rightarrow t_{0}$, there exists $K>0$ such that $1+2\left|t_{n}\right| \leq K$ for all $n \in \mathbb{N}$. Therefore, in view of (6.10), for all $n \in \mathbb{N}$,

$$
\left|\tilde{\omega}\left(t_{n}\right)-\tilde{\omega}\left(t_{n}-s\right)\right| d s \leq\|\tilde{\omega}\|_{C_{1 / 2}^{\xi}(\mathbb{R}, \mathrm{X})}|s|^{\xi}\left(K^{1 / 2}+s^{1 / 2}\right) \frac{e^{-\gamma s}}{|s|^{1+\delta}} .
$$

If we define $g(s)=C\|\tilde{\omega}\|_{C_{1 / 2}^{\xi}(\mathbb{R}, \mathrm{X})} s^{\xi}\left(K^{1 / 2}+s^{1 / 2}\right) \frac{e^{-\gamma s}}{|s|^{1+\delta}}, s>0$, we only need to show integrability of the function $g$. This will follow provided we can show that

$$
\int_{0}^{1} \frac{e^{-\gamma s}}{s^{1+\delta-\xi}}\left(1+s^{1 / 2}\right) d s<\infty \quad \text { and } \quad \int_{1}^{\infty} \frac{e^{-\gamma s}}{s^{1+\delta-\xi}}\left(1+s^{1 / 2}\right) d s<\infty .
$$

The first of these two inequalities is true because $\delta<\xi$, and the second is true because $\gamma>0$. This concludes the proof of the second part.

Part III. The map $\hat{z}$. From Parts I and II of the proof we infer that for any $\tilde{\omega} \in C_{1 / 2}^{\xi}(\mathbb{R}, \mathrm{X})$ the function $\hat{z}(\tilde{\omega})$ belongs to $C_{1 / 2}(\mathbb{R}, \mathrm{X})$. Hence, the map $\hat{z}$ : $C_{1 / 2}^{\xi}(\mathbb{R}, \mathrm{X}) \rightarrow C_{1 / 2}(\mathbb{R}, \mathrm{X})$ is well defined. Obviously, it is a linear map. From the observation at the end of Part I, inequality (6.7) is satisfied, and hence $\hat{z}$ is also a bounded map. This concludes proof of the whole proposition.

We have the following direct consequence of the previous result.

Corollary 6.4. Under the assumptions of Proposition 6.2, for any $t \in \mathbb{R}$ and any $-\infty<a<b<\infty$, the maps

$$
\begin{array}{lll}
C_{1 / 2}^{\xi}(\mathbb{R}, \mathrm{X}) \ni \tilde{\omega} & \mapsto \hat{z}(\tilde{\omega})(t) \in \mathrm{X}, \\
C_{1 / 2}^{\xi}(\mathbb{R}, \mathrm{X}) \ni \tilde{\omega} & \mapsto \hat{z}(\tilde{\omega}) \in L^{4}(a, b ; \mathrm{X})
\end{array}
$$

are continuous.

Remark 6.5. It is clear that in the second part of Corollary 6.4 the exponent 4 can be replaced by any $q \in[1, \infty]$.

As in the case of Proposition 6.2, Corollary 6.4 is valid with the space $C_{1 / 2}^{\xi}(\mathbb{R}, \mathrm{X})$ replaced by $\Omega(\xi, \mathrm{X})$.

Theorem 6.6. Under the assumptions of Proposition 6.2 , for any $\omega \in C_{1 / 2}^{\xi}(\mathbb{R}, \mathrm{X})$,

$$
\hat{z}\left(\vartheta_{s} \omega\right)(t)=\hat{z}(\omega)(t+s), \quad t, s \in \mathbb{R} .
$$

In particular, for any $\omega \in \Omega$ and all $t, s \in \mathbb{R}, \hat{z}\left(\vartheta_{s} \omega\right)(0)=\hat{z}(\omega)(s)$.

Proof. Since $\left(\vartheta_{s} w\right)(r)=\omega(r+s)-\omega(s), r \in \mathbb{R}$, we have

$$
\begin{aligned}
\hat{z}\left(\vartheta_{s} \omega\right)(t) & =\int_{-\infty}^{t} A^{1+\delta} e^{-(t-r) A}\left[\vartheta_{s} \omega(t)-\vartheta_{s} \omega(r)\right] d r \\
& =A^{1+\delta} \int_{-\infty}^{t} e^{-(t-r) A}[\omega(t+s)-\omega(r+s)] d r \\
& =A^{1+\delta} \int_{-\infty}^{t+s} e^{-\left(t+s-r^{\prime}\right) A}\left[\omega(t+s)-\omega\left(r^{\prime}\right)\right] d r^{\prime}=\hat{z}(\omega)(t+s) .
\end{aligned}
$$


Remark 6.7. For $\zeta \in C_{1 / 2}(\mathbb{R}, \mathrm{X})$ we put

$$
\left(\tau_{s} \zeta\right)=\zeta(t+s), \quad t, s \in \mathbb{R} .
$$

Thus, $\tau_{s}$ is a map from $C_{1 / 2}(\mathbb{R}, \mathrm{X})$ into itself. Moreover, it is a linear and bounded map, and the family $\left(\tau_{s}\right)_{s \in \mathbb{R}}$ is a strongly continuous group of bounded linear operators on $C_{1 / 2}(\mathbb{R}, \mathrm{X})$.

Using the notation introduced above, Theorem 6.6 can be rewritten in the following way.

Corollary 6.8. For $s \in \mathbb{R}$ one has

$$
\tau_{s} \circ \hat{z}=\hat{z} \circ \vartheta_{s} .
$$

In other words, for all $s \in \mathbb{R}$ and $\omega \in C_{1 / 2}^{\xi}(\mathbb{R}, \mathrm{X}), \tau_{s}(\hat{z}(\omega))=\hat{z}\left(\vartheta_{s}(\omega)\right)$.

6.3. Ornstein-Uhlenbeck process. In this and the following subsections we are concerned with the linear evolutional Stokes equations. Hence in particular, $\mathrm{H}$ is the Hilbert space introduced in section 4 and $A$ is the linear operator defined therein (called the Stokes operator). The spaces X and E have been defined in subsection 6.1 .

Note that in this framework, for any $\nu>0$ and $\alpha \geq 0,(\nu A+\alpha I)^{\delta}: \mathrm{E} \rightarrow \mathrm{X}$ is a bounded linear map and so is the induced map $\Omega(\xi, \mathrm{E}) \ni \omega \mapsto(\nu A+\alpha I)^{\delta} \omega \in$ $\Omega(\xi, \mathrm{X})$.

For $\delta$ as in Assumption A.1, $\alpha \geq 0, \nu>0, \xi \in(\delta, 1 / 2)$ and $\omega \in C_{1 / 2}^{\xi}(\mathbb{R}, \mathrm{E})$ (so that $\left.(\nu A+\alpha I)^{-\delta} \omega \in C_{1 / 2}^{\xi}(\mathbb{R}, \mathrm{X})\right)$, we define $z_{\alpha}(\omega):=\hat{z}\left((\nu A+\alpha I)^{-\delta} \omega\right) \in$ $C_{1 / 2}(\mathbb{R}, \mathrm{X})$, i.e. for any $t \geq 0$,

$$
z_{\alpha}(\omega)(t):=\int_{-\infty}^{t}(\nu A+\alpha I)^{1+\delta} e^{-(t-r)(\nu A+\alpha I)}
$$

$$
\begin{gathered}
{\left[(\nu A+\alpha I)^{-\delta} \omega(t)-(\nu A+\alpha I)^{-\delta} \omega(r)\right] d r} \\
=\int_{-\infty}^{t}(\nu A+\alpha I)^{1+\delta} e^{-(t-r)(\nu A+\alpha I)}\left((\nu A+\alpha I)^{-\delta} \theta_{r} \omega\right)(t-r) d r .
\end{gathered}
$$

It can be shown, by invoking integration by parts, that if $\omega \in C_{0}^{\infty}(\mathbb{R}, \mathrm{E})$ is such that $\omega(0)=0$, then $z_{\alpha}$ satisfies the following equation:

$$
\frac{d z_{\alpha}(t)}{d t}+(\nu A+\alpha I) z_{\alpha}=\dot{\omega}(t), \quad t \in \mathbb{R} .
$$

Therefore, from the definition of the space $\Omega(\xi, \mathrm{E})$, we have

Corollary 6.9. If $\alpha, \beta \geq 0$, then the difference $z_{\alpha}-z_{\beta}$ is a solution to

$$
\frac{d\left(z_{\alpha}-z_{\beta}\right)(t)}{d t}+\nu A\left(z_{\alpha}-z_{\beta}\right)(t)=\left(-\alpha z_{\alpha}+\beta z_{\beta}\right)(t), \quad t \in \mathbb{R} .
$$

Analogously to our definition (6.4) of the Wiener process $w(t), t \in \mathbb{R}$, we can view the formula (6.16) as a definition of a process $z_{\alpha}(t), t \in \mathbb{R}$, on the probability space $(\Omega(\xi, \mathrm{E}), \mathcal{F}, \mathbb{P})$. Equation (6.17) suggests that this process is an Ornstein-Uhlenbeck process. In fact we have the following result.

Proposition 6.10. The process $z_{\alpha}(t), t \in \mathbb{R}$, is a stationary Ornstein-Uhlenbeck process. It is a solution of the equation

$$
d z_{\alpha}(t)+(\nu A+\alpha I) z_{\alpha} d t=d w(t), \quad t \in \mathbb{R}
$$


i.e. for all $t \in \mathbb{R}$, a.s.

$$
z_{\alpha}(t)=\int_{-\infty}^{t} e^{-(t-s)(\nu A+\alpha I)} d w(s)
$$

where the integral is the Itô integral on the M-type 2 Banach space $X$ in the sense of $[6]$.

In particular, for some $C=C(\mathrm{X})$,

$$
\begin{aligned}
\mathbb{E}\left|z_{\alpha}(t)\right|_{\mathrm{X}}^{2} & =\mathbb{E}\left|\int_{-\infty}^{t} e^{(\nu \mathrm{A}+\alpha I)(s-t)} d w(s)\right|_{\mathrm{X}}^{2} \leq C \int_{-\infty}^{t}\left\|e^{(\nu \mathrm{A}+\alpha)(s-t)}\right\|_{R(\mathrm{~K}, \mathrm{X})}^{2} d s \\
& =C \int_{0}^{\infty} e^{-2 \alpha s}\left\|e^{-\nu s \mathrm{~A}}\right\|_{R(\mathrm{~K}, \mathrm{X})}^{2} d s .
\end{aligned}
$$

Moreover, $\mathbb{E}\left|z_{\alpha}(t)\right|_{\mathrm{X}}^{2}$ tends to 0 as $\alpha \rightarrow \infty$.

Proof. Stationarity of the process $z_{\alpha}$ follows from the following version of Theorem 6.6 .

$$
z_{\alpha}\left(\vartheta_{s} \omega\right)(t)=z_{\alpha}(\omega)(t+s), \quad \omega \in C_{1 / 2}^{\xi}(\mathbb{R}, \mathrm{X}), t, s \in \mathbb{R} .
$$

The equality (6.19) follows by finite-dimensional approximation, and inequality (6.20) follows from 6. Finally, the last statement follows from (6.20) by applying the Lebesgue Dominated Convergence Theorem.

Remark 6.11. Because $e^{-s A}=A^{\delta} e^{-s A} A^{-\delta}$, it follows by Neidhardt 36 that

$$
\left\|e^{-s A}\right\|_{R(\mathrm{~K}, \mathrm{X})} \leq\left|A^{\delta} e^{-s A}\right|_{\mathcal{L}(\mathrm{X}, \mathrm{X})}\left\|A^{-\delta}\right\|_{R(\mathrm{~K}, \mathrm{X})} .
$$

Therefore, since $\left|A^{\delta} e^{-s A}\right|_{\mathcal{L}(\mathrm{X}, \mathrm{X})} \leq C s^{-\delta} e^{-\gamma s}$, for some $C, \gamma>0$ and all $s>0$, we infer that if Assumption A.1 is satisfied, then for $\delta<\frac{1}{2}$ the integral on the RHS of (6.20) is finite.

Note also that, since $z_{\alpha}(t)$ is a Gaussian random vector, for each $p \geq 2$, there exists a constant $C_{p}>0$, such that $\mathbb{E}\left|z_{\alpha}(t)\right|_{\mathrm{X}}^{p} \leq C_{p}\left(\mathbb{E}\left|z_{\alpha}(t)\right|_{\mathrm{X}}^{2}\right)^{p / 2}$.

Remark 6.12. Our definition of the O-U process was motivated by 24]. A similar idea can also be found in an unpublished work [32].

Since by Proposition 6.10, $z_{\alpha}(t), t \in \mathbb{R}$, is a stationary and ergodic X-valued process, by the Strong Law of Large Numbers (see Da Prato and Zabczyk [21] for a similar argument),

$$
\lim _{k \rightarrow \infty} \frac{1}{k} \int_{-k}^{0}\left|z_{\alpha}(s)\right|_{\mathrm{X}}^{2} d s=\mathbb{E}\left|z_{\alpha}(0)\right|_{\mathrm{X}}^{2}, \quad \text { a.s. }
$$

Denote by $\Omega_{\alpha}(\xi, \mathrm{E})$ the set of those $\omega \in \Omega(\xi, \mathrm{E})$ for which the equality (6.21) holds true. As mentioned above, $\Omega_{\alpha}(\xi, \mathrm{E})$ is of full measure. Moreover, it follows from Corollary 6.8 that this set is invariant with respect to the flow $\vartheta$, i.e. for all $\alpha \geq 0$ and all $t \in \mathbb{R}, \vartheta_{t}\left(\Omega_{\alpha}(\xi, \mathrm{E})\right) \subset \Omega_{\alpha}(\xi, \mathrm{E})$. Therefore, the same is true for a set

$$
\hat{\Omega}(\xi, \mathrm{E})=\bigcap_{n=0}^{\infty} \Omega_{n}(\xi, \mathrm{E}) .
$$

It follows that as a model for a metric DS we can take either a quadruple $(\Omega(\xi, \mathrm{E}), \mathcal{F}$, $\mathbb{P}, \vartheta)$ or a quadruple $(\hat{\Omega}(\xi, \mathrm{E}), \hat{\mathcal{F}}, \hat{\mathbb{P}}, \hat{\vartheta})$, where $\hat{\mathcal{F}}, \hat{\mathbb{P}}$ and $\hat{\vartheta}$ are respectively the natural restrictions of $\mathcal{F}, \mathbb{P}$ and $\vartheta$ to $\hat{\Omega}(\xi, \mathrm{E})$. We will see why there is need of 
the latter in section 8 in the proof of Proposition 8.1 Summing up, we have the following.

Proposition 6.13. The quadruples $(\Omega(\xi, \mathrm{E}), \mathcal{F}, \mathbb{P}, \vartheta)$ and $(\hat{\Omega}(\xi, \mathrm{E}), \hat{\mathcal{F}}, \hat{\mathbb{P}}, \hat{\vartheta})$ are both metric DSs. For each $\omega \in \hat{\Omega}(\xi, \mathrm{E})$ the limit in (6.21) exists and

$$
\int_{\hat{\Omega}(\xi, \mathrm{E})}\left|z_{n}(\omega)(0)\right|^{2} d \hat{\mathbb{P}}(\omega) \rightarrow 0, \quad \text { as } n \rightarrow \infty .
$$

6.4. RDS. Let us recall that we suppose that Assumption A.1 is satisfied and that $\delta$ has the property stated there. We also take a fixed viscosity $\nu>0$ and some parameter $\alpha \geq 0$ which we will vary in the following sections. We also fix $\xi \in(\delta, 1 / 2)$ and put $\Omega:=\Omega(\xi, \mathrm{E})$.

Definition 6.14. We define a map $\varphi=\varphi_{\alpha}: \mathbb{R}_{+} \times \Omega \times \mathrm{H} \rightarrow \mathrm{H}$ by

$$
(t, \omega, x) \mapsto v(t, z(\omega))(x-z(\omega)(0))+z(\omega)(t),
$$

where for simplicity of notation we put $z=z_{\alpha}$.

Because $z(\omega) \in C_{1 / 2}(\mathbb{R}, \mathrm{X}), z(\omega)(0)$ is a well-defined element of $\mathrm{H}$ and hence $\varphi$ is well defined. Furthermore, we have the main result of this section.

Theorem 6.15. $(\varphi, \vartheta)$ is an $R D S$.

Proof. All properties with the exception of the cocycle one of an RDS follow from Theorem 4.6. Hence we only need to show that for any $x \in \mathrm{H}$,

$$
\varphi(t+s, \omega) x=\varphi\left(t, \vartheta_{s} \omega\right) \varphi(s, \omega) x, \quad t, s \in \mathbb{R}_{+} .
$$

From the definition of $\varphi$ and noting that by Theorem 6.6 $z(\omega)(s)=z\left(\vartheta_{s} \omega\right)(0)$, $s \in \mathbb{R}$, we have for $t, s \in \mathbb{R}_{+}$,

$$
\begin{aligned}
\varphi(t+s, \omega) x & =v(t+s, z(\omega)(t+s))(x-z(\omega)(0))+z(\omega)(t+s), \\
\varphi\left(t, \vartheta_{s} \omega\right) \varphi(s, \omega) x & =v\left(t, z\left(\vartheta_{s} \omega\right)(t)\right)(v(s, z(\omega)(t))(x-z(\omega)(0))+z(\omega)(s) \\
& \left.-z\left(\vartheta_{s} \omega\right)(0)\right)+z\left(\vartheta_{s} \omega\right)(t) \\
& =v\left(t, z\left(\vartheta_{s} \omega\right)(t)\right)(v(s, z(\omega)(s))(x-z(\omega)(0)))+z\left(\vartheta_{s} \omega\right)(t) .
\end{aligned}
$$

Therefore, in view of Theorem 6.6, in order to prove (6.23), we only need to prove, for any $t, s \in \mathbb{R}_{+}$,

$$
\begin{aligned}
& v(t+s, z(\omega)(t+s))(x-z(\omega)(0)) \\
& =v\left(t, z\left(\vartheta_{s} \omega\right)(t)\right)(v(s, z(\omega)(s))(x-z(\omega)(0))) .
\end{aligned}
$$

Let us fix $s \in \mathbb{R}_{+}$and define two functions $v_{1}$ and $v_{2}$ by

$$
\begin{aligned}
& v_{1}(t)=v(t+s, z(\omega)(t+s))(x-z(\omega)(0)), \quad t \in \mathbb{R}_{+}, \\
& v_{2}(t)=v\left(t, z\left(\vartheta_{s} \omega\right)(t)\right)(v(s, z(\omega)(s))(x-z(\omega)(0))), \quad t \in \mathbb{R}_{+} .
\end{aligned}
$$

Because $v\left(0, z\left(\vartheta_{s} \omega\right)(0)\right)\left(x-z\left(\vartheta_{s} \omega\right)(0)\right)=x-z\left(\vartheta_{s} \omega\right)(0)$, we infer that

$$
\begin{aligned}
v_{1}(0) & =v(s, z(\omega)(s))(x-z(\omega)(0)) \\
& =v\left(0, z\left(\vartheta_{s} \omega\right)(0)\right)(v(s, z(\omega)(s))(x-z(\omega)(0)))=v_{2}(0) .
\end{aligned}
$$

Since $\mathbb{R}_{+} \ni t \mapsto v(t, z(\omega))$ is a solution to problem (4.19)-(4.20) and

$$
v_{1}^{\prime}(t)=\frac{d v(\cdot, z(\omega))}{d t}(t+s)
$$


we have

$$
\begin{array}{r}
v_{1}^{\prime}(t)=-\nu A v(t+s, z(\omega)(t+s))-B(v(t+s, z(\omega)(t+s))+z(\omega)(t+s)) \\
+\alpha z(\omega)(t+s)+f \\
=-\nu A v_{1}(t ; z(\omega))-B\left(v_{1}(t ; z(\omega))+z(\omega)(t+s)\right)+\alpha z(\omega)(t+s)+f
\end{array}
$$

On the other hand we have

$$
\begin{aligned}
& \frac{d v\left(t, z\left(\vartheta_{s} \omega\right)(t)\right)}{d t} \\
= & -\nu A v\left(t, z\left(\vartheta_{s} \omega\right)(t)\right)-B\left(v\left(t, z\left(\vartheta_{s} \omega\right)(t)\right)+z\left(\vartheta_{s} \omega\right)(t)\right)+\alpha z\left(\vartheta_{s} \omega\right)(t)+f .
\end{aligned}
$$

Thus $v_{1}$ solves the problem

$$
\left\{\begin{array}{l}
\frac{d v_{1}(t)}{d t}=-\nu A v_{1}(t)-B\left(v_{1}(t)+z(\omega)(t+s)\right)+\alpha z(\omega)(t+s)+f \\
v_{1}(0)=v(z(\omega))(s)(x-z(\omega)(0))
\end{array}\right.
$$

and $v_{2}$ solves the problem

$$
\left\{\begin{array}{l}
\frac{d v_{2}(t)}{d t}=-\nu A v_{2}(t)-B\left(v_{2}(t)+z\left(\vartheta_{s} \omega\right)(t)\right)+\alpha z\left(\vartheta_{s} \omega\right)(t)+f, \\
v_{2}(0)=v(z(\omega))(s)(x-z(\omega)(0)) .
\end{array}\right.
$$

By Theorem 6.6, $z\left(\vartheta_{s} \omega\right)(t)=z(\omega)(t+s)$ for $t \geq 0$. Hence both $v_{1}$ and $v_{2}$ are solutions to problem (4.19) - (4.20) with the same initial data

$$
v(s, z(\omega)(s))(x-z(\omega)(0))
$$

at initial time 0 . Therefore, by the uniqueness of solutions to problem (4.19)-(4.20), we infer that $v_{1}(t)=v_{2}(t), t \in \mathbb{R}_{+}$.

Finally, since $s$ is arbitrary in $\mathbb{R}_{+}$, we have proved (6.24), which concludes our result.

Since in the definition of $\varphi$ we have used a fixed $\alpha \geq 0$, we should rather have denoted it by $\varphi_{\alpha}$. On the other hand, as $\alpha$ does not enter problem (4.16), and hence it is an auxiliary parameter, we should clarify the issue of whether $\varphi_{\alpha}$ depends on $\alpha$. We have proved earlier in Corollary 6.9 that this is the case for a linear problem (i.e. for the O-U process). Below we give an affirmative answer to the full stochastic NSEs.

Proposition 6.16. If $\alpha, \beta \geq 0$, then $\varphi_{\alpha}=\varphi_{\beta}$.

Proof. Let us fix $x \in \mathrm{H}$. We need to show that

$$
v^{\alpha}(t)+z_{\alpha}(t)=v^{\beta}(t)+z_{\beta}(t), \quad t \geq 0,
$$

where $z_{\alpha}$ is defined by (6.16) and $v^{\alpha}$ is a solution to problem (4.19)-(4.20) with initial data $\left(x-z_{\alpha}(0)\right)$. From (4.19)-(4.20) we infer that $v^{\alpha}(0)-v^{\beta}(0)=-z_{\alpha}(0)+$ $z_{\beta}(0)$ and

$$
\begin{aligned}
& \frac{d\left(v^{\alpha}(t)-v^{\beta}(t)\right)}{d t}=-\nu A\left(v^{\alpha}(t)-v^{\beta}(t)\right)+\left(\alpha z_{\alpha}(t)-\beta z_{\beta}(t)\right) \\
&+\left[B\left(v^{\alpha}(t)+z_{\alpha}(t)\right)-B\left(v^{\beta}(t)+z_{\beta}(t)\right)\right], t \geq 0
\end{aligned}
$$


Adding the above equation to (6.18), we get

$$
\frac{d\left(u^{\alpha}(t)-u^{\beta}(t)\right)}{d t}=-\nu A\left(u^{\alpha}(t)-v^{\beta}(t)\right)+\left[B\left(u^{\alpha}(t)\right)-B\left(v^{\beta}(t)\right)\right], t \geq 0,
$$

where $u^{\alpha}(t)=v^{\alpha}(t)+z_{\alpha}(t), v^{\beta}(t)=v^{\beta}(t)+z_{\beta}(t), t \geq 0$ and $u^{\alpha}(0)-u^{\beta}(0)=0$.

Next applying Lemma 5.1 to the function $u^{\alpha}-u^{\beta}$ and using inequality (4.13), we get, weakly on $(0, \infty)$,

$$
\begin{aligned}
& \frac{1}{2} \frac{d}{d t}\left|u^{\alpha}(t)-u^{\beta}(t)\right|^{2}+\nu\left\|u^{\alpha}(t)-u^{\beta}(t)\right\|^{2} \\
\leq & \left|u^{\alpha}(t)\right|_{\mathbb{L}^{4}}\left|u^{\alpha}(t)-u^{\beta}(t)\right|_{\mathbb{L}^{2}}^{1 / 2}\left\|u^{\alpha}(t)-u^{\beta}(t)\right\|^{3 / 2} \\
\leq & \frac{\nu}{2}\left\|u^{\alpha}(t)-u^{\beta}(t)\right\|^{2}+\frac{2}{\nu^{3}}\left|u^{\alpha}(t)\right|_{\mathbb{L}^{4}}^{4}\left|u^{\alpha}(t)-u^{\beta}(t)\right|^{2}, t \geq 0 .
\end{aligned}
$$

Hence,

$$
\frac{d}{d t}\left|u^{\alpha}(t)-u^{\beta}(t)\right|^{2} \leq \frac{4}{\nu^{3}}\left|u^{\alpha}(t)\right|_{\mathbb{L}^{4}}^{4}\left|u^{\alpha}(t)-u^{\beta}(t)\right|^{2}, t \geq 0 .
$$

Since $+\frac{4}{\nu^{3}} \int_{0}^{t}\left|u^{\alpha}(\tau)\right|_{\mathbb{L}^{4}}^{4} d \tau<\infty$ and $\left|u^{\alpha}(0)-u^{\beta}(0)\right|^{2}=0$, by applying the Gronwall Lemma we infer that $\left|u^{\alpha}(t)-u^{\beta}(t)\right|^{2}=0$, for all $t \geq 0$. This implies that $v^{\alpha}(t)+z_{\alpha}(t)=v^{\beta}(t)+z_{\beta}(t), t \geq 0$, which concludes the proof.

Now we are ready to present the definition and some fundamental properties of the solution to problem (4.16) with initial data $u_{0} \in \mathrm{H}$ at the initial time $s \in \mathbb{R}$.

Definition 6.17. Suppose that Assumption A.1 is satisfied. If $u_{s} \in \mathrm{H}, s \in \mathbb{R}, f \in$ $\mathrm{V}^{\prime}$ and $W_{t}, t \in \mathbb{R}$ is a two-sided Wiener process introduced after (6.4) such that the Cameron-Martin (or Reproducing Kernel Hilbert) Space of the Gaussian measure $\mathcal{L}\left(w_{1}\right)$ is equal to $\mathrm{K}$. A process $u(t), t \geq 0$, with trajectories in $C([s, \infty) ; \mathrm{H}) \cap$ $L_{\text {loc }}^{2}([s, \infty) ; \mathrm{V}) \cap L_{\text {loc }}^{4}\left([s, \infty) ; \mathbb{L}^{4}(D)\right)$ is a solution to problem (4.16) iff $u(s)=u_{s}$ and for any $\phi \in \mathrm{V}, t>s$,

$$
\begin{aligned}
(u(t), \phi) & =(u(s), \phi)-\nu \int_{s}^{t}(u(r), \mathrm{A} \phi) d r-\int_{s}^{t} b(u(r), u(r), \phi) d r \\
& +\int_{s}^{t}(f, \phi) d r+\int_{s}^{t}\left\langle\phi, d W_{r}\right\rangle .
\end{aligned}
$$

The following result follows easily by applying Lemma 4.2 and Remark 4.3 (ii).

Proposition 6.18. In the framework of Definition 6.17, suppose that $u(t)=$ $z_{\alpha}(t)+v_{\alpha}(t), t \geq s$, where $v_{\alpha}$ is the unique solution to problem (4.19) (4.20) with initial data $u_{0}-z_{\alpha}(s)$ at time $s$. If the process $u(t), t \geq s$, has trajectories in $C([s, \infty) ; \mathrm{H}) \cap L_{\text {loc }}^{2}([s, \infty) ; \mathrm{V}) \cap L_{\text {loc }}^{4}\left([s, \infty) ; \mathbb{L}^{4}(D)\right)$, then it is a solution to problem (4.16). Vice-versa, if a process $u(t), t \geq s$, with trajectories in $C([s, \infty) ; \mathrm{H}) \cap L_{\mathrm{loc}}^{2}([s, \infty) ; \mathrm{V}) \cap L_{\mathrm{loc}}^{4}\left([s, \infty) ; \mathbb{L}^{4}(D)\right)$ is a solution to problem (4.16), then for any $\alpha \geq 0$, a process $v_{\alpha}(t), t \geq s$, defined by $z_{\alpha}(t)=u(t)-v_{\alpha}(t), t \geq s$, is a solution to (4.19) on $[s, \infty)$.

Our previous results yield the existence and the uniqueness of solutions to problem (4.16) as well as its continuous dependence on the data (in particular on the initial value $u_{0}$ and the force $f$ ). Moreover, if we define, for $x \in \mathrm{H}, \omega \in \Omega$, and $t \geq s$,

$$
u\left(t, s ; \omega, u_{0}\right):=\varphi\left(t-s ; \vartheta_{s} \omega\right) u_{0}=v\left(t, s ; \omega, u_{0}-z(s)\right)+z(t),
$$


then for each $s \in \mathbb{R}$ and each $u_{0} \in \mathrm{H}$, the process $u(t), t \geq s$, is a solution to problem (4.16).

\section{The WEAK CONTINUity OF THE RDS GENERATED BY STOCHASTIC NSES}

In this section, $v\left(\cdot, v_{0}\right)$ for $v_{0} \in \mathrm{H}$ denotes the unique solution to the initial value problem (4.19) -(4.20), with a deterministic function $z \in L_{\mathrm{loc}}^{4}\left(\mathbb{R}^{+}, \mathbb{L}^{4}(D)\right) \cap$ $L_{\mathrm{loc}}^{2}\left(\mathbb{R}^{+}, \mathrm{V}^{\prime}\right)$.

Lemma 7.1. If $T>0$, then the map

$$
\mathrm{H} \ni x \mapsto v(\cdot, x) \in L^{2}([0, T] ; \mathrm{V})
$$

is continuous in the weak topologies.

Lemma 7.2. If $T>0$, then the maps

$$
\mathrm{H} \ni x \mapsto v(t, x) \in \mathrm{H}, t \in[0, T]
$$

are continuous in the weak topologies. More precisely, if $x_{n} \rightarrow x$ weakly in $\mathrm{H}$, then for any $\phi \in \mathrm{H},\left(v\left(\cdot, x_{n}\right), \phi\right) \rightarrow(v(\cdot, x), \phi)$ uniformly on $[0, T]$, as $n \rightarrow \infty$.

Proof of Lemma 7.1. Suppose that $\left\{x_{n}\right\}_{n}$ is an H-valued sequence that is weakly convergent to $x \in \mathrm{H}$. Let $v_{n}=v\left(\cdot, x_{n}\right)$ and $v=v(\cdot, x)$. Since the sequence $\left\{x_{n}\right\}_{n}$ is bounded in $\mathrm{H}$, by Proposition 5.4 we infer that for $\gamma \in\left(0, \frac{1}{2}\right]$,

the sequence $\left\{v_{n}\right\}_{n}$ is bounded in $L^{\infty}(0, T ; \mathrm{H}) \cap L^{2}(0, T ; \mathrm{V}) \cap \mathcal{H}^{\gamma, 2}(0, T ; \mathrm{V}, \mathrm{H})$.

Hence without loss of generality we may assume that there exists a $\tilde{v} \in L^{2}(0, T ; \mathrm{V}) \cap$ $L^{\infty}(0, T ; \mathrm{H})$, such that, as $n \rightarrow \infty$,

$$
v_{n} \rightarrow \tilde{v} \text { weak-star in } L^{\infty}(0, T ; \mathrm{H}) \text { and weakly in } L^{2}(0, T ; \mathrm{V}) .
$$

Moreover, with $D_{r}=D \cap\left\{x \in \mathbb{R}^{2}:|x|<r\right\}$, we have that for any $r>0$,

the sequence $\left\{\left.v_{n}\right|_{(0, T) \times D_{r}}\right\}_{n}$ is bounded in $\mathcal{H}^{\gamma, 2}\left(0, T ; H^{1}\left(D_{r}\right), \mathbb{L}^{2}\left(D_{r}\right)\right)$.

As before, by the compactness theorem (40], Theorem III.2.2) and by using the diagonal procedure, without loss of generality we may assume that for any $r>0$,

$$
\left.\left.v_{n}\right|_{(0, T) \times D_{r}} \rightarrow \tilde{v}\right|_{(0, T) \times D_{r}} \quad \text { strongly in } L^{2}\left(0, T ; \mathbb{L}^{2}\left(D_{r}\right)\right) .
$$

It is then standard to prove that $\tilde{v}$ is a solution of (4.19) with $\tilde{v}(0)=x$ and hence by the uniqueness of the solutions infer that $\tilde{v}=v$. Next, because the weak topology on a bounded subset of a separable Hilbert space is metrizable, we deduce that the whole sequence $\left\{v_{n}\right\}_{n}$ converges to $v$ weakly in $L^{2}(0, T ; \mathrm{V})$.

Proof of Lemma 7.2, Suppose that $\left\{x_{n}\right\}_{n}$ is an H-valued sequence, weakly convergent to some $x \in \mathrm{H}$. Let $v_{n}=v\left(\cdot, x_{n}\right)$ and $v=v(\cdot, x)$. By the proof of the previous lemma, (7.5) holds true. Take $\phi \in \mathcal{V}$. Then, by (7.5), for almost every $t \in[0, T]$, $\left(v_{n}(t), \phi\right) \rightarrow(v(t), \phi)$. Moreover, since by (7.3) the sequence $\left\{v_{n}\right\}_{n}$ is bounded in $L^{\infty}(0, T ; \mathrm{H}),\left\{\left(v_{n}(\cdot), \phi\right)\right\}_{n}$ is uniformly bounded on $[0, T]$.

On the other hand, in view of Proposition 5.4 $\left\|v_{n}^{\prime}\right\|_{L^{2}\left(0, T ; \mathrm{V}^{\prime}\right)} \leq C$ for some $C>0$ and all $n \in \mathbb{N}$. Therefore by the Cauchy-Schwartz inequality, for all $0 \leq t \leq t+a \leq$ $T$ and $n \in \mathbb{N}$, we have

$$
\left|\left(v_{n}(t+a)-v_{n}(t), \phi\right)\right| \leq\left|\left(\int_{t}^{t+a} v_{n}^{\prime}(s) d s, \phi\right)\right| \leq C\|\phi\| \sqrt{a} .
$$


This shows that the sequence $\left\{\left(v_{n}(\cdot), \phi\right)\right\}_{n}$ is uniformly continuous on $[0, T]$. Hence, by the Arzela-Ascoli Theorem, there exists a subsequence $\left\{v_{n^{\prime}}\right\}$, such that $\left(v_{n^{\prime}}(\cdot), \phi\right)$ $\rightarrow(v(\cdot), \phi)$ uniformly on $[0, T]$. Next using the standard contradiction argument, we infer that

$$
\left(v_{n}(\cdot), \phi\right) \rightarrow(v(\cdot), \phi) \text { uniformly on }[0, T] .
$$

Since $\mathcal{V}$ is dense in $\mathrm{H}$, and $\sup _{n \in \mathbb{N}, t \in[0, T]}\left|v_{n}(t)\right|<\infty$, then for any $\phi \in \mathrm{H}$,

$$
\left(v_{n}(t), \phi\right) \rightarrow(v(t), \phi) \quad \text { weakly in } \mathrm{H} \quad \text { uniformly in } t \in[0, T],
$$

which finishes the proof of Lemma 7.2

\section{Asymptotic COMPACTNESS OF THE RDS GENERATED BY STOCHASTIC NSES}

In this section we assume that the domain $D$ is a Poincaré domain, i.e. such that the Poincaré inequality (4.2) holds true on $D$. Hence, in particular, the following inequalities are satisfied:

$$
\begin{gathered}
\|u\|^{2} \geq \lambda_{1}|u|^{2}, \quad \text { for all } u \in \mathrm{V}, \\
|\mathrm{A} u|^{2} \geq \lambda_{1}\|u\|^{2}, \quad \text { for all } u \in D(\mathrm{~A}) .
\end{gathered}
$$

Here we consider the $\operatorname{RDS} \varphi$ over the metric $\operatorname{DS}(\hat{\Omega}(\xi, \mathrm{E}), \hat{\mathcal{F}}, \hat{\mathbb{P}}, \hat{\vartheta})$; see Proposition 6.13. The main result in this section is the following result.

Proposition 8.1. The RDS $\varphi$ is asymptotically compact provided for any bounded set $B \subset \mathrm{H}$ there exists a closed and bounded random set $K(\omega)$ absorbing $B$.

Remark 8.2. It was pointed out to us by David Elworthy that our results can be related to a paper by S. G. Jones [31]. We will investigate this possible relationship in a future publication.

Let us recall that the $\operatorname{RDS} \varphi$ is independent of the auxiliary parameter $\alpha \in \mathbb{N}$. For reasons that will become clear in the course of the proof, we choose $\alpha$ such that $\mathbb{E}\left|z_{\alpha}(0)\right|_{\mathbb{L}^{4}}^{2} \leq \frac{\nu^{2} \lambda_{1}}{6 C^{2}}$, where $z_{\alpha}(t), t \in \mathbb{R}$, is the Ornstein-Uhlenbeck process from subsection 6.3. $C>0$ is a certain universal constant, $\lambda_{1}$ is the constant from (8.1) and $\nu>0$ is the viscosity. Such a choice is possible because of Proposition 6.13. For simplicity of notation we will denote the space $\hat{\Omega}(\xi, \mathrm{E})$ simply by $\Omega$ and the process $z_{\alpha}(t), t \in \mathbb{R}$, by $z(t), t \in \mathbb{R}$.

Proof. Suppose that $B \subset \mathrm{H}$ a bounded set, $\left(t_{n}\right)_{n=1}^{\infty}$ is an increasing sequence of positive numbers such that $t_{n} \rightarrow \infty$ and $\left(x_{n}\right)_{n}$ is a $B$-valued sequence. By our assumptions we can find a closed bounded random set $K(\omega)$ in $\mathrm{H}$ that absorbs $B$. We fix $\omega \in \Omega$.

Step I. Reduction. Since $K(\omega)$ absorbs $B$, for $n \in \mathbb{N}$ sufficiently large, $\varphi\left(t_{n}, \vartheta_{-t_{n}} \omega\right) B \subset K(\omega)$. Since $K(\omega)$ is closed and bounded, and hence weakly compact, without loss of generality we may assume that $\varphi\left(t_{n}, \vartheta_{-t_{n}} \omega\right) B \subset K(\omega)$ for all $n \in \mathbb{N}$ and, for some $y_{0} \in K(\omega)$,

$$
\varphi\left(t_{n}, \vartheta_{-t_{n}} \omega\right) x_{n} \rightarrow y_{0} \quad \text { weakly in } \mathrm{H} .
$$

Since $z(0) \in \mathrm{H}$, then

$$
\varphi\left(t_{n}, \vartheta_{-t_{n}} \omega\right) x_{n}-z(0) \rightarrow y_{0}-z(0) \quad \text { weakly in } \mathrm{H} .
$$


In particular,

$$
\left|y_{0}-z(0)\right| \leq \liminf _{n \rightarrow \infty}\left|\varphi\left(t_{n}, \vartheta_{-t_{n}} \omega\right) x_{n}-z(0)\right| .
$$

We claim that it is enough to prove that for some subsequence $\left\{n^{\prime}\right\} \subset \mathbb{N}$

$$
\left|y_{0}-z(0)\right| \geq \limsup _{n^{\prime} \rightarrow \infty}\left|\varphi\left(t_{n^{\prime}}, \vartheta_{-t_{n^{\prime}}} \omega\right) x_{n^{\prime}}-z(0)\right| .
$$

Indeed, since $\mathrm{H}$ is a Hilbert space, (8.4) in conjunction with (8.5) imply that

$$
\varphi\left(t_{n^{\prime}}, \vartheta_{-t_{n^{\prime}}} \omega\right) x_{n^{\prime}}-z(0) \rightarrow y_{0}-z(0) \text { strongly in } \mathrm{H}
$$

which easily implies that

$$
\varphi\left(t_{n^{\prime}}, \vartheta_{-t_{n^{\prime}}} \omega\right) x_{n^{\prime}} \rightarrow y_{0} \text { strongly in } \mathrm{H} .
$$

Summing up, in order to show that $\left\{\varphi\left(t_{n}, \vartheta_{-t_{n}} \omega\right) x_{n}\right\}_{n}$ is relatively compact in $\mathrm{H}$ we need to prove that (8.5) holds true.

Step II. Construction of a negative trajectory, i.e. a sequence $\left(y_{n}\right)_{n=-\infty}^{0}$ such that $y_{n} \in K\left(\theta_{n} \omega\right), n \in \mathbb{Z}^{-}$, and $y_{k}=\varphi\left(k-n, \theta_{n} \omega\right) y_{n}, n<k \leq 0$.

Since $K\left(\vartheta_{-1} \omega\right)$ absorbs $B$, there exists a constant $N_{1}(\omega) \in \mathbb{N}$, such that

$$
\left\{\varphi\left(-1+t_{n}, \vartheta_{1-t_{n}} \vartheta_{-1} \omega\right) x_{n}: n \geq N_{1}(\omega)\right\} \subset K\left(\vartheta_{-1} \omega\right) .
$$

Hence we can find a subsequence $\left\{n^{\prime}\right\} \subset \mathbb{N}$ and $y_{-1} \in K\left(\vartheta_{-1} \omega\right)$ such that

$$
\varphi\left(-1+t_{n^{\prime}}, \vartheta_{-t_{n^{\prime}}} \omega\right) x_{n^{\prime}} \rightarrow y_{1} \text { weakly in } \mathrm{H} .
$$

Let us observe that the cocycle property, with $t=1, s=t_{n^{\prime}}-1$, and $\omega$ being replaced by $\vartheta_{-t_{n^{\prime}}} \omega$, reads as follows: $\varphi\left(t_{n^{\prime}}, \vartheta_{-t_{n^{\prime}}} \omega\right)=\varphi\left(1, \vartheta_{-1} \omega\right) \varphi\left(-1+t_{n^{\prime}}, \vartheta_{-t_{n^{\prime}}} \omega\right)$. Hence, by Lemma 7.2. from (8.2) and (8.6) we infer that $\varphi\left(1, \vartheta_{-1} \omega\right) y_{1}=y_{0}$. By induction, for each $k=1,2, \ldots$, we can construct a subsequence $\left\{n^{(k)}\right\} \subset\left\{n^{(k-1)}\right\}$ and $y_{-k} \in$ $K\left(\vartheta_{-k} \omega\right)$, such that $\varphi\left(1, \vartheta_{-k} \omega\right) y_{-k}=y_{-k+1}$ and

$$
\varphi\left(-k+t_{n^{(k)}}, \vartheta_{-t_{n^{(k)}}} \omega\right) x_{n^{(k)}} \rightarrow y_{-k} \quad \text { weakly in } \mathrm{H}, \quad \text { as } n^{(k)} \rightarrow \infty .
$$

As above, the cocycle property with $t=k, s=t_{n^{(k)}}$ and $\omega$ being replaced by $\vartheta_{-t_{n(k)}} \omega$ yields

$$
\varphi\left(t_{n^{(k)}}, \vartheta_{-t_{n^{(k)}}} \omega\right)=\varphi\left(k, \vartheta_{-k} \omega\right) \varphi\left(t_{n^{(k)}}-k, \vartheta_{-t_{n}(k)} \omega\right), k \in \mathbb{N} .
$$

Hence, from (8.7) and by applying Lemma 7.1, we get

$$
\begin{aligned}
y_{0} & =\mathrm{w}-\lim _{n^{(k)} \rightarrow \infty} \varphi\left(t_{n^{(k)}}, \vartheta_{-t_{n}(k)} \omega\right) x_{n^{(k)}} \\
& =\mathrm{w}-\lim _{n^{(k)} \rightarrow \infty} \varphi\left(k, \vartheta_{-k} \omega\right) \varphi\left(t_{n^{(k)}}-k, \vartheta_{-t_{n^{(k)}}} \omega\right) x_{n^{(k)}} \\
& =\varphi\left(k, \vartheta_{-k} \omega\right)\left(\mathrm{w}-\lim _{n^{(k)} \rightarrow \infty} \varphi\left(t_{n^{(k)}}-k, \vartheta_{-t_{n}(k)} \omega\right) x_{n^{(k)}}\right)=\varphi\left(k, \vartheta_{-k} \omega\right) y_{-k},
\end{aligned}
$$

where $\mathrm{w}$ - lim denotes the limit in the weak topology on $\mathrm{H}$. The same proof yields a more general property:

$$
\varphi\left(j, \vartheta_{-k} \omega\right) y_{-k}=y_{-k+j} \text { if } 0 \leq j \leq k .
$$

Before we continue our proof, let us point out that (8.9) means precisely that $y_{0}=u\left(0,-k ; \omega, y_{-k}\right)$, where $u$ is defined by (6.26). 
Step III. Some inequalities.

Lemma 8.3. Suppose that $v$ is a solution to problem (4.19) on the time interval $[a, \infty)$ with $z \in L_{\mathrm{loc}}^{4}\left(\mathbb{R}^{+}, \mathbb{L}^{4}(D)\right) \cap L_{\mathrm{loc}}^{2}\left(\mathbb{R}^{+}, \mathrm{V}^{\prime}\right)$ and $\alpha \geq 0$. Denote $g(t)=\alpha z(t)-$ $B(z(t), z(t)), t \in[a, \infty)$. Then, for any $t \geq \tau \geq a$,

$$
\begin{aligned}
|v(t)|^{2} \leq & |v(\tau)|^{2} e^{-\nu \lambda_{1}(t-\tau)+\frac{3 C^{2}}{\nu} \int_{\tau}^{t}|z(s)|_{\mathbb{L}^{4}}^{2} d s} \\
& \frac{3}{\nu} \int_{\tau}^{t}\left\{|g(s)|_{\mathrm{V}^{\prime}}^{2}+|f|^{2}\right\} e^{-\nu \lambda_{1}(t-s)+\frac{3 C^{2}}{\nu} \int_{s}^{t}\left(|z(\zeta)|_{\mathbb{L}^{4}}^{2}\right) d \zeta} d s, \\
|v(t)|^{2}= & |v(\tau)|^{2} e^{-\nu \lambda_{1}(t-\tau)}+2 \int_{\tau}^{t} e^{-\nu \lambda_{1}(t-s)}(\langle B(v(s), z(s)), v(t)\rangle \\
& \left.+\langle g(s), v(s)\rangle+\langle f, v(s)\rangle-[v(s)]^{2}\right) d s .
\end{aligned}
$$

Proof. By Lemma 5.1, we have

$$
\begin{aligned}
\frac{1}{2} \frac{d}{d t}|v(t)|^{2}= & \langle-\nu \mathrm{A} v(t)-B(v(t))-B(z(t), v(t))+g(t)+f, v(t)\rangle \\
= & -\nu\langle\mathrm{A} v(t), v(t)\rangle+\langle B(v(t)), v(t)\rangle+\langle B(z(t), v(t)), v(t)\rangle \\
& \quad+\langle B(v(t), z(t)), v(t)\rangle+\langle g(t), v(t)\rangle+\langle f, v(t)\rangle \\
= & -\nu\|v(t)\|^{2}+b(v(t), z(t), v(t))+\langle g(t), v(t)\rangle+\langle f, v(t)\rangle .
\end{aligned}
$$

From (4.13), by using the Young inequality, we have

$$
\begin{aligned}
\mid b(v(t), z(t)), v(t)) \mid & \leq C|v(t)|_{\mathbb{L}^{2}}|\nabla v(t)|_{\mathbb{L}^{2}}|z(t)|_{\mathbb{L}^{4}} \\
& \leq \frac{\nu}{6}\|v(t)\|^{2}+\frac{3 C^{2}}{2 \nu}|v(t)|^{2}|z(t)|_{\mathbb{L}^{4}}^{2}, \\
|\langle g(t), v(t)\rangle+\langle f, v(t)\rangle| & \leq|g(t)|_{\mathrm{V}^{\prime}}\|v(t)\|+\left|f_{\mathrm{V}^{\prime}}\right|\|v(t)\| \\
& \leq \frac{\nu}{3}\|v(t)\|^{2}+\frac{3}{2 \nu}|g(t)|_{\mathrm{V}^{\prime}}^{2}+\frac{3}{2 \nu}|f|_{\mathrm{V}^{\prime}}^{2} .
\end{aligned}
$$

Hence from (8.12) and (8.1), we get

$$
\begin{aligned}
\frac{d}{d t}|v(t)|^{2} & \leq-\nu\|v(t)\|^{2}+\frac{3 C^{2}}{\nu}|z(t)|_{\mathbb{L}^{4}}^{2}|v(t)|^{2}+\frac{3}{\nu}|g(t)|_{\mathrm{V}^{\prime}}^{2}+\frac{3}{\nu}|f|_{\mathrm{V}^{\prime}}^{2} \\
& \leq\left(-\nu \lambda_{1}+\frac{3 C^{2}}{\nu}|z(t)|_{\mathbb{L}^{4}}^{2}\right)|v(t)|^{2}+\frac{3}{\nu}|g(t)|_{\mathrm{V}^{\prime}}^{2}+\frac{3}{\nu}|f|_{\mathrm{V}^{\prime}}^{2}
\end{aligned}
$$

Next, using the Gronwall Lemma, we arrive at (8.10).

As in [37, for any $u, v \in \mathrm{V}$, we define a new scalar product $[\cdot, \cdot]: \mathrm{V} \times \mathrm{V} \rightarrow \mathbb{R}$ by formula $[u, v]=\nu((u, v))-\nu \frac{\lambda_{1}}{2}(u, v)$. Clearly, $[\cdot, \cdot]$ is bilinear and symmetric. Moreover from (4.2), it is easy to prove that $[\cdot, \cdot]$ defines an inner product in $\mathrm{V}$ with norm $[\cdot]=[\cdot, \cdot]^{1 / 2}$, which is equivalent to the norm $\|\cdot\|$. By adding and subtracting $\nu \frac{\lambda_{1}}{2}|v(t)|^{2}$ from (8.12) we find that

$$
\begin{aligned}
\frac{d}{d t}|v(t)|^{2}+\nu & \lambda_{1}|v(t)|^{2}+2[v(t)]^{2} \\
& =2 b(v(t), z(t)), v(t))+2\langle g(t), v(t)\rangle+2\langle f, v(t)\rangle .
\end{aligned}
$$

Hence (8.11) follows by the variation of constants formula.

Step IV. Proof of (8.5). From now on, until explicitly stated, we fix $k \in \mathbb{N}$, and we will consider problem (4.16) on the time interval $[-k, 0]$. From (6.26) and (8.8), 
with $t=0$ and $s=-k$, we have

$$
\begin{aligned}
& \left|\varphi\left(t_{n^{(k)}}, \vartheta_{-t_{n^{(k)}}} \omega\right) x_{n^{(k)}}-z(0)\right|^{2} \\
& \quad=\left|\varphi\left(k, \vartheta_{-k} \omega\right) \varphi\left(t_{n^{(k)}}-k, \vartheta_{-t_{n^{(k)}}} \omega\right) x_{n^{(k)}}-z(0)\right|^{2} \\
& \quad=\left|v\left(0, \omega ;-k, \varphi\left(t_{n^{(k)}}-k, \vartheta_{-t_{n^{(k)}}} \omega\right) x_{n^{(k)}}-z(-k)\right)\right|^{2} .
\end{aligned}
$$

Let $v$ be the solution to (4.19) on $[-k, \infty)$ with $z=z_{\alpha}(\cdot, \omega)$ and the initial condition at time $\left.-k: v(-k)=\varphi\left(t_{n^{(k)}}-k, \vartheta_{-t_{n^{(k)}}} \omega\right) x_{n^{(k)}}-z(-k)\right)$. In other words,

$$
v(s)=v\left(s,-k ; \omega, \varphi\left(t_{n^{(k)}}-k, \vartheta_{-t_{n^{(k)}}} \omega\right) x_{n^{(k)}}-z(-k)\right), \quad s \geq-k .
$$

From (8.15) and (8.11) with $t=0$ and $\tau=-k$ we infer that

$$
\begin{aligned}
& \left|\varphi\left(t_{n^{(k)}}, \vartheta_{-t_{n^{(k)}}} \omega\right) x_{n^{(k)}}-z(0)\right|^{2}=e^{-\nu \lambda_{1} k}\left|\varphi\left(t_{n^{(k)}}-k, \vartheta_{-t_{n^{(k)}}} \omega\right) x_{n^{(k)}}-z(-k)\right|^{2} \\
& (8.16)+2 \int_{-k}^{0} e^{\nu \lambda_{1} s}\left(b(v(s), z(s), v(s))+\langle g(s), v(s)\rangle+\langle f, v(s)\rangle-[v(s)]^{2}\right) d s .
\end{aligned}
$$

It is enough to find a nonnegative function $h \in L^{1}(-\infty, 0)$ such that

$$
\limsup _{n^{(k)} \rightarrow \infty}\left|\varphi\left(t_{n^{(k)}}, \vartheta_{-t_{n^{(k)}}} \omega\right) x_{n^{(k)}}-z(0)\right|^{2} \leq \int_{-\infty}^{-k} h(s) d s+\left|y_{0}-z(0)\right|^{2} .
$$

For, if we define the diagonal process $\left(m_{j}\right)_{j=1}^{\infty}$ by $m_{j}=j^{(j)}, j \in \mathbb{N}$, then for each $k \in \mathbb{N}$, the sequence $\left(m_{j}\right)_{j=k}^{\infty}$ is a subsequence of the sequence $\left(n^{(k)}\right)$ and hence by (8.17), $\lim \sup _{j}\left|\varphi\left(t_{m_{j}}, \vartheta_{-t_{m_{j}}} \omega\right) x_{m_{j}}-z(0)\right|^{2} \leq \int_{-\infty}^{-k} h(s) d s+\left|y_{0}-z(0)\right|^{2}$. Taking the $k \rightarrow \infty$ limit in the last inequality we infer that

$$
\limsup _{j}\left|\varphi\left(t_{m_{j}}, \vartheta_{-t_{m_{j}}} \omega\right) x_{m_{j}}-z(0)\right|^{2} \leq\left|y_{0}-z(0)\right|^{2}
$$

which proves claim (8.5).

Step V. Proof of (8.17). We begin with estimating the first term on the RHS of (8.16). If $-t_{n^{(k)}}<-k$, then by (6.26) and (8.10) we infer that

$$
\begin{gathered}
\left|\varphi\left(t_{n^{(k)}}-k, \vartheta_{-t_{n}(k)} \omega\right) x_{n^{(k)}}-z(-k)\right|^{2} e^{-\nu \lambda_{1} k} \\
=\left|v\left(-k,-t_{n^{(k)}} ; \vartheta_{-k} \omega, x_{n^{(k)}}-z\left(-t_{n^{(k)}}\right)\right)\right|^{2} e^{-\nu \lambda_{1} k} \\
\leq e^{-\nu \lambda_{1} k}\left\{\left|x_{n^{(k)}}-z\left(-t_{\left.n^{(k)}\right)}\right)\right|^{2} e^{-\nu \lambda_{1}\left(t_{n^{(k)}}-k\right)+\frac{3 C^{2}}{\nu} \int_{-t_{n}(k)}^{-k}|z(s)|_{\mathbb{L}^{4}}^{2} d s}\right. \\
\left.\quad \frac{3}{\nu} \int_{-t_{n^{(k)}}}^{-k}\left[|g(s)|_{\mathrm{V}^{\prime}}^{2}+|f|_{\mathrm{V}^{\prime}}^{2}\right] e^{-\nu \lambda_{1}(-k-s)+\frac{3 C^{2}}{\nu} \int_{s}^{-k}\left(|z(\zeta)|_{\mathbb{L}^{4}}^{2}\right) d \zeta} d s\right\} \\
\leq 2 I_{n^{(k)}}+2 I I_{n^{(k)}}+\frac{3}{\nu} I I I_{n^{(k)}}+\frac{3}{\nu}|f|_{\mathrm{V}^{\prime}}^{2} I V_{n^{(k)}},
\end{gathered}
$$


where

$$
\begin{aligned}
& I_{n^{(k)}}=\left|x_{n^{(k)}}\right|^{2} e^{-\nu \lambda_{1} t_{n}(k)+\frac{3 C^{2}}{\nu} \int_{-t}^{-k}{ }_{n(k)}}|z(s)|_{\mathbb{L}^{4}}^{2} d s, \\
& I I_{n^{(k)}}=\left|z\left(-t_{n^{(k)}}\right)\right|^{2} e^{-\nu \lambda_{1} t_{n}(k)+\frac{3 C^{2}}{\nu} \int_{-t_{n}(k)}^{-k}|z(s)|_{\mathbb{L}^{4}}^{2} d s}, \\
& I I I_{n^{(k)}}=\int_{-t_{n}(k)}^{-k}|g(s)|_{\mathrm{V}^{\prime}}^{2} e^{\nu \lambda_{1} s+\frac{3 C^{2}}{\nu} \int_{s}^{-k}|z(\zeta)|_{\mathbb{L}^{4}}^{2} d \zeta} d s, \\
& N_{n^{(k)}}=\int_{-t_{n^{\prime}}}^{-k} e^{\nu \lambda_{1} s+\frac{3 C^{2}}{\nu} \int_{s}^{-k}|z(\zeta)|_{L^{4}}^{2} d \zeta} d s .
\end{aligned}
$$

First we will find a nonnegative function $h \in L^{1}(-\infty, 0)$ such that

$$
\begin{aligned}
& \limsup _{n^{(k)} \rightarrow \infty}\left|\varphi\left(t_{n^{(k)}}-k, \vartheta_{-t_{n}(k)} \omega\right) x_{n^{(k)}}-z(-k)\right|^{2} e^{-\nu \lambda_{1} k} \\
& \leq \int_{-\infty}^{-k} h(s) d s, \quad k \in \mathbb{N} .
\end{aligned}
$$

This will be accomplished as soon as we show the following four results.

We need to prove the following four lemmata.

Lemma 8.4. $\lim \sup I_{k}=0$.

Lemma 8.5. $\limsup _{n^{(k)} \rightarrow \infty} I I_{n^{(k)}}=0$.

Lemma 8.6. $\int_{-\infty}^{0}|g(s)|_{\mathrm{V}^{\prime}}^{2} e^{\nu \lambda_{1} s+\frac{3 C^{2}}{\nu} \int_{s}^{0}|z(\sigma)|_{\mathbb{L}^{4}}^{2} d \sigma} d s<\infty$.

Lemma 8.7. $\int_{-\infty}^{0} e^{\nu \lambda_{1} s+\frac{3 C^{2}}{\nu} \int_{s}^{0}|z(\sigma)|_{\mathbb{L}^{4}}^{2} d \sigma} d s<\infty$.

Proof of Lemma 8.4. Let us recall that $\alpha \in \mathbb{N}, z(t)=z_{\alpha}(t), t \in \mathbb{R}$, being the Ornstein-Uhlenbeck process from subsection 6.3 and one has $\mathbb{E}|z(0)|_{\mathrm{X}}^{2}=\mathbb{E}\left|z_{\alpha}(0)\right|_{\mathrm{X}}^{2}$ $<\frac{\nu^{2} \lambda_{1}}{6 C^{2}}$. Let us recall that the space $\hat{\Omega}(\xi, \mathrm{E})$ was constructed in such a way that

$$
\lim _{n^{(k)} \rightarrow \infty} \frac{1}{-k-\left(-t_{n^{(k)}}\right)} \int_{-t_{n(k)}}^{-k}\left|z_{\alpha}(s)\right|_{\mathrm{X}}^{2} d s=\mathbb{E}|z(0)|_{\mathrm{X}}^{2}<\infty .
$$

Therefore, since the embedding $\mathrm{X} \hookrightarrow \mathbb{L}^{4}(\mathcal{O})$ is a contraction, we have for $n^{(k)}$ sufficiently large,

$$
\frac{3 C^{2}}{\nu} \int_{-t_{n}(k)}^{-k}|z(s)|_{\mathbb{L}^{4}}^{2} d s<\frac{\nu \lambda_{1}}{2}\left(t_{n^{(k)}}-k\right) .
$$

Since the set $B$ is bounded in $\mathrm{H}$, there exists $\rho_{1}>0$ such that for all $n^{(k)},\left|x_{n^{(k)}}\right| \leq$ $\rho_{1}$. Hence,

$$
\begin{array}{r}
\limsup _{n^{(k)} \rightarrow \infty}\left|x_{n^{(k)}}\right|^{2} e^{-\nu \lambda_{1} t_{n}(k)+\frac{3 C^{2}}{\nu} \int_{-t_{n}(k)}^{-k}|z(s)|_{\mathbb{L}^{4}}^{2} d s} \\
\leq \limsup _{n^{(k)} \rightarrow \infty} \rho_{1}^{2} e^{-\frac{\nu \lambda_{1}}{2}\left(t_{n^{(k)}}-k\right)}=0 .
\end{array}
$$


Proof of Lemma 8.7, Denote $p(s)=\nu \lambda_{1} s+\frac{3 C^{2}}{\nu} \int_{s}^{0}|z(s)|_{\mathbb{L}^{4}}^{2}$. We need to show that $\int_{-\infty}^{0} e^{p(s)} d s<\infty$. As in the proof of Lemma 8.4 we have, for $s \leq s_{0}, p(s)<\frac{\nu \lambda_{1}}{2} s$. Hence the result.

Proof of Lemma 8.5. Because of (6.7), we can find $\rho_{2} \geq 0$ and $s_{0}<0$, such that,

$$
\frac{|z(s)|}{|s|}, \frac{|z(s)|_{\mathrm{V}^{\prime}}}{|s|} \quad \text { and } \quad \frac{|z(s)|_{\mathbb{L}^{4}}}{|s|} \leq \rho_{2}, \quad \text { for } s \leq s_{0}
$$

Hence by (8.20) we infer that

$$
\begin{aligned}
& \limsup _{n^{(k)} \rightarrow \infty}\left|z\left(-t_{n^{(k)}}\right)\right|^{2} e^{\int_{-{ }_{n}(k)}^{-k}\left(-\nu \lambda_{1}+\frac{3 C^{2}}{\nu}|z(s)|_{\mathbb{L}^{4}}^{2}\right) d s} \\
& \leq \limsup _{n^{(k)} \rightarrow \infty} \frac{\left|z\left(-t_{n^{(k)}}\right)\right|^{2}}{\left|t_{n^{(k)}}\right|^{2}} \limsup _{n^{(k)} \rightarrow \infty}\left|t_{n^{(k)}}\right|^{2} e^{-\frac{\nu \lambda_{1}\left({ }_{n}(k)-k\right)}{2}} \leq 0 .
\end{aligned}
$$

This finishes the proof of Lemma 8.5 .

Proof of Lemma 8.6. This proof follows the lines of the proof of Lemma 8.7 by taking into account the inequality (8.22). Indeed, since $|g(s)|_{\mathrm{V}^{\prime}}^{2}=|\alpha z(s)+2 B(z(s))|_{\mathrm{V}^{\prime}}^{2}$ $\leq 2 \alpha^{2}|z(s)|_{\mathrm{V}^{\prime}}^{2}+2 C|z(s)|_{\mathbb{L}^{4}}^{4}$, we only need to show that the integrals

$\int_{-\infty}^{0}|z(s)|_{\mathbb{L}^{4}}^{4} e^{\nu \lambda_{1} s+\frac{3 C^{2}}{\nu} \int_{s}^{0}|z(\sigma)|_{\mathbb{L}^{4}}^{2} d \sigma} d s \quad$ and $\quad \int_{-\infty}^{0}|z(s)|_{\mathrm{V}^{\prime}}^{2} e^{\nu \lambda_{1} s+\frac{3 C^{2}}{\nu} \int_{s}^{0}|z(\sigma)|_{\mathbb{L}^{4}}^{2} d \sigma} d s$

are finite.

Therefore, the proof of (8.19) is concluded, and it only remains to finish the proof of (8.17), which we are going to do right now. Let us denote $\tilde{y}_{k}=y_{k}-z(-k)$ and

$$
\begin{aligned}
v^{n^{(k)}}(s) & =v\left(s,-k ; \omega, \varphi\left(t_{n^{(k)}}-k, \vartheta_{-t_{n^{(k)}}} \omega\right) x_{n^{(k)}}-z(-k)\right), s \in(-k, 0), \\
v_{k}(s) & =v\left(s,-k ; \omega, y_{-k}-z(-k)\right), s \in(-k, 0) .
\end{aligned}
$$

From (8.7) and Lemma 7.1 we infer that

$$
v^{n^{(k)}}(\cdot) \rightarrow v_{k} \text { weakly in } L^{2}(-k, 0 ; \mathrm{V}) .
$$

Since $e^{\nu \lambda_{1}} \cdot g(\cdot), e^{\nu \lambda_{1}} \cdot f \in L^{2}\left(-k, 0 ; \mathrm{V}^{\prime}\right)$, we get

$$
\lim _{n^{(k)} \rightarrow \infty} \int_{-k}^{0} e^{\nu \lambda_{1} s}\left\langle g(s), v^{n^{(k)}}(s)\right\rangle d s=\int_{-k}^{0} e^{\nu \lambda_{1} s}\left\langle g(s), v_{k}(s)\right\rangle d s
$$

and

$$
\lim _{n^{(k)} \rightarrow \infty} \int_{-k}^{0} e^{\nu \lambda_{1} s}\left\langle f, v^{n^{(k)}}(s)\right\rangle d s=\int_{-k}^{0} e^{\nu \lambda_{1} s}\left\langle f, v_{k}(s)\right\rangle d s .
$$

On the other hand, using the same methods as those in the proof of Theorem4.5. there exists a subsequence of $\left\{v^{n^{(k)}}\right\}$, which, for the sake of simplicity of notation, is denoted as the old one and which satisfies

$$
v^{n^{(k)}} \rightarrow v_{k} \quad \text { strongly in } L^{2}\left(-k, 0 ; \mathbb{L}_{l o c}^{2}(D)\right) .
$$


Next, since $z(t)$ is an $\mathbb{L}^{4}$-valued process, so is $e^{\nu \lambda_{1} t} z(t)$. Thus by Corollary 5.3 , (8.24) and (8.27), we infer that

$$
\begin{gathered}
\lim _{n^{(k)} \rightarrow \infty} \int_{-k}^{0} e^{\nu \lambda_{1} s} b\left(v^{n^{(k)}}(s), z(s), v^{n^{(k)}}(s)\right) d s \\
=\int_{-k}^{0} e^{\nu \lambda_{1} s} b\left(v_{k}(s), z(s), v_{k}(s)\right) d s .
\end{gathered}
$$

Moreover, since the norms [.] and $\|\cdot\|$ are equivalent on $\mathrm{V}$, and since for any $s \in(-k, 0], e^{-\nu k \lambda_{1}} \leq e^{\nu \lambda_{1} s} \leq 1,\left(\int_{-k}^{0} e^{\nu \lambda_{1} s[\cdot]^{2}} d s\right)^{1 / 2}$ is a norm in $L^{2}(-k, 0 ; \mathrm{V})$ equivalent to the standard one. Hence, from (8.24) we obtain,

$$
\int_{-k}^{0} e^{\nu \lambda_{1} s}\left[v_{k}(s)\right]^{2} d s \leq \liminf _{n^{(k)} \rightarrow \infty} \int_{-k}^{0} e^{\nu \lambda_{1} s}\left[v^{n^{(k)}}(s)\right]^{2} d s .
$$

In other words,

$$
\limsup _{n^{(k)} \rightarrow \infty}\left\{-\int_{-k}^{0}\left[v^{n^{(k)}}(s)\right]^{2} d s\right\} \leq-\int_{-k}^{0} e^{\nu \lambda_{1} s}\left[v_{k}(s)\right]^{2} d s .
$$

From (8.16), (8.19), (8.28) and (8.29) we infer that

$$
\begin{aligned}
\limsup _{n^{(k)} \rightarrow \infty}\left|\varphi\left(t_{n^{(k)}}, \vartheta_{-t_{n^{(k)}}} \omega\right) x_{n^{(k)}}-z(0)\right|^{2} & \\
\leq \int_{-\infty}^{-k} h(s) & d s+2 \int_{-k}^{0} e^{\nu \lambda_{1} s}\left\{\left\langle B\left(v_{k}(s), z(s)\right), v_{k}(s)\right\rangle\right. \\
& \quad\left\langle\left\langle g(s), v_{k}(s)\right\rangle+\left\langle f, v_{k}(s)\right\rangle-\left[v_{k}(s)\right]^{2}\right\} d s .
\end{aligned}
$$

On the other hand, from (8.9) and (8.11), we have

$$
\begin{aligned}
\left|y_{0}-z(0)\right|^{2} & =\left|\varphi\left(k, \vartheta_{-k} \omega\right) y_{k}-z(0)\right|^{2}=\left|v\left(0,-k ; \omega, y_{k}-z(-k)\right)\right|^{2} \\
& =\left|y_{k}-z(-k)\right|^{2} e^{-\nu \lambda_{1} k}+2 \int_{-k}^{0} e^{\nu \lambda_{1} s}\left\{\left\langle g(s), v_{k}(s)\right\rangle\right. \\
& \left.+\left\langle B\left(v_{k}(s), z(s)\right), v_{k}(s)\right\rangle+\left\langle f, v_{k}(s)\right\rangle-\left[v_{k}(s)\right]^{2}\right\} d s
\end{aligned}
$$

Hence, by combining (8.30) with (8.31), we get

$$
\begin{aligned}
& \limsup _{n^{(k)} \rightarrow \infty}\left|\varphi\left(t_{n^{(k)}}, \vartheta_{-t_{n^{(k)}}} \omega\right) x_{n^{(k)}}-z(0)\right|^{2} \\
\leq & \int_{-\infty}^{-k} h(s) d s+\left|y_{0}-z(0)\right|^{2}-\left|y_{k}-z(-k)\right|^{2} e^{-\nu \lambda_{1} k} \\
\leq & \int_{-\infty}^{-k} h(s) d s+\left|y_{0}-z(0)\right|^{2},
\end{aligned}
$$

which proves (8.17), and hence the proof of Proposition 8.1 is finished.

Now we are ready to state and prove the main result of this section.

Theorem 8.8. Suppose that a domain $D \subset \mathbb{R}^{2}$ is such that the Poincaré inequality (4.2) holds on it. Consider the metric $D S \mathfrak{T}=(\hat{\Omega}(\xi, \mathrm{E}), \hat{\mathcal{F}}, \hat{\mathbb{P}}, \hat{\vartheta})$ (see Proposition 6.13), and consider the $R D S \varphi$ over $\mathfrak{T}$ generated by the $2 \mathrm{D}$ stochastic Navier-Stokes equations (4.16) with additive noise such that Assumption $\mathrm{A.1}$ is satisfied. Then the $R D S \varphi$ is asymptotically compact. 
Proof. Let $B \subset \mathrm{H}$ be a bounded set. In view of Proposition 8.1 it is sufficient to prove that there exists a closed bounded random set $K(\omega) \subset \mathrm{H}$ which absorbs $B$. In fact, we will show below that an even stronger property holds. Namely, that there exists a closed bounded random set $K(\omega) \subset \mathrm{H}$ which absorbs every bounded deterministic set $B \subset \mathrm{H}$.

Let $\omega \in \Omega$ be fixed. For given $s \leq 0$ and $x \in \mathrm{H}$, let $v$ be the solution of (4.19) on $[s, \infty)$ with the initial condition $v(s)=x-z(s)$. Applying (8.10) with $t=0, \tau=s \leq 0$, we get

$$
\begin{aligned}
|v(0)|^{2} \leq & 2|x|^{2} e^{\nu \lambda_{1} s+\frac{3 C^{2}}{\nu} \int_{s}^{0}|z(r)|_{\mathrm{L}^{4}}^{2} d r}+2|z(s)|^{2} e^{\nu \lambda_{1} s+\frac{3 C^{2}}{\nu} \int_{s}^{0}|z(r)|_{\mathrm{L}^{4}}^{2} d r} \\
& +\frac{3}{\nu} \int_{s}^{0}\left\{|g(t)|_{\mathrm{V}^{\prime}}^{2}+|f|^{2}\right\} e^{\nu \lambda_{1} t+\frac{3 C^{2}}{\nu} \int_{t}^{0}|z(r)|_{\mathrm{L}^{4}}^{2} d r} d t .
\end{aligned}
$$

Set

$$
\begin{aligned}
r_{1}(\omega)^{2}=2 & +\sup _{s \leq 0}\left\{2|z(s)|^{2} e^{\nu \lambda_{1} s+\frac{3 C^{2}}{\nu} \int_{s}^{0}|z(r)|_{\mathbb{L}^{4}}^{2} d r}\right\} \\
& +\frac{3}{\nu} \int_{-\infty}^{0}\left\{|g(t)|_{\mathrm{V}^{\prime}}^{2}+|f|^{2}\right\} e^{\nu \lambda_{1} t+\frac{3 C^{2}}{\nu} \int_{t}^{0}|z(r)|_{\mathbb{L}^{4}}^{2} d r} d t .
\end{aligned}
$$

Similarly to (8.23) we can prove that

$$
\limsup _{t_{0} \rightarrow-\infty}\left|z\left(t_{0}\right)\right|^{2} e^{\nu \lambda_{1} t_{0}+\frac{3 C^{2}}{\nu} \int_{t_{0}}^{0}|z(r)|_{\mathbb{L}^{4}}^{2} d r}=0 .
$$

Hence, by the continuity of the map $s \mapsto z(s) \in \mathrm{H}$,

$$
\sup _{s \leq 0}|z(s)|^{2} e^{\nu \lambda_{1} s+\frac{3 C^{2}}{\nu} \int_{s}^{0}|z(r)|_{\mathbb{L}^{4}}^{2} d r}<\infty
$$

and hence in conjunction with Lemmata 8.6 and 8.7, we infer that

$$
r_{1}^{2}(\omega)<\infty, \quad \text { for all } \omega \in \Omega \text {. }
$$

On the other hand, given $\rho>0$, by (8.21) we can find $t_{\rho}(\omega) \leq 0$ such that, for all $s \leq t_{\rho}(\omega), \rho^{2} e^{\nu \lambda_{1} s+\frac{3 C^{2}}{\nu} \int_{s}^{0}|z(r)|_{\mathbb{L}^{4}}^{2} d r} \leq 1$. Therefore, from (8.32), if $|x| \leq \rho$ and $s \leq t_{\rho}(\omega),|v(0, \omega ; s, x-z(s))|^{2} \leq r_{1}^{2}(\omega)$. Thus, we infer that

$$
|u(0, s ; \omega, x)| \leq|v(0, s ; \omega, x-z(s))|+|z(0)| \leq r_{2}(\omega), \quad \text { for all } \omega \in \Omega,
$$

where $r_{2}(\omega)=r_{1}(\omega)+|z(0, \omega)|$. From (8.33) and our assumptions, we infer that for all $\omega \in \Omega, r_{2}(\omega)<\infty$. Defining $K(\omega):=\left\{u \in \mathrm{H}:|u| \leq r_{2}(\omega)\right\}$ concludes the proof.

Remark 8.9. Here, we would like to point out that although we have proved the Asymptotic Compactness of the Random Dynamic System generated by the 2D stochastic NSes, we still cannot obtain the existence of the attractor for this RDS. The reason is that although from the proofs of Proposition 8.1 and Theorem 8.8 we can construct a bounded closed random set $K(\omega)$ in $H$ (even in $V$; see [9]), which attracts all bounded deterministic sets, due to lack of the Sobolev compact embedding, we cannot directly obtain the compactness of $K(\omega)$ neither in $V$ nor $H$. In this case, when $K(\omega)$ is bounded, we obtain only the dissipativity of our RDS. The dissipativity plus the asymptotic compactness properties are not enough to deduce the compactness of $K(\omega)$. Hence, we will need further properties of our RDS and leave the existence of abstractors for the RDS as an open question. 


\section{INVARIANT MEASURES}

In this very short section we only state a result which is a direct consequence of Corollary 4.4 in [18] and of our Theorems 3.4 and 8.8 ,

Let $u(t, x)$ be the unique solution of problem (4.16). Let us recall that such a unique solution exists for each $x \in \mathrm{H}$. We define the transition operator $P_{t}$ by a standard formula. For $g \in \mathcal{C}_{b}(\mathrm{H})$, we put

$$
P_{t} g(x)=\mathbb{E}[g(u(t, x))], \quad x \in \mathrm{H} .
$$

In view of Proposition $3.8 P_{t}, t \geq 0$, is a family of Feller operators, i.e. $P_{t}$ : $C_{b}(\mathrm{H}) \rightarrow C_{b}(\mathrm{H})$ and, for any $g \in \mathcal{C}_{b}(\mathrm{H})$ and $x \in \mathrm{H}, P_{t} g(x) \rightarrow g(x)$ as $t \searrow 0$. Moreover, as in [18] one can prove that $\varphi$ is a Markov RDS, i.e. $P_{t+s}=P_{t} P_{s}$ for all $t, s \geq 0$. Hence from Corollary 3.7 we now have the following result.

Corollary 9.1. There exists an invariant measure for the Stochastic NSEs (4.16).

Remark 9.2. It is an interesting question whether the method of [38 can be extended so that not only the estimates in the mean but also pathwise can be proved. A positive answer to this question would prove that the Random Dynamical System generated by stochastic Ginzburg-Landau equations is asymptotically compact and hence could be an indication of the existence of a global attractor.

\section{ACKNOWLEDGEMENTS}

The authors would like to thank David Elworthy and Hans Crauel for their interest in this work. Special thanks to Benedetta Ferrario, Jan van Neerven and Dona Strauss for careful reading of the manuscript and pointing out several mistakes. Preliminary versions of this work were presented at various conferences: the first author spoke in Warwick (July 2001) and Trento (January 2002), and the second author, Warwick (July 2002) and Beijing (August 2002). The authors would like to thank the organizers of those meetings, in particular David Elworthy, Roger Tribe, Sergio Albeverio, Giuseppe Da Prato, Michael Röckner, Luciano Tubaro and Ma Zhi-Ming for their kind invitations. We would like to thank an anonymous referee for careful reading of the manuscript and useful remarks.

\section{REFERENCES}

[1] F. Abergel, Existence and finite dimensionality of the global attractor for evolution equations on unbounded domains, Journal of Differential Equations 83(1), 85-108 (1990). MR.1031379 (90m:58121)

[2] L. Arnold, Random dynamical systems, Springer-Verlag, Berlin, Heidelberg, New York, 1998. MR:1723992 (2000m:37087)

[3] P. Baxendale, Gaussian measures on Function Spaces, Amer. J. Math. 98, 891-952 (1976). MR0467809 (57:7660)

[4] Z. Brzeźniak, On analytic dependence of solutions of Navier-Stokes equations with respect to exterior force and initial velocity, Universitatis Iagellonicae Acta Mathematica, Fasciculus XXVIII, 111-124 (1991). MR1136785 (92m:35202)

[5] Z. Brzeźniak, On Sobolev and Besov spaces regularity of Brownian paths, Stochastics Stochastics Rep. 56, no. 1-2, 1-15 (1996). MR 1396751 (97g:60104)

[6] Z. Brzeźniak, Stochastic Convolution in Banach spaces, Stochastics and Stochastics Reports 61, 245-295 (1997). MR 1488138

[7] Z. Brzeźniak, Some remarks on Itô and Stratonovich integration in 2-smooth Banach spaces, pp. 48-69 in Proceedings of the Swansea 2002 Workshop on Probabilistic Methods in Fluids, World Scientific, New Jersey, London, Singapore, Hong Kong, 2003; eds.: I.M. Davies, N. Jacob, A. Truman, O. Hassan, K. Morgan, N.P. Weatherill. MR2083364 (2005g:60085) 
[8] Z. Brzeźniak, M. Capiński and F. Flandoli, Pathwise global attractors for stationary random dynamical systems, Probability Theory and Related Fields 95, 87-102 (1993). MR 1207308 (94b:60067)

[9] Z. Brzeźniak and Y. Li, Asymptotic behaviour of solutions to the 2D stochastic Navier-Stokes equations in unbounded domains - new developments, Proceedings of the First Sino-German Conference in Stochastic Analysis, 28 August-3 September, 2002, Beijing, in press.

[10] Z. Brzeźniak and S. Peszat, Maximal Inequalities and Exponential Estimates for Stochastic Convolutions in Banach Spaces, Gesztesy, Fritz (ed.) et al., Stochastic Processes, Physics and Geometry: New interplays. I. A volume in honor of Sergio Albeverio. Proceedings of the conference on infinite dimensional (stochastic) analysis and quantum physics, Leipzig, Germany, January 18-22, 1999, Providence, RI: American Mathematical Society (AMS). CMS Conf. Proc. 28, 55-64 (2000). MR1803378 (2001k:60084)

[11] Z. Brzeźniak and S. Peszat, Stochastic two dimensional Euler equations, Ann. Probab. 29, no. 4, 1796-1832 (2001). MR.1880243 (2002m:60091)

[12] Z. Brzeźniak and J. van Neerven, Space-time regularity for linear stochastic evolution equations driven by spatially homogeneous noise. J. Math. Kyoto Univ. 43, no. 2, 261-303 (2003). MR2051026 (2005c:60077)

[13] H. Cartan, Differential Calculus, Hermann, Paris, 1971. MR0344032 (49:8772)

[14] L. Cattabriga, Su un problema al contorno relativo al sistema di equazioni di Stokes, Rend. Sem. Mat. Univ. Padova 31, 308-340 (1961). MR0138894 (25:2334)

[15] C. Castaing and M. Valadier, Convex Analysis and Measurable Multifunctions, Lecture Notes in Mathematics 580, Springer, Berlin, 1977. MR0467310 (57:7169)

[16] H. Crauel, Random Probability Measures on Polish Spaces, Habilitationsschrift, Bremen, 1995; Stochastics Monographs, vol. 11, Taylor \& Francis, London, 2002. MR1993844 (2004e:60005)

[17] H. Crauel, A. Debussche and F. Flandoli, Random attractor, Journal of Dynamics and Differential Equations 9(2), 307-341 (1997). MR1451294 (98c:60066)

[18] H. Crauel and F. Flandoli, Attractors for random dynamical systems, Probability Theory and Related Fields 100, 365-393 (1994). MR.1305587 (95k:58092)

[19] G. Da Prato and A. Debussche, Two-dimensional Navier-Stokes equations driven by a spacetime white noise, J. Funct. Anal. 196, no. 1, 180-210 (2002). MR.1941997 (2003h:35198)

[20] G. Da Prato and J. Zabczyk, Stochastic EQuations in infinite Dimensions, Encyclopedia of Mathematics and its Applications 44 Cambridge University Press, Cambridge, 1992. MR.1207136 (95g:60073)

[21] G. Da Prato and J. Zabczyk, Ergodicity for Infinite Dimensional Systems, London Mathematical Society Lecture Note Series 229, Cambridge University Press, Cambridge, 1996. MR1417491 (97k:60165)

[22] J.-P. Eckmann and M. Hairer, Invariant measures for stochastic partial differential equations in unbounded domains, Nonlinearity 14, no. 1, 133-151 (2001). MR1808628 (2002a:60103)

[23] F. Flandoli, Dissipativity and invariant measures for stochastic Navier-Stokes equations with a generalised noise, NoDEA 1, 403-423 (1994). MR1300150 (95h:35254)

[24] F. Flandoli and V.M. Tortorelli, Time discretization of Ornstein-Uhlenbeck equations and stochastic Navier-Stokes equations with a generalised noise, Stochastics Stochastics Rep. 55, no. 1-2, 141-165 (1995). MR 1382289 (97a:35270)

[25] A. Friedman, Partial differential equations, Holt, Rinehart and Winston, Inc., 1969. MR0445088 (56:3433)

[26] D. Fujiwara, and H. Morimoto, An $L_{r}$ theorem of the Helmhotz decomposition of vector fields, J. Fac. Sci. Univ. Tokyo Sect. IA Math. 24, 685-700 (1977). MR0492980 (58:12023)

[27] J.M. Ghidaglia, A note on the strong convergence towards attractors of damped forced KdV equations, J. Differential Equations 110, no. 2, 356-359 (1994). MR1278375 (95d:35150)

[28] L. Gross, Measurable functions on Hilbert space, Trans. Am. Math. Soc. 105, 372-390 (1962). MR0147606 (26:5121)

[29] M. Hairer, Ergodicity of stochastic differential equations driven by fractional Brownian motion, Ann. Probab. 33 703-758 (2005). MR2123208(2005k:60178)

[30] J. G. Heywood, The Navier-Stokes equations: on the existence, regularity and decay of solutions, Indiana Univ. Math. J. 29, no. 5, 639-681 (1980). MR0589434 (81k:35131)

[31] S.G. Jones, Stability and asymptotic fixed-point theory, Proc. Nat. Acad. Sci. U.S.A. 53, 1262-1264 (1965). MR0180728 (31:4959) 
[32] H. Keller and B. Schmalfuss, Attractors For Stochastic Sine Gordon Equations Via Transformation into Random Equations, preprint, the University of Bremen, 1999.

[33] O. Ladyzhenskaya, Attractors For Semigroups And evolution Equations, Lezioni Lincee, Cambridge University Press, Cambridge, 1991. MR.1133627 (92k:58040)

[34] J. L. Lions and E. Magenes, Non-Homogeneous Boundary Value Problems and AppliCAtions, vol. 1, Springer-Verlag, Berlin, Heidelberg, New York, 1972. MR0350177 (50:2670)

[35] J.L. Lions and G. Prodi, Un théorème d'existence et unicité dans les équations de NavierStokes en dimension 2, C. R. Acad. Sci. Paris 248, 3519-3521 (1959). MR0108964 (21:7676)

[36] A.L. Neidhardt, Stochastic Integrals in 2-uniformly smooth Banach Spaces, University of Wisconsin, 1978.

[37] R. Rosa, The global attractor for the 2D Navier-Stokes flow on some unbounded domains, Nonlinear Analysis 32, 71-85 (1998). MR.1491614 (98k:35152)

[38] J. Rougemont, Space-time invariant measures, entropy, and dimension for stochastic Ginzburg-Landau equations, Comm. Math. Phys. 225, no. 2, 423-448 (2002). MR1889231 (2002m:37073)

[39] B. Schmalfuss, Backward cocycles and attractors of Stochastic Differential Equations", pp. 185-192 in International Seminar on Applied Mathematics-Nonlinear Dynamics: Attractor Approximation and Global Behaviour, eds. Reitmann, T. Riedrich and N. Koksch, 1992.

[40] R. Temam, Navier-Stokes Equations, North-Holland Publish Company, Amsterdam, 1979. MR0603444 (82b:35133)

[41] R. Temam, Infinite-Dimensional Dynamical Systems in Mechanics and Physics, Second Edition, Springer, New York, 1997. MR1441312 (98b:58056)

[42] J. Toth and S. Zelditch, $L^{p}$ norms of eigenfunctions in the completely integrable case. Ann. Henri Poincaré 4, no. 2, 343-368 (2003). MR1985776 (2004g:58043)

Department of Mathematics, The University of Hull, Hull, HU6 7RX, United KingDOM

Current address: Department of Mathematics, University of York, Heslington, York, YO10 5DD, United Kingdom

E-mail address: zb500@york.ac.uk

Department of Mathematics, The University of Hull, Hull, Hu6 7RX, United KingDOM

Current address: School of Hydropower and Information Engineering, Huazhong University of Science and Technology, Wuhan 430074, People's Republic of China

E-mail address: chuchuemma@163.com 